\title{
Produtividade
}

\section{da compacidade enumerável em grupos topológicos}

\author{
Danilo Dias da Silva \\ DISSERTAÇÃO APRESENTADA \\ $\mathrm{AO}$ \\ INSTITUTO DE MATEMÁTICA E ESTATÍSTICA \\ DA \\ UNIVERSIDADE DE SÃO PAULO \\ PARA \\ OBTENÇÃO DO TÍTULO DE MESTRE \\ $\mathrm{EM}$ \\ CIÊNCIAS \\ Programa: Matemática \\ Orientador: Prof. Dr. Artur Hideyuki Tomita
}

Durante a elaboração deste trabalho, o autor recebeu apoio financeiro do CNPq.

- São Paulo, 30 de junho de 2009 - 


\title{
Produtividade da compacidade enumerável em grupos topológicos
}

\author{
Este exemplar corresponde à redação \\ final da dissertação devidamente corrigida \\ e defendida por Danilo Dias da Silva e \\ aprovada pela comissão julgadora.
}

São Paulo, 21 de julho de 2009.

\section{Banca examinadora:}

Prof. Dr. Artur Hideyuki Tomita (orientador)

IME-USP

Profa. Dra. Ofelia Teresa Alas

IME-USP

Profa. Dra. Irene Castro

UFPA 
Dedico esta dissertação aos meus pais. 


\section{Agradecimentos}

Primeiramente, agradeço aos professores do IME-USP pela dedicação e pelo profissionalismo. Além do conhecimento matemático transmitido a mim durante os dois anos do mestrado, valorizo o exemplo de profissionais que levam o ensino público a sério, o que reforça minhas convicções.

Agradeço aos alunos de pós-graduação pelo ambiente de companheirismo que encontrei quando cheguei ao IME. Este ambiente foi determinante para a minha escolha de permanecer em São Paulo e fez com que a minha rotina de estudos fosse bem mais leve e divertida.

Agradeço aos meus amigos de Aracaju que dividiram comigo a experiência de morar em São Paulo nestes dois anos e que amenizaram a falta de casa. Infelizmente não poderei citar todos por serem muitos, mas agradeço em especial a Marco e Débora pelo interesse que demonstraram na minha vinda e por toda ajuda que deram para que isto se concretizasse.

Agradeço ao meu orientador Artur, a quem posso chamar de amigo. Obrigado por toda a boa vontade que sempre demonstrou ao me ensinar, pelas conversas e por toda a ajuda durante o mestrado.

Agradeço especialmente aos meus pais e irmãs. Palavras não são suficientes.

Agradeço, por fim, ao CNPq pelo apoio financeiro. 


\section{Resumo}

O principal objetivo desta dissertação é estudar a produtividade da compacidade enumerável em grupos topológicos. Vários contra-exemplos foram descritos. Em 1980, E. van Douwen construiu com a ajuda do Axioma de Martin dois grupos topológicos enumeravelmente compactos cujo produto não era enumeravelmente compacto [2]. Em 1991, Hart e van Mill construiram com a ajuda de uma versão enumerável do Axioma de Martin um grupo enumeravelmente compacto, mas cujo quadrado não era [6]. Nesta dissertação, pode-se encontrar os dois exemplos. O conhecimento sobre a não-produtividade da compacidade enumerável foi bastante ampliado recentemente em trabalhos publicados por Tomita [9], [10] e [11]. Exemplos de não-produtividade para o caso quadrado foram encontrados baseados na existência de ultrafiltros seletivos sem o uso do Axioma de Martin [5] e, mais recentemente, exemplos ainda mais elaborados foram obtidos: dado um $k \in \omega$, existe um grupo enumeravelmente compacto $G$ tal que $G^{k}$ é enumeravelmente compacto, mas $G^{k+1}$ não é [10]; existe um grupo enumeravelmente compacto $G$ tal que $G^{\alpha}$ é enumeravelmente compacto para todo $\alpha<2^{\mathfrak{c}}$, mas $G^{2^{\mathfrak{c}}}$ não é [9]; e finalmente foi obtido um grupo $G$ tal que, dado $\alpha<2^{\mathfrak{c}}, G^{\gamma}$ é enumeravelmente compacto para todo $\gamma \leq \alpha$, mas $G^{\alpha}$ não o é [9]. Todos estes contra-exemplos podem ser encontrados nesta dissertação. 


\section{Abstract}

The main purpose of this work is to study the property of countable compactness in topological groups regarding its productivity. We shall present many counter-examples. In 1980, E. van Douwen produced two countably compact groups whose product is not countably compact from MA [2]. In 1991, Hart e van Mill showed that there exists a countably compact topological group whose square is not countably compact under $\mathrm{MA}_{\text {countable }}[6]$. One can find both constructions in this work. The knowledge about the nonproductivity of countable compactness in topological groups has been increased in published papers by Tomita [9], [10] e [11]. Examples of non-productivity of countably compactness regarding the square of topological groups were found based on selective ultrafilters without the use of MA [5] and, more recently, even more intricated examples were found: given $k \in \omega$, there exists a countably compact group $G$ such that $G^{k}$ is countably compact, but $G^{k+1}$ is not [10]; there exists a countably compact group $G$ such that $G^{\alpha}$ is countably compact for all $\alpha<2^{\mathfrak{c}}$, but $G^{2^{\mathfrak{c}}}$ is not [9]; and finally there exists a countably compact group $G$ such that, given $\alpha<2^{\mathfrak{c}}, G^{\gamma}$ is countably compact for all $\gamma \leq \alpha$, but $G^{\alpha}$ is not [9]. All these counter-examples can be found in this work. 


\section{Sumário}

$\begin{array}{ll}\text { Introdução } & 1\end{array}$

1 Notações, definições e resultados preliminares $\quad 4$

1.1 Grupos topológicos enumeravelmente compactos . . . . . . . . . . . . 5

1.2 Ultrafiltros seletivos . . . . . . . . . . . . . . . . . . 12

1.3 Ultraprodutos . . . . . . . . . . . . . . . . . . 13

1.4 Famílias independentes e subgrupos $\omega$-limitados. . . . . . . . . . . . . 15

1.5 Axioma de Martin para ordens parciais enumeráveis $\left(\mathrm{MA}_{\mathrm{e}}\right)$. . . . . . . . 17

2 Primeiros contra-exemplos para a produtividade da compacidade $\begin{array}{ll}\text { enumerável. } & 19\end{array}$

2.1 Exemplo de van Douwen . . . . . . . . . . . . . . . . . . 19

2.2 Primeiro exemplo de Hart e van Mill . . . . . . . . . . . . . . . . . . . . . . 21

2.3 Segundo exemplo de Hart e van Mill . . . . . . . . . . . . . . . . . . . 26

3 Grupo enumeravelmente compacto cujo quadrado não é a partir de um grupo de van Douwen. $\quad 35$

3.1 Preliminares . . . . . . . . . . . . . . . . . . 35

3.2 Construção do grupo de van Douwen . . . . . . . . . . . . . . . . . . . 40 
3.3 Grupo enumeravelmente compacto cujo quadrado não é enumeravelmente compacto a partir de um grupo de van Douwen de ordem p. . . . . . . . . . 45

3.4 Grupo enumeravelmente compacto cujo quadrado não é enumeravelmente compacto a partir de um grupo de van Douwen livre. . . . . . . . . . . . . 53

3.5 Grupo enumeravelmente compacto cujo quadrado não é enumeravelmente compacto a partir de um ultrafiltro seletivo. . . . . . . . . . . . . . 56

4 Produto finito de grupos enumeravelmente compactos.

5 Produto infinito de grupos enumeravelmente compactos. 68

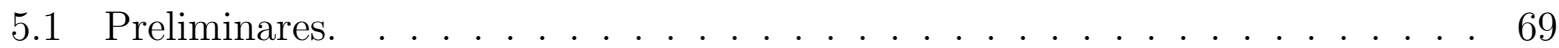

$5.2 H^{\gamma}$ é enumeravelmente compacto para todo $\gamma<2^{\mathfrak{c}}$, mas $H^{2^{\mathfrak{c}}}$ não é. $\quad . \quad . . .73$

$5.3 H^{\gamma}$ é enumeravelmente compacto para todo $\gamma<\alpha$, mas $H^{\alpha}$ não é $\left(\omega \leq \alpha<2^{\mathfrak{c}}\right) .77$

6 Considerações finais 


\section{Introdução}

O estudo da não-produtividade da compacidade enumerável em espaços topológicos começou na década de 1950, quando Terasaka e Novák mostraram que existe um espaço enumeravelmente compacto cujo quadrado não é pseudocompacto. Os casos finito e enumerável foram estudados por Frolík na década de 1960 e o caso não-enumerável por Saks na década de 1970.

Scarbourough e Stone mostraram em 1966 que se $\left\{X_{i}: i \in I\right\}$ é uma família de espaços topológicos, então $\prod_{i \in I} X_{i}$ é enumeravelmente compacto se, e somente se, $\prod_{j \in J} X_{j}$ é enumeravelmente compacto para todo $J \subseteq I$ com $|J| \leq 2^{2^{c}}$.

Ginsburg e Saks melhoraram o teorema acima em 1975. Eles mostraram que $\prod_{i \in I} X_{i}$ é enumeravelmente compacto se, e somente se, $\prod_{j \in J} X_{j}$ é enumeravelmente compacto para todo $J \subseteq I$ com $|J| \leq 2^{\mathfrak{c}}$. Uma questão natural seria se $2^{\mathfrak{c}}$ poderia ser substituído por um cardinal menor.

Saks mostrou em 1979 que $2^{\mathfrak{c}}$ era consistentemente o melhor possível. Ele mostrou que se existem $2^{\mathfrak{c}}$ ultrafiltros seletivos, então existe um espaço enumeravelmente compacto $X$ tal que $X^{\alpha}$ é enumeravelmente compacto para todo $\alpha<2^{\mathfrak{c}}$, mas $X^{2^{\mathfrak{c}}}$ não é.

Comfort e Ross mostraram em 1966 que o produto de grupos pseudocompactos é pseudocompacto. Isto motivou Comfort a perguntar se a compacidade enumerável é produtiva na classe dos grupos topológicos. 
O objetivo deste trabalho é apresentar os principais contra-exemplos que foram encontrados para a produtividade da compacidade enumerável em grupos topológicos desde a questão de Comfort.

E. van Douwen respondeu negativamente essa questão no ano de 1980. Ele construiu dois grupos topológicos enumeravelmente compactos cujo produto não era a partir do Axioma de Martin. Essa construção é dividida em duas partes:

(1) (MA) Existe um grupo infinito enumeravelmente compacto sem sequências nãotriviais convergentes (grupo de van Douwen) como subgrupo do grupo compacto $2^{\mathfrak{c}}$.

(2) (ZFC) Um grupo enumeravelmente compacto de ordem 2 sem sequências nãotriviais convergentes contém dois subgrupos enumeravelmente compactos cujo produto não é enumeravelmente compacto.

Em 1991, também usando o Axioma de Martin Hart e van Mill construiram um grupo topológico enumeravelmente compacto, mas cujo quadrado não era. Este exemplo pode ser encontrado no capítulo 2 desta dissertação. Em 2004, Tomita, Garcia-Ferreira e Watson construiram um grupo de van Douwen a partir de um ultrafiltro seletivo [5]. Em 2005, Tomita obteve a partir de um grupo de van Douwen, um exemplo de grupo enumeravelmente compacto cujo quadrado não era [11]. A construção do grupo de van Douwen e o exemplo podem ser encontrados no capítulo 3. No mesmo ano, usando ultraprodutos, Tomita obteve um grupo enumeravelmente compacto $G$ tal que $G^{k}$ é enumeravelmente compacto, mas $G^{k+1}$ não é. Este resultado foi descrito nesta dissertação com uma demonstração um pouco diferente das encontradas em [10] e [9]. Na secção 5.2 do capítulo 5 foi construído um grupo enumeravelmente compacto $G$ tal que $G^{\alpha}$ é enumeravelmente compacto para todo $\alpha<2^{\mathfrak{c}}$, mas $G^{2^{\mathfrak{c}}}$ não é; e finalmente na secção 5.3 foi obtido um grupo $G$ tal que, dado $\alpha<2^{\mathfrak{c}}, G^{\gamma}$ é enumeravelmente compacto para todo $\gamma \leq \alpha$, mas $G^{\alpha}$ não o é. Estes dois últimos exemplos foram obtidos neste trabalho com demonstrações diferentes das encontradas em [9].

As demontrações dos capítulos 4 e 5 comentadas acima foram obtidas a partir de idéias 
do meu orientador Artur Hideyuki Tomita e foram redigidas pelo autor desta dissertação. 


\section{Capítulo 1}

\section{Notações, definições e resultados}

\section{preliminares}

Neste capítulo, descreveremos as notações, definições e os resultados preliminares que serão utilizados por todo o texto. Notações mais específicas e particulares de um determinado capítulo serão discutidas no início do próprio capítulo para a comodidade do leitor. Estamos assumindo familiariedade do leitor com os conceitos básicos de Topologia Geral como os axiomas de separação, teoria básica de filtros e ultrafiltros e resultados básicos envolvendo compacidade e compacidade enumerável [3] e [12]. Da Teoria dos Conjuntos é assumida familiariedade com a teoria básica de ordinais e cardinais envolvendo conceitos como cofinalidade, cardinais regulares e singulares [7]. Também assumimos conhecimento do

leitor sobre a teoria básica da Álgebra Linear e sobre a teoria básica de Grupos Algébricos, como o grupo quociente e o primeiro teorema do isomorfismo [4]. 
DEFINIÇÕES E RESULTADOS PRELIMINARES

\subsection{Grupos topológicos enumeravelmente compactos}

Começamos por definir os principais conceitos usados nesta dissertação referentes a grupos topológicos enumeravelmente compactos. A maioria das definições e demonstrações podem ser encontradas em qualquer livro de topologia geral como, por exemplo, em [3] e [12]. Reproduzimos aqui alguns desses conceitos com o objetivo de fixá-los na mente do leitor. Os resultados que puderem ser encontrados facilmente em [3] e [12] não serão demonstrados.

Indicaremos por $\mathcal{P}(X)$ a coleção de todos os subconjuntos de um conjunto $X$. A união, intersecção e diferença de dois conjuntos serão denotadas, respectivamente, por $A \cup B, A \cap B$ e $A \backslash B$. Definimos então $A \triangle B=A \backslash B \cup B \backslash A$.

Se $X$ é um conjunto e $\lambda$ é um cardinal, definimos $[X]^{\lambda}=\{A \in \mathcal{P}(X):|A|=\lambda\}$, $[X]^{<\lambda}=\{A \in \mathcal{P}(X):|A|<\lambda\}$ e $[X]^{\leq \lambda}=\{A \in \mathcal{P}(X):|A| \leq \lambda\}$.

Definição 1.1.1: Um espaço topológico $X$ é enumeravelmente compacto se para toda cobertura enumerável formada por conjuntos abertos do espaço $X$ existe uma subcobertura finita de $X$.

Analogamente ao caso compacto, a definição de compacidade enumerável é equivalente a afirmação de que toda família enumerável de fechados que possui a propriedade da intersecção finita tem intersecção não-vazia.

Lema 1.1.1: Um espaço topológico $X$ é enumeravelmente compacto se, e somente se, toda sequência em $X$ possui ponto de acumulação.

Definição 1.1.2: Dado um ultrafiltro livre $p \in \omega^{*}$ e um espaço topológico $X$, dizemos 
que $x \in X$ é o $p$-limite de $\left\{x_{n}: n \in \omega\right\} \subseteq X$ se para toda vizinhança $U$ de $x$ o conjunto $\left\{n \in \omega: x_{n} \in U\right\}$ é um elemento de $p$.

Lema 1.1.2: Um espaço topológico $X$ é enumeravelmente compacto se, e somente se, toda sequência em $X$ possui p-limite para algum $p \in \omega^{*}$.

Demonstração: Suponha que toda sequência em $X$ possui p-limite para algum $p \in \omega^{*}$ e considere $A \subseteq X$ infinito. Existe $\left\{x_{n}: n \in A\right\} \subseteq A \operatorname{com} x_{m} \neq x_{n}$, se $n \neq m$. Por hipótese, existe $x \in X$ tal que $x=p-\lim \left\{x_{n}: n \in \omega\right\}$. Se $U$ é vizinhança aberta de $x$, então $\left\{n \in \omega: x_{n} \in U\right\} \in p$; logo, é infinito. Temos então que $A \cap U$ é infinito e $x$ é ponto de acumulação de $A$. Por outro lado, seja $\left\{x_{n}: n \in \omega\right\}$ sequência em $X$. Se existe $a \in X$ tal que $x_{n}=a, \forall n \in A$ conjunto infinito de $\omega$, então tome $p$ ultrafiltro contendo $\{A\} \cup$ $\cup\{\omega \backslash F: \backslash F$ é finito $\}$. Assim, $p-\lim \left\{x_{n}: n \in \omega\right\}=a$. Seja $B \subseteq \omega$ infinito tal que $x_{m} \neq x_{n}$, se $n, m \in B, n \neq m$. Temos que $\left\{\overline{\left\{x_{n}: n \in B \backslash F\right\}}: F \subseteq B\right.$ finito $\}$ é família enumerável de fechados que atende a propriedade da intersecção finita. Assim, $\bigcap_{F \in[B]<\omega} \overline{\left\{x_{n}: n \in B \backslash F\right\}} \neq \emptyset$. Seja $x \in \bigcap_{F \in[B]<\omega} \overline{\left\{x_{n}: n \in B \backslash F\right\}}$. Para toda vizinhança aberta $V$ de $x$ e para todo $F \in[B]^{<\omega}$ tem-se $V \cap\left\{x_{n}: n \in B \backslash F\right\} \neq \emptyset$, o que implica em $\left\{n \in B \backslash F: x_{n} \in V\right\} \neq \emptyset$. Temos que $\left\{\left\{n \in B \backslash F: x_{n} \in V\right\}: F \in[B]^{<\omega}\right.$ e $V$ vizinhança aberta de $x$ \} atende a propriedade da intersecção finita. Logo, existe ultrafiltro $p$ que contém a família acima e, portanto, $x=p-\lim \left\{x_{n}: n \in \omega\right\}$.

Definição 1.1.3: Um espaço topológico $X$ é p-compacto se toda sequência em $X$ tem p-limite.

Definição 1.1.4: Um grupo é um conjunto $G$ munido de uma operação binária $\cdot: G \times G \longmapsto G$ tal que:

i) $(x \cdot y) \cdot z=x \cdot(y \cdot z), \forall x, y, z \in G$;

ii) $\exists e \in G$ (elemento indentidade) tal que $x \cdot e=e \cdot x=x, \forall x \in G$; 
ii) $\forall x \in G$, existe um único $x^{-1}$ tal que $x \cdot x^{-1}=x^{-1} \cdot x=e$.

Nesta dissertação denotaremos frequentemente $x \cdot y$ por $x y$. Dizemos que o grupo $G$ é abeliano quando $x y=y x, \forall x, y \in G$. Neste caso, podemos denotar $x \cdot y$ também por $x+y$ e o elemento indentidade do grupo por 0. Como todos os grupos topológicos que aparecem nesta dissertação são abelianos, frequentemente usaremos + como sinal para a operação binária de grupo.

Definição 1.1.5: Dizemos que o grupo abeliano $G$ é abeliano livre quando este possui uma base, ou seja, quando possui um subconjunto gerador que é independente. Neste caso, consideramos que $\left\{x_{\xi}: \xi \in \kappa\right\}$ é independente quando dado $F \in[\kappa]^{<\omega}$ qualquer e $\left\{n_{\xi}: \xi \in F\right\} \subseteq \mathbb{Z}$, temos que $\sum_{\xi \in F} n_{\xi} x_{\xi}=0 \Longrightarrow n_{\xi} x_{\xi}=0, \forall \xi \in F$.

Definição 1.1.6: Um grupo topológico é um grupo $G$ acompanhado de uma topologia em $G$ tal que a operação binária de grupo $G \times G \longmapsto G:(x, y) \longmapsto x y$ e a função inversa $G \longmapsto G: x \longmapsto x^{-1}$ são funções contínuas.

Se $X$ é um conjunto e $G$ é um grupo topológico abeliano, dada uma função $f: X \longmapsto G$, definimos $\operatorname{supp}(f)=\{x \in X: f(x) \neq 0\}$. Denotamos dom $(f)$ pelo domínio da função f. Se $\left\{x_{\xi}: \xi<\kappa\right\}$ é sequência em $G$ e $f \in\left([\kappa]^{<\omega}\right)^{\omega}=\left\{h: \omega \longmapsto[\kappa]^{<\omega}: h\right.$ é função\}, então definimos $x_{f(n)}=\sum_{\xi \in f(n)} x_{\xi}, \forall n \in \omega$. Podemos então considerar a sequência $\left\{x_{f(n)}: n \in \omega\right\} \subseteq G$.

$\mathrm{O}$ ordinal $2=\{0,1\}$ é um grupo topológico abeliano com $0+0=1+1=0$ e $0+1=1+0=1$, acrescido da topologia discreta. Se $X$ é qualquer conjunto, então $2^{X}$ (o conjunto de todas as funções de $X$ em 2) é um grupo topológico compacto quando dada a estrutura de produto de grupos e a topologia de Tychonoff. O elemento indentidade deste grupo será a função nula. Esta será representada por 0, quando não houver perigo 
de confusão. Este tipo de grupo topológico booleano aparecerá com frequência nesta dissertação.

Lema 1.1.3: Em um grupo topológico $G$, se $p$ é ultrafiltro e $\left\{x_{n}: n \in \omega\right\},\left\{y_{n}: n \in \omega\right\}$ são duas sequências em $G$ tais que $x=p-\lim \left\{x_{n}: n \in \omega\right\}$ e $y=p-\lim \left\{y_{n}: n \in \omega\right\}$, então $x+y=p-\lim \left\{x_{n}+y_{n}: n \in \omega\right\}$.

Demonstração: Seja $V$ vizinhança qualquer de $x+y$. Como $+: G \times G \longmapsto G$ é função contínua temos que $+^{-1}(V)$ é um aberto em $G \times G$. Portanto, existem abertos $V_{x}$ e $V_{y}$ de $G$ tais que $x \in V_{x}, y \in V_{y}$ e $V_{x} \times V_{y} \subseteq+^{-1}(V)$ e, portanto, $V_{x}+V_{y} \subseteq V$. Como $x=p-\lim \left\{x_{n}: n \in \omega\right\}$ e $y=p-\lim \left\{y_{n}: n \in \omega\right\}$ temos que $A_{x}=\left\{n \in \omega: x_{n} \in V_{x}\right\}$ e $A_{y}=\left\{n \in \omega: x_{n} \in V_{y}\right\}$ são elementos de $p$. Daí, $\left\{n \in \omega: x_{n}+y_{n} \in V\right\} \supseteq$ $\supseteq\left\{n \in \omega: x_{n}+y_{n} \in V_{x}+V_{y}\right\} \supseteq A_{x} \cap A_{y}$ e, portanto, $x+y=p-\lim \left\{x_{n}+y_{n}: n \in \omega\right\}$.

Lema 1.1.4: Em um grupo topológico $G$, se $p$ é ultrafiltro e $\left\{x_{n}: n \in \omega\right\},\left\{y_{n}: n \in \omega\right\}$ são duas sequências em $G$ tais que $x=p-\lim \left\{x_{n}: n \in \omega\right\}$ e $\left\{n \in \omega: x_{n}=y_{n}\right\}=\Omega \in p$, então $x=p-\lim \left\{y_{n}: n \in \omega\right\}$.

Demonstração: Seja $V$ vizinhança aberta de $x$. Como $x=p-\lim \left\{x_{n}: n \in \omega\right\}$ temos que existe $A \in p$ tal que $A=\left\{n \in \omega: x_{n} \in V\right\}$. Daí, podemos afirmar que $\left\{n \in \omega: y_{n} \in V\right\} \supseteq A \cap \Omega$, elemento de $p$. Logo, $x=p-\lim \left\{y_{n}: n \in \omega\right\}$.

Lema 1.1.5: Seja $\prod_{\alpha \in I} X_{\alpha}$ produto infinito de espaços topológicos e $p$ um ultrafiltro qualquer. Se $\left\{x_{n}: n \in \omega\right\}$ é sequência em $\prod_{\alpha \in I} X_{\alpha}$, então vale $p-\lim \left\{x_{n}: n \in \omega\right\}=x$ se, e somente se, $p-\lim \left\{\pi_{\alpha}\left(x_{n}\right): n \in \omega\right\}=\pi_{\alpha}(x), \forall \alpha \in I$, sendo $\pi_{\alpha}: \prod_{\beta \in I} X_{\beta} \longmapsto X_{\alpha}$ a projeção canônica.

Demonstração: Usando o fato de que cada projeção $\pi_{\alpha}: \prod_{\beta \in I} X_{\beta} \longmapsto X_{\alpha}$ é contínua 
obtemos a implicação direta. Para obter a implicação inversa usamos o fato de que a intersecção finita de elementos do ulltrafiltro ainda é elemento do ultrafiltro. Seja $\left\{x_{n}: n \in \omega\right\}$ sequência em $\prod_{\alpha \in I} X_{\alpha}$ tal que $p-\lim \left\{\pi_{\alpha}\left(x_{n}\right): n \in \omega\right\}=\pi_{\alpha}(x), \forall \alpha \in I$. Se $V$ é aberto básico na topologia de Tychonoff em $\prod_{\beta \in I} X_{\beta}$ tal que $x=\left(x_{\alpha}\right)_{\alpha \in I} \in V$, então existe $F \in[I]^{<\omega}$ tal que $V=\prod_{\beta \in F} V_{\beta} \times \prod_{\beta \in I \backslash F} X_{\beta}$, com $V_{\beta}$ vizinhança aberta de $x_{\beta}, \forall \beta \in F$. Como $p-\lim \left\{\pi_{\alpha}\left(x_{n}\right): n \in \omega\right\}=\pi_{\alpha}(x)=x_{\alpha}, \forall \alpha \in I$, temos que $A_{\beta}=\left\{n \in \omega: \pi_{\beta}\left(x_{n}\right) \in V_{\beta}\right\} \in p$ para todo $\beta$ em $F$. Daí, $\bigcap_{\beta \in F} A_{\beta} \in p$ e $\left\{n \in \omega: x_{n} \in V\right\} \supseteq$ $\supseteq \bigcap_{\beta \in F} A_{\beta}$. Logo, $p-\lim \left\{x_{n}: n \in \omega\right\}=x$.

Provaremos agora um lema que será importante mais adiante na discussão sobre grupos $\omega$-limitados gerado por famílias $\omega$-independentes.

Lema 1.1.6: Em um grupo topológico $G$, se $E$ é subgrupo de $G$, então $\bar{E}$ é subgrupo de $G$.

Demonstração: Dado $x \in \bar{E}$, vamos provar que $x^{-1} \in \bar{E}$. Para isso, considere $V$ vizinhança aberta de $x^{-1}$. Como a função inversa $G \longmapsto G: x \longmapsto x^{-1}$ é função contínua temos que a imagem inversa dessa vizinhança que chamaremos de $V^{-1}$ é vizinhança aberta de $x$. Como $E$ é subgrupo temos que $V^{-1} \cap E \neq \emptyset \Longrightarrow V \cap E \neq \emptyset$ e, portanto, $x^{-1} \in \bar{E}$. Considere agora $x, y \in \bar{E}$ e $V$ vizinhança aberta de $x+y$. Como $+: G \times G \longmapsto G$ é função contínua temos que $+^{-1}(V)$ é um aberto no produto $G \times G$. Podemos tomar $V_{x}$, $V_{y}$ vizinhanças abertas de $x$ e $y$ respectivamente tais que $V_{x} \times V_{y}$ está contido em $+^{-1}(V)$. Temos que existem $a, b \in E$ tais que $a \in V_{x}$ e $b \in V_{y}$ e como $E$ é grupo temos que $a+b \in\left(V_{x}+V_{y}\right) \cap E \subseteq V \cap E . \operatorname{Logo}, x+y \in \bar{E}$.

A partir da definição 1.1.6, se $G$ é grupo topológico e $g$ um elemento fixo de $G$, então a função constante $x \longmapsto g$ e a função indentidade $x \longmapsto x$ são funções contínuas de $G$ em $G$. Dessa forma, elas induzem uma função contínua $x \longmapsto(g, x)$ de $G$ em $G \times G$. Compondo 
esta função com a operação binária de grupo obtemos uma função contínua $l_{g}: x \longmapsto g x$ de $G$ em $G$, chamada multiplicação pela esquerda (ou translação pela esquerda) por $g$. Esta função tem inversa $l_{g^{-1}}$, a qual é contínua. Dessa forma, $l_{g}$ é um homeomorfismo em $G$. Similarmente, as tranlações pela direita $r_{g}: x \longmapsto x g$ são homeomorfismos em $G$. Como consequência, temos que $G$ deve ser um espaço homogêneo, isto é, dados $a, b \in G$ existe um homeomorfismo em $G$ que manda $a$ em $b$. Com isso, temos que $G$ parece ser topologicamente o mesmo em todos os pontos. Como consequência temos o:

Lema 1.1.7: Seja $G$ um grupo topológico e $x \in G$ um ponto isolado. Então, $G$ é discreto.

Vamos agora a um lema que usaremos frequentemente no capítulo 2 para provar que um determinado grupo não é enumeravelmente compacto. Geralmente este grupo é construído para ser enumerável, gerando uma contradição ao supormos enumeravelmente compacto. O lema é posto aqui na sua forma geral, para espaços topológicos, pois não faz uso das propriedades de grupo topológico.

Lema 1.1.8: Seja $X$ um espaço topológico infinito, regular, enumeravelmente compacto. Então $X$ tem cardinalidade $\geq 2^{\omega}$.

Demonstração: Como $X$ é infinito e regular podemos tomar dois pontos $x_{0}$ e $x_{1}$ em $X$ separados por abertos disjuntos $A_{0}$ e $A_{1}$ cujos fechos $F_{0}$ e $F_{1}$ sejam ainda disjuntos. Em cada aberto devemos ter pelo menos dois pontos, pois caso contrário, teríamos um ponto isolado no grupo topológico $X$. Pelo lema anterior, teríamos que o grupo $X$ seria discreto, o que contradiz o fato de $X$ ser enumeravelmente compacto, uma vez que $X$ é infinito. Considere, sem perda de generalidade, o caso $A_{0}$. Teremos, então, dois pontos $x_{00}$ e $x_{01}$ em $A_{0}$, e como $X$ é regular, podemos obter dois abertos disjuntos $A_{00}$ e $A_{01}$ cujos fechos $F_{00}$ e $F_{01}$ sejam ainda disjuntos, com $x_{00} \in A_{00}, x_{01} \in A_{01}$ e $F_{00} \cup F_{01} \subseteq F_{0}$. Novamente, podemos 
em cada aberto obtido tomar dois pontos distintos e continuar o processo por indução até encontrarmos $2^{\omega}$ cadeias de fechados da forma $F_{y_{1}} \supseteq F_{y_{2}} \supseteq F_{y_{3}} \supseteq \ldots \supseteq F_{y_{n}} \supseteq \ldots$, com $y_{n}: n \longmapsto 2, \forall n \geq 1$, e $y_{n}=y_{m} \uparrow n$, se $n \leq m$. Como $X$ é enumeravelmente compacto, teremos que cada cadeia enumerável de fechados tem intersecção não vazia e tomando um ponto em cada intersecção temos que esses pontos deverão ser dois a dois distintos. Logo, existem pelo menos $2^{\omega}$ pontos distintos em $X$ e segue-se o lema.

Definição 1.1.7: Um grupo topológico $G$ é grupo de van Douwen se, e somente se, é abeliano, infinito, enumeravelmente compacto e sem sequências não-triviais convergentes.

Lema 1.1.9: Seja $G$ um grupo de van Douwen regular. Então, toda sequência em $G$ tem pelo menos $\mathfrak{c}$ pontos de acumulação.

Demonstração: Seja $\left\{x_{n}: n \in \omega\right\}$ sequência 1-1 em $G$. Como $G$ é enumeravelmente compacto e não possui sequências não triviais convergentes temos que a sequência $X=\left\{x_{n}: n \in \omega\right\}$ possui dois pontos de acumulação $x_{0}$ e $x_{1}$ em $G$ que podem ser separados por abertos disjuntos $A_{0}$ e $A_{1}$ cujos fechos $F_{0}$ e $F_{1}$ sejam ainda disjuntos. Cada aberto determina uma subsequência de $\left\{x_{n}: n \in \omega\right\}$. Para cada subsequência $X \cap A_{i}$, podemos aplicar mesmo argumento usado anteriormente. Se $s \in \omega^{\omega}$, então definimos $X_{s \uparrow n}=X \cap A_{s \uparrow 0} \cap \ldots \cap A_{s \uparrow n}$. Como $G$ é enumeravelmente compacto temos que $\bigcap_{n \in \omega} \overline{X_{s \uparrow n}} \neq \emptyset$. Tomamos então $x_{s} \in \bigcap_{n \in \omega} \overline{X_{s \uparrow n}}$, ponto de acumulação de $\left\{x_{n}: n \in \omega\right\}$. Basta mostrar agora que se $s \neq r$, então $x_{s} \neq x_{r}$. Se $s \neq r$, então existe $m \in \omega$ tal que $s(m) \neq r(m)$. Pela construção, sabemos que $A_{s \uparrow m+1} \cap A_{r \uparrow m+1}=\emptyset$ e que os fechos de $A_{s \uparrow m+1}$ e $A_{r\lceil m+1}$ são ainda disjuntos. Daí, temos que $x_{s} \neq x_{r}$ pois, caso contrário, teríamos o mesmo ponto sendo ponto de acumulação de $A_{s\lceil m+1}$ e $A_{r\lceil m+1}$. 
DEFINIÇÕES E RESULTADOS PRELIMINARES

\section{$1.2 \quad$ Ultrafiltros seletivos}

Aqui, relembramos algumas propriedades de ultrafiltros seletivos incomparáveis que serão necessários para provar o lema 3.1.1, o qual será usado na construção de um grupo de van Douwen na secção 3.2 .

Definição 1.2.1: Dados $p, q \in \omega^{*}$, dizemos que $p \leq_{R K} q$ se existe uma função $f: \omega \longmapsto \omega$ tal que $\beta f(q)=p$, onde $\beta f$ é a extensão de Stone-Cech de $f$. A pré-ordem $\leq_{R K} \mathrm{em} \omega^{*}$ é chamada ordem de Rudin-Keisler.

Lema 1.2.1: Os ultrafiltros $p$ e $q$ são equivalentes na ordem de Rudin-Keisler se existe uma bijeção $f: \omega \longmapsto \omega$ tal que $\beta f(p)=q$.

Ultrafiltros seletivos são os ultrafiltros minimais na ordem de Rudin-Keisler. Listamos abaixo propriedades equivalentes que usaremos nesta dissertação. As demonstrações dessas equivalências podem ser encontradas em [1].

Lema 1.2.2: As propriedades seguintes são equivalentes para $p \in \omega^{*}$ :

i) $p$ é minimal na ordem de Rudin-Keisler;

ii) $p$ é seletivo, ou seja, para cada $f: \omega \longmapsto \omega$ existe $A \in p$ tal que $f \uparrow A$ é constante ou $1-1$

iii) se $\left\{P_{n}: n \in \omega\right\}$ é partição de $\omega$, então existe $m \in \omega$ tal que $P_{m} \in p$ ou existe $B \in p$ tal que $\left|B \cap P_{n}\right|=1$ para cada $n \in \omega ;$

iv) para cada partição $P_{0} \cup P_{1}=[\omega]^{2}$ existem $D \in p$ e $j \in\{0,1\}$ tais que $[D]^{2} \subseteq P_{j}$.

Lema 1.2.3: Todo ultrafiltro seletivo é um $P$-ponto, isto é, se $\left\{A_{n}: n \in \omega\right\} \subseteq p$, então existe $A \in p$ tal que $A \backslash A_{n}$ é finito, $\forall n \in \omega$. 
Demonstração: Faça $B_{0}=\omega$ e $B_{n}=\bigcap_{i \leq n} A_{i}, \forall n \in \omega$. Temos então que $B_{0} \supseteq B_{1} \supseteq$ $\ldots \supseteq B_{n} \supseteq \ldots$ e que $B_{n} \in p, \forall n \in \omega$. Isto induz uma partição em $\omega: C_{n}=B_{n} \backslash B_{n+1}, \forall n \in \omega$. Como $B_{n+1}$ pertence ao ultrafiltro, temos que $C_{n}$ não pertence ao ultrafiltro, $\forall n \in \omega$. Logo, pelo item iii) do lema anterior, existe $A \in p$ tal que $\left|A \cap C_{n}\right|=1$ para cada $n \in \omega$. Daí, $A \backslash A_{n} \subseteq \bigcup_{m<n} A \cap C_{m}$ é finito, $\forall n \in \omega$.

A existência de ultrafiltros seletivos é independente dos axiomas usuais da teoria dos conjuntos: existem $2^{\mathfrak{c}}$ ultrafiltros seletivos com o uso da hipótese do contínuo ou do axioma de Martin e Shelah demonstrou com o uso de forcing que existem modelos sem P-pontos em particular, sem ultrafiltros seletivos.

\section{$1.3 \quad$ Ultraprodutos}

Ultraprodutos foram definidos por Lŏs em 1955. Nesta dissertação, obteremos um grupo de van Douwen $G$ usando um ultrafiltro seletivo e relacionando as sequências em $G$ aos elementos do ultraproduto $\left([\mathfrak{c}]^{<\omega}\right)^{\omega} / p$. O ultraproduto será usado como espaço vetorial sobre o corpo $\{0,1\}$ e com a operação $\triangle$, diferença simétrica de conjuntos. Por $\vec{F}$, denotaremos a função em $\left([\mathfrak{c}]^{<\omega}\right)^{\omega}$ que é constante igual a $F \in[\mathfrak{c}]^{<\omega}$. Se $\alpha$ é ordinal, usaremos $\vec{\alpha}$ no lugar de $\{\vec{\alpha}\}$. Definiremos agora o ultraproduto que necessitamos para essa dissertação.

Primeiramente, observe que dadas duas funções $f, g \in\left([\mathfrak{c}]^{<\omega}\right)^{\omega}$ e $p \in \omega^{*}, f \sim g \Longleftrightarrow$ $\{n \in \omega: f(n)=g(n)\} \in p$ define uma relação de equivalência. Temos então:

Definição 1.3.1: Seja $p \in \omega^{*}$ :

a) Dadas duas funções $f, g \in\left([\mathfrak{c}]^{<\omega}\right)^{\omega}$, dizemos que $\mathrm{f}$ e g são p-equivalentes se, e somente se, o conjunto $\{n \in \omega: f(n)=g(n)\} \in p$; 
b) A classe p-equivalente de $f,[f]_{p}$, é o conjunto de todos os elementos p-equivalentes de $f$;

c) Denote por $\left([\mathfrak{c}]^{<\omega}\right)^{\omega} / p$ o conjunto de todas as classes p-equivalentes. Se $f, g \in$ $\left([\mathfrak{c}]^{<\omega}\right)^{\omega}$, defina $[f]_{p} \triangle[g]_{p}$ como a classe $[f \triangle g]_{p}$, em que $(f \triangle g)(n)=f(n) \triangle g(n), \forall n \in \omega$.

Lema 1.3.1: Seja $p$ um elemento de $\omega^{*}$ :

a) $\mathrm{O}$ conjunto $\left([\mathfrak{c}]^{<\omega}\right)^{\omega} / p$ com a operação $\triangle$ é um espaço vetorial sobre 2 ;

b) Uma família $\left\{\left[g_{\alpha}\right]_{p}: \alpha \in I\right\}$ de classes p-equivalentes de $\left([\mathfrak{c}]^{<\omega}\right)^{\omega} / p$

i) é linearmente independente se, e somente se, para todo $F \in[I]^{<\omega}$ existe $A \in p$ tal que $\triangle\left(\left\{g_{\alpha}(n): \alpha \in F\right\}\right) \neq \emptyset, \forall n \in A$;

ii) gera o espaço vetorial $\left([\mathfrak{c}]^{<\omega}\right)^{\omega} / p$ se, e somente, se para todo $g \in\left([\mathfrak{c}]^{<\omega}\right)^{\omega}$ existe $A \in p$ e $F \in[I]^{<\omega}$ tais que $g(n)=\triangle\left(\left\{g_{\alpha}(n): \alpha \in F\right\}\right), \forall n \in A$.

Demonstração: a) Defina o elemento neutro da adição como a função $\overrightarrow{0}: \omega \longmapsto[c]]^{<\omega}$ tal que $\overrightarrow{0}(n)=\emptyset, \forall n \in \omega$, e a multiplicação por escalar $0 \cdot[f]_{p}=\overrightarrow{0}$ e $1 \cdot[f]_{p}=[f]_{p}$. O elemento inverso da adição de $[f]_{p}$ será $[f]_{p}$.

b) - i) Se $\left\{\left[g_{\alpha}\right]_{p}: \alpha \in I\right\}$ é l.i., então dado $F \in[I]^{<\omega}$ devemos ter $\triangle_{\alpha \in F}\left(\left[g_{\alpha}\right]_{p}\right) \neq[\overrightarrow{0}]_{p}$, ou seja, $\left\{n \in \omega: \triangle_{\alpha \in F}\left(g_{\alpha}(n)\right)=\emptyset\right\} \notin p$. Como $p$ é ultrafiltro, temos que $A=\left\{n \in \omega: \triangle_{\alpha \in F}\left(g_{\alpha}(n)\right) \neq \emptyset\right\} \in p$.

Por outro lado, suponha, por contradição que existe $F \in[I]^{<\omega}$ tal que $\triangle_{\alpha \in F}\left(\left[g_{\alpha}\right]_{p}\right)=$ $[\overrightarrow{0}]_{p}$. Logo, existe $B \in p$ tal que $B=\left\{n \in \omega: \triangle_{\alpha \in F}\left(g_{\alpha}(n)\right)=\emptyset\right\}$.

Mas por hipótese, temos que $A=\left\{n \in \omega: \triangle_{\alpha \in F}\left(g_{\alpha}(n)\right) \neq \emptyset\right\} \in p$. Assim, $A \cap B=\emptyset \in p$, o que é absurdo.

ii) Basta notar que $\left\{n \in \omega: g(n)=\triangle\left(\left\{g_{\alpha}(n): \alpha \in F\right\}\right)\right\} \in p$ se, e somente se, $[g]_{p}=\triangle\left(\left\{\left[g_{\alpha}\right]_{p}: \alpha \in F\right\}\right)$.

Agora consideremos um resultado que será importante na construção do grupo de van 
Douwen usado nesta dissertação.

Lema 1.3.2: Seja $p \in \omega^{*}$ ultrafiltro seletivo. Então,

$$
\left([\mathfrak{c}]^{<\omega}\right)^{\omega} / p=\left\{[f]_{p}: f \in\left([\mathfrak{c}]^{<\omega}\right)^{\omega} \text { é injetora }\right\} \cup\left\{[\vec{F}]_{p}: F \in[\mathfrak{c}]^{<\omega}\right\} .
$$

Demonstração: Se $g \in\left([\mathfrak{c}]^{<\omega}\right)^{\omega}$, então como a imagem de $g$ é enumerável, com o uso de uma bijeção entre a imagem de $g$ e $\omega$ podemos pelo item ii) do Lema 1.2.2 encontrar $A \in p$ tal que $g\lceil A$ é constante ou injetora.

\subsection{Famílias independentes e subgrupos $\omega$-limitados.}

No capítulo 2, precisaremos de uma família $\omega$-independente de tamanho $\mathfrak{c}$ em $\mathfrak{c}$.

Definição 1.4.1: $\mathcal{A}$ é uma família $\omega$-independente de tamanho $\mathfrak{c}$ em $\mathfrak{c}$ se $\mathcal{A}$ é uma família de subconjuntos de $\mathfrak{c}$ satisfazendo: se $\mathcal{A}^{\prime}, \mathcal{A}^{\prime \prime} \in[\mathcal{A}]^{\leq \omega}$ são disjuntos, então $\left|\cap \mathcal{A}^{\prime} \backslash \bigcup \mathcal{A}^{\prime \prime}\right|=\mathfrak{c}$.

Tais famílias existem e um fácil exemplo pode ser obtido como se segue:

Exemplo 1.4.1: Para $\alpha \in \mathfrak{c}$, considere o conjunto $A_{\alpha}=\left\{F \in[\mathfrak{c} \times \mathfrak{c}]^{\leq \omega}: F \cap(\{\alpha\} \times \mathfrak{c}) \neq\right.$ $\emptyset\}$. Então, fazemos $\mathcal{A}=\left\{A_{\alpha}: \alpha \in \mathfrak{c}\right\} \subseteq[\mathfrak{c} \times \mathfrak{c}]^{\leq \omega}$, conjunto de cardinalidade $\mathfrak{c}$. Temos que $\mathcal{A}$ satisfaz

$$
\text { se } K \in[\mathfrak{c}]^{\leq \omega} \text {, então }\left|\bigcap_{\alpha \in K} A_{\alpha} \backslash \bigcup_{\alpha \in \mathfrak{c} \backslash K} A_{\alpha}\right|=\mathfrak{c} \text {. }
$$

Vê-se facilmente que $\mathcal{A}$ satisfaz a definição, já que dados $\mathcal{A}^{\prime}, \mathcal{A}^{\prime \prime} \in[\mathcal{A}] \leq \omega$ disjuntos, podemos fazer $K=\left\{\alpha \in \mathfrak{c}: A_{\alpha} \in \mathcal{A}^{\prime}\right\}$ que teremos $\left\{\alpha \in \mathfrak{c}: A_{\alpha} \in \mathcal{A}^{\prime \prime}\right\} \subseteq \mathfrak{c} \backslash K$. Também é claro que $\mathcal{A}$ não foi construído em $\mathfrak{c}$, mas em $[\mathfrak{c} \times \mathfrak{c}]^{\leq \omega}$, conjunto de cardinalidade $\mathfrak{c}$. 
Considere agora $\mathcal{B}$, família $\omega$-independente de tamanho $\mathfrak{c}$ em um conjunto $X$ qualquer. Podemos indentificar $\mathcal{B}$ com o seu conjunto de funções características $\mathcal{X}_{B}: X \longmapsto 2$, com $B \in \mathcal{B}$. Assim, podemos pensar em $\mathcal{B}$ como contido no grupo $2^{X}$. Seja $G=\langle\mathcal{B}\rangle$, o grupo gerado por $\mathcal{B}$. Considere

$$
\begin{aligned}
G^{+} & =\bigcup\left\{\bar{E}: E \in[G]^{\leq \omega}\right\} \\
& =\bigcup\left\{\bar{E}: E \in[G]^{\leq \omega} \text { e } E \text { é subgrupo de } G\right\} .
\end{aligned}
$$

Vale a igualdade acima, pois se $E \in[G]^{\leq \omega}$, então $\langle E\rangle$, subgrupo gerado por $E$, ainda será enumerável e é claro que $\bar{E} \subseteq \overline{\langle E\rangle}$. Dessa forma, $G^{+}$é subgrupo de $2^{X}$ e temos que se $E \subseteq G^{+}$é enumerável, então $\bar{E} \subseteq G^{+}$. Segue-se que $G^{+}$é $\omega$-limitado, ou seja, $G^{+}$é espaço topológico tal que se $E \subseteq G^{+}$é enumerável, então $\bar{E}$ é compacto em $G^{+}$. É fácil provar que $G^{+}$é o menor subgrupo $\omega$-limitado de $2^{X}$ que contém $\mathcal{B}$. Isto leva à definição abaixo.

Definição 1.4.2: Seja $\mathcal{B}$ família $\omega$-independente de tamanho $\mathfrak{c}$ em um conjunto $X$ qualquer e $G=\langle\mathcal{B}\rangle$, o grupo gerado por $\mathcal{B}$ em $2^{X}$. Definimos $G^{+}=\bigcup\left\{\bar{E}: E \in[G]^{\leq \omega}\right\}$ como o subgrupo $\omega$-limitado gerado por $\mathcal{B}$.

Nas secções 2 e 3 do capítulo 2, precisaremos de dois resultados sobre famílias $\omega$ independentes que colocaremos sob a forma de dois lemas.

Lema 1.4.1: Seja $G^{+}$é o subgrupo $\omega$-limitado gerado pela família $\omega$-independente $\mathcal{B}$ e $x \in G^{+} \backslash\{\underline{0}\}$. Então, podemos obter $\mathcal{B}_{0}, \mathcal{B}_{1}, \mathcal{B}_{2} \in[\mathcal{B}] \leq \omega$ tais que $\bigcap \mathcal{B}_{0} \backslash \bigcup \mathcal{B}_{1} \subseteq \operatorname{supp}(x) \subseteq$ $\subseteq \cup \mathcal{B}_{2}$ e $\mathcal{B}_{0} \cap \mathcal{B}_{1}=\emptyset$.

Demonstração: Seja $E \subseteq G=\langle\mathcal{B}\rangle$ enumerável tal que $x \in \bar{E}$. Para cada $e \in E$, existe um subconjunto finito $F_{e} \subseteq \mathcal{B}$ tal que $e=\sum_{B \in F_{e}} B$. Faça $\mathcal{B}_{2}=\left\{F_{e}: e \in E\right\}$. Agora, se 
$\alpha \notin \bigcup \mathcal{B}_{2}$, então $e(\alpha)=0, \forall e \in E$. Como $x \in \bar{E}$, temos que $x(\alpha)=0$. Portanto, $\operatorname{supp}(x) \subseteq$ $\subseteq \cup \mathcal{B}_{2}$. Por outro lado, escolha $\alpha \in \operatorname{supp}(x)$. O conjunto $U=\left\{y \in 2^{X}: y(\alpha)=1\right\}$ é uma vizinhança de $x$ em $2^{X}$ e, portanto, $x \in \overline{U \cap E}$. Para $e \in U \cap E$, faça $F_{e}^{+}=\left\{B \in F_{e}: \alpha \in B\right\}$ e $F_{e}^{-}=F_{e} \backslash F_{e}^{+}$. Temos então que se $\mathcal{B}_{0}=\bigcup\left\{F_{e}^{+}: e \in U \cap E\right\}$ e $\mathcal{B}_{1}=\bigcup\left\{F_{e}^{-}: e \in U \cap E\right\}$, então $\alpha \in \bigcap \mathcal{B}_{0} \backslash \bigcup \mathcal{B}_{1}$. Agora, seja $\gamma \in \bigcap \mathcal{B}_{0} \backslash \cup \mathcal{B}_{1}$ e $e \in U \cap E$. Então, $B \in F_{e}^{+}$implica em $\gamma \in B$ e $B \in F_{e}^{-}$implica em $\gamma \notin B$. Segue-se que $e(\gamma)=e(\alpha)=1$. Como $x \in \overline{U \cap E}$, temos também que $x(\gamma)=1$. Logo, $\cap \mathcal{B}_{0} \backslash \cup \mathcal{B}_{1} \subseteq \operatorname{supp}(x)$.

Lema 1.4.2: Seja $\mathcal{B}$ uma família $\omega$-independente em $\mathfrak{c}$. Para cada $B \in \mathcal{B}$, seja $X_{B}$ subconjunto de $\mathfrak{c}$ tal que $\left|X_{B} \triangle B\right|<\mathfrak{c}$. Então, $\left\{X_{B}: B \in \mathcal{B}\right\}$ é $\omega$-independente.

Demonstração: Se $\mathcal{B}^{\prime}, \mathcal{B}^{\prime \prime} \subseteq \mathcal{B}$ são famílias enumeráveis e disjuntas, faça $I=$ $\bigcup_{B \in \mathcal{B}^{\prime} \cup \mathcal{B}^{\prime \prime}} X_{B} \triangle B$. Então, como a cofinalidade de $\mathfrak{c}$ é maior do que $\omega$ e $\left|\mathcal{B}^{\prime} \cup \mathcal{B}^{\prime \prime}\right|=\omega$ temos que $|I|<\mathfrak{c} . \operatorname{Logo},\left(\bigcap_{B \in \mathcal{B}^{\prime}} X_{B} \backslash \bigcup_{B \in \mathcal{B}^{\prime \prime}} X_{B}\right) \backslash I=\left(\bigcap \mathcal{B}^{\prime} \backslash \bigcup \mathcal{B}^{\prime \prime}\right) \backslash I$ tem cardinalidade $\mathfrak{c}$.

\subsection{Axioma de Martin para ordens parciais enumeráveis $\left(\mathrm{MA}_{\mathrm{e}}\right)$}

Nesta dissertação, usaremos uma forma enumerável do Axioma de Martin - que denotaremos por $\mathbf{M} \mathbf{A}_{\mathrm{e}}$, na construção de um subgrupo de $2^{\mathfrak{c}}$ que provará ser útil na obtenção de dois subgrupos enumeravelmente compactos cujo produto não é enumeravelmente compacto. Ainda, poderemos com a ajuda do $\mathbf{M} \mathbf{A}_{\mathrm{e}}$ construir um subgrupo de $2^{\mathfrak{c}} \times 2^{\mathfrak{c}}$ que será útil na construção de um grupo topológico enumeravelmente compacto $H$ cujo quadrado não é enumeravelmente compacto. Estes exemplos podem ser encontrados no capítulo 2. O leitor que se interessar pela versão geral do Axioma de Martin (MA) pode consultar [7]. Vamos ao enunciado de $\mathbf{M} \mathbf{A}_{\mathrm{e}}$ : 
Definição 1.5.1 $\left(\mathbf{M A}_{\mathrm{e}}\right)$ : Se $P$ é uma ordem parcial enumerável e se $\mathcal{D}$ é uma coleção de cardinalidade menor que $\mathfrak{c}$ de conjuntos densos em $P$, então existe um filtro $G$ em $P$ que intersecta todo elemento de $\mathcal{D}$.

Aqui, um conjunto $D \subseteq P$ é denso se, e somente se, para todo $p \in P$, existe $q \in D$ tal que $q \leq p$. Um conjunto $G \subseteq P$ é filtro se, e somente se, para todo $p \in G$ e $q \in P$, $p \leq q \Longrightarrow q \in G$ e se para todo $p, q \in G$, existe $r \in G$ tal que $r \leq p$ e $r \leq q$.

Nesta dissertação, devemos usar $\mathbf{M} \mathbf{A}_{\mathrm{e}}$ com ordens parciais que são subconjuntos de $F n(I, x)$, com $I$ enumerável e $x$ finito. Aqui,

$$
F n(I, x)=\left\{p: \operatorname{dom}(p) \in[I]^{<\omega} \text { e } \operatorname{Im}(p) \subseteq x\right\}
$$

ordenada por inclusão inversa: $p \leq q \Longleftrightarrow p \supseteq q$. Neste caso, um conjunto $D$ é denso se cada $p$ tem uma extensão $q \in D$, e um conjunto $G$ é um filtro se é fechado com relação a tomar restrições e se para dois elementos quaisquer $p, q \in G$, existe uma função $r \in G$ que estende $p$ e $q$. Se $G$ é filtro, então $\bigcup G$ é uma função. Temos que $D_{i}=\{p: i \in d o m(p)\}$ é denso, $\forall i \in I$. Neste caso, se $G \cap D_{i} \neq \emptyset$ para todo $i \in I$, então $\operatorname{dom}(\bigcup G)=I$. 


\section{Capítulo 2}

\section{Primeiros contra-exemplos para a produtividade da compacidade enumerável.}

Neste capítulo podem ser encontrados os primeiros contra-exemplos mais importantes para a produtividade da compacidade enumerável em grupos topológicos. Todos os exemplos neste capítulo depedem de alguma versão do Axioma de Martin (MA).

\subsection{Exemplo de van Douwen}

Na secção 3.1 do próximo capítulo construiremos um grupo de van Douwen booleano. Vamos utilizar este grupo nesta secção antes de construí-lo por uma questão histórica: o exemplo de dois grupos enumeravelmente compactos $H_{0}$ e $H_{1}$ cujo produto $H_{0} \times H_{1}$ não é enumeravelmente compacto obtido por van Douwen em [2] usava como hipótese a existência 
do grupo de van Douwen, que foi obtido em [2] com a ajuda do Axioma de Martin. A construção do grupo de van Douwen booleano que aparece na secção 3.1 do próximo capítulo foi obtida posteriormente em [5] usando como hipótese apenas a existência de um ultrafiltro seletivo. Optamos por não apresentar a construção do grupo de van Douwen usando o Axioma de Martin, mas por outro lado apresentamos a construção do exemplo para efeito comparativo com as técnicas mais elaboradas que se seguiram.

Lema 2.1.1: Seja $H$ um grupo de van Douwen booleano. Então, existem subgrupos enumeravelmente compactos $H_{0}$ e $H_{1}$ de $H$ tais que $\left|H_{0} \cap H_{1}\right|=\omega$.

Demonstração: Defina uma coleção de ordinais $\left\{\sigma_{\alpha}: \alpha \leq \mathfrak{c}\right\}$ tais que $\sigma_{0}=\omega$, $\sigma_{\alpha+1}=\sigma_{\alpha}+\left|\sigma_{\alpha}\right|, \forall \alpha<\mathfrak{c}$, e $\sigma_{\lambda}=\sup _{\alpha<\lambda} \sigma_{\alpha}$, quando $\lambda \leq \mathfrak{c}$ é ordinal limite. Note que $\sigma_{\alpha}<\mathfrak{c}, \forall \alpha<\mathfrak{c}$, uma vez que $\sigma_{\alpha}<\kappa$ para $\alpha<\kappa$, se $\kappa \leq \mathfrak{c}$ é regular. Note também que $\sigma_{\mathfrak{c}}=\mathfrak{c}$. Enumere $[\mathfrak{c}]^{\omega}$ como $\left\{I_{\alpha}: \alpha<\mathfrak{c}\right\}$ de tal forma que $I_{\alpha} \subseteq \sigma_{\alpha}, \forall \alpha<\mathfrak{c}$.

Construiremos duas sequências estritamente crescentes $\left\{H_{i, \alpha}: \alpha \leq \mathfrak{c}\right\}, \forall i \in 2$, de subgrupos de $H$. Nossos $H_{0}$ e $H_{1}$ serão ao final $H_{0, \mathfrak{c}}$ e $H_{1, \mathfrak{c}}$. Cada $H_{i, \alpha}$ será indexado por $H_{i, \alpha}=\left\{y_{i, \xi}: \xi \in \sigma_{\alpha}\right\}$. Estas sequências deverão satisfazer as seguintes condições:

(1) se $\xi<\eta<\sigma_{\alpha}$, então $y_{i, \xi} \neq y_{i, \eta}, \forall i \in 2, \forall \alpha \leq \mathfrak{c}$;

(2) $H_{0, \alpha} \cap H_{1, \alpha}=H_{0,0}, \forall \alpha \leq \mathfrak{c}$ (Isto assegura que $\left|H_{0} \cap H_{1}\right|=\omega$ );

(3) $\left\{y_{i, \xi}: \xi \in I_{\alpha}\right\}$ tem ponto de acumulação em $H_{i, \alpha+1}, \forall \alpha<\mathfrak{c}$ (Isto assegura que $H_{0}$ e $H_{1}$ serão enumeravelmente compactos).

A construção e o indexamento de $H_{0, \lambda}$ e $H_{1, \lambda}$ para o caso $\lambda$ ordinal limite $\leq \mathfrak{c}$, incluindo $\lambda=0$, é direta. Pois, se $\alpha<\lambda$, então $\sigma_{\alpha}$ é conhecido e daí temos definidos $\sigma_{\lambda}=\sup _{\alpha<\lambda} \sigma_{\alpha}$ e $H_{i, \lambda}=\left\{y_{i, \xi}: \xi \in \sigma_{\lambda}\right\}$.

Agora, seja $\alpha<\mathfrak{c}$ e assuma conhecido $H_{i, \alpha}$. Para todo $i<2$, tome um ponto de acumulação $c_{i}$ de $\left\{y_{i, \xi}: \xi \in I_{\alpha}\right\}$ tal que $c_{0} \notin H_{0, \alpha}+H_{1, \alpha}$ e $c_{1} \notin\left\{0, c_{0}\right\}+H_{0, \alpha}+H_{1, \alpha}$, onde $\left\{0, c_{0}\right\}$ é subgrupo do grupo booleano $H$. Isto é possível, uma vez que $\left|\sigma_{\alpha}\right|<\mathfrak{c}$ e 
$\left\{y_{i, \xi}: \xi \in I_{\alpha}\right\}$ é infinito pelo item (1) e, portanto, tem pelo menos c pontos de acumulação, de acordo com o lema 1.1.8.

Defina $H_{i, \alpha+1}=\left\{0, c_{i}\right\}+H_{i, \alpha}, \forall i<2$.

Uma vez que $\left|H_{i, \alpha}\right|=\left|\sigma_{\alpha}\right|$ e $c_{i} \notin H_{i, \alpha}$, teremos $\left|H_{i, \alpha+1}-H_{i, \alpha}\right|=\left|c_{i}+H_{i, \alpha}\right|=\left|\sigma_{\alpha}\right|$, $\forall i<2$. Portanto, $H_{i, \alpha+1}-H_{i, \alpha}$ pode ser indexado, sem repetições, por $\left\{y_{i, \alpha}: \sigma_{\alpha} \leq \xi \leq\right.$ $\left.\sigma_{\alpha+1}\right\}$. Isto cuida do item (1).

Para o item (2), observe que $\left(c_{0}+H_{0, \alpha}\right) \cap H_{1, \alpha}=\emptyset$, já que $c_{0} \notin H_{0, \alpha}+H_{1, \alpha}$ e $H_{0, \alpha+1} \cap\left(c_{1}+H_{1, \alpha}\right)=\emptyset$, pois $c_{1} \notin H_{0, \alpha+1}+H_{1, \alpha}$. Portanto, $H_{0, \alpha+1} \cap H_{1, \alpha+1}=H_{0, \alpha+1} \cap H_{1, \alpha}=$ $H_{0, \alpha} \cap H_{1, \alpha}=H_{0,0 .}$

Finalmente, está claro que vale (3). Isto completa a construção.

Exemplo 2.1.1: Seja $H$ um grupo de van Douwen booleano. Então, existem subgrupos enumeravelmente compactos $H_{0}$ e $H_{1}$ de $H$ tais que $H_{0} \times H_{1}$ não é enumeravelmente compacto.

Demonstração: Suponha, por contradição, que $H_{0} \times H_{1}$ é enumeravelmente compacto. Sabemos que $H_{0} \times H_{1}$ possui um subgrupo fechado infinito enumerável, chamado "diagonal": $\triangle=\left\{(x, y) \in H_{0} \times H_{1}: x=y\right\}=\left\{(x, x): x \in H_{0} \cap H_{1}\right\}$. Este é fechado, pois $H$ é Hausdorff. Teremos então que $\triangle$ será enumeravelmente compacto. Mas de acordo com o lema 1.1.8 não existe grupo topológico infinito enumerável que seja enumeravelmente compacto. Logo, $H_{0} \times H_{1}$ não pode ser enumeravelmente compacto.

\subsection{Primeiro exemplo de Hart e van Mill}

Este exemplo encontra-se originalmente em [6]. Usando a versão enumerável do Axioma de Martin $\left(\mathbf{M A}_{\mathrm{e}}\right)$ descrita no capítulo 1 são construídos dois subgrupos enumeravelmente 
compactos $H_{0}$ e $H_{1}$ de $2^{\mathfrak{c}}$ tais que $H_{0} \times H_{1}$ não é enumeravelmente compacto. Para esta secção, denotaremos por $\underline{0}$ a função nula $\underline{0}: \mathfrak{c} \longmapsto 2$ que leva todo ordinal $\xi \in \mathfrak{c}$ no zero. $\mathrm{O}$ próximo lema é responsável por construir 3 subgrupos $D, G_{0}$ e $G_{1}$ de $2^{\mathfrak{c}}$ tais que $H_{0}=D+G_{0}$ e $H_{1}=D+G_{1}$.

Lema 2.2.1: $\left(\mathbf{M A}_{\mathrm{e}}\right)$ Existem 3 subgrupos $D, G_{0}$ e $G_{1}$ de $2^{\mathfrak{c}}$ que satisfazem:

(i) $D$ é enumerável;

(ii) $G_{0}$ e $G_{1}$ são $\omega$-limitados;

(iii) $G_{0} \cap G_{1}=\{\underline{0}\}=D \cap\left(G_{0}+G_{1}\right)$;

(iv) se $E \subseteq D$ é infinito então $E$ possui pontos de acumulação em $G_{0}$ e $G_{1}$.

(Na verdade, $\mathbf{M A}_{\mathrm{e}}$ é usado somente na construção de $D$ ).

Demonstração: Do exemplo 1.4 .1 das preliminares tomamos nossa família $\mathcal{A}$ $\omega$-independente em $\mathfrak{c}$ e a particionamos em duas famílias disjuntas $\mathcal{A}_{0}$ e $\mathcal{A}_{1}$ de tamanho $\mathfrak{c}$. Enumeramos essas famílias como $\left\{A_{\alpha}^{0}: \omega \leq \alpha<\mathfrak{c}\right\}$ e $\left\{A_{\alpha}^{1}: \omega \leq \alpha<\mathfrak{c}\right\}$ respectivamente, sem repetições.

Enquanto construímos $D$ devemos modificar um pouco os conjuntos $A_{\alpha}^{0}$ e $A_{\alpha}^{1}$ a fim de obter conjuntos $B_{\alpha}^{0}$ e $B_{\alpha}^{1}$ tais que $\left|B_{\alpha}^{i} \triangle A_{\alpha}^{i}\right|<\mathfrak{c}$ para todo $\alpha$ e $i$. Faremos $\mathcal{B}_{i}=\left\{B_{\alpha}^{i}: \omega \leq \alpha<\mathfrak{c}\right\} \quad(i \in 2)$ e $\mathcal{B}=\mathcal{B}_{0} \cup \mathcal{B}_{1}$. Pelo lema 1.4.1, $\mathcal{B}$ ainda será $\omega$ independente. Finalmente, faremos $G_{0}$ e $G_{1}$ subgrupos $\omega$-limitados de $2^{\mathfrak{c}}$ gerados por $\mathcal{B}_{0}$ e $\mathcal{B}_{1}$, respectivamente.

Vejamos como cuidamos do item (iii). Tome $x \in G_{0} \backslash\{\underline{0}\}$ e $y \in G_{1} \backslash\{\underline{0}\}$. Pelo lema 1.4.2, existem K, L e M subconjuntos enumeráveis de $[\omega, \mathfrak{c})$ tais que $\bigcap_{\alpha \in K} B_{\alpha}^{0} \backslash \bigcup_{\alpha \in L} B_{\alpha}^{0} \subseteq \operatorname{supp}(x)$ e $\operatorname{supp}(y) \subseteq \bigcup_{\alpha \in M} B_{\alpha}^{1}$. Mas então, $\bigcap_{\alpha \in K} B_{\alpha}^{0} \backslash\left(\bigcup_{\alpha \in L} B_{\alpha}^{0} \cup \bigcup_{\alpha \in M} B_{\alpha}^{1}\right)$ tem cardinalidade $\mathfrak{c}$ - já que $\mathcal{B}$ é $\omega$-independente e $L \cup M$ é enumerável, e está contido em $\operatorname{supp}(x) \backslash \operatorname{supp}(y)$. Segue-se que $G_{0} \cap G_{1}=\{\underline{0}\}$.

Agora, veremos do que necessitamos para fazer $D \cap\left(G_{0}+G_{1}\right)=\{\underline{0}\}$. Primeiramente, 
observe que $G_{0}+G_{1}$ é o subgrupo $\omega$-limitado de $2^{\mathfrak{c}}$ gerado por $\mathcal{B}$. De fato, se $G$ é o menor subgrupo $\omega$-limitado que contém $\mathcal{B}$ então $G$ deve conter $G_{0}+G_{1}$ : se $g_{0}+g_{1} \in G_{0}+G_{1}$, considere $E_{0}$ e $E_{1}$ pertencentes a $\left[\left\langle\mathcal{B}_{0}\right\rangle\right]^{\leq \omega}$ e $\left.\left[\left\langle\mathcal{B}_{1}\right\rangle\right]\right]^{\leq \omega}$ respectivamente, com $g_{0} \in \overline{E_{0}}$ e $g_{1} \in \overline{E_{1}}$. Como $\mathcal{B}_{0}, \mathcal{B}_{1} \subseteq \mathcal{B}$, temos que $E_{0}$ e $E_{1}$ pertecem a $[\langle\mathcal{B}\rangle] \leq \omega$ de forma que $g_{0}$ e $g_{1}$ pertecem ao subgrupo $G$, donde $g_{0}+g_{1} \in G$. Logo, como $G_{0}+G_{1}$ é subgrupo $\omega$-limitado temos que $G=G_{0}+G_{1}$. Agora, se $x \in\left(G_{0}+G_{1}\right) \backslash\{\underline{0}\}$ então pelo lema 1.4.2, existe uma subfamília enumerável $\mathcal{B}^{\prime} \subseteq \mathcal{B} \operatorname{com} \operatorname{supp}(x) \subseteq \mathcal{B}^{\prime}$. Assim, uma condição suficiente para $D \cap\left(G_{0}+G_{1}\right)=\{\underline{0}\}$ seria que para todo $d \in D \backslash\{\underline{0}\}$ e para todo $\mathcal{B}^{\prime} \in[\mathcal{B}]^{\leq \omega}$ tenhamos $\operatorname{supp}(d) \backslash \bigcup \mathcal{B}^{\prime} \neq \emptyset$. Entretanto, nós saberemos somente quem é $\mathcal{B}$ no final da construção do subgrupo $D$, de forma que precisaremos de uma outra condição suficiente envolvendo $\mathcal{A}$. Considere a condição:

(v) para todo $d \in D \backslash\{\underline{0}\}$ e para todo $\mathcal{A}^{\prime} \in[\mathcal{A}]^{\leq \omega}$ temos $\left|\operatorname{supp}(d) \backslash \bigcup \mathcal{A}^{\prime}\right|=\mathfrak{c}$.

Pois, se $\mathcal{A}^{\prime}$ é subfamília enumerável de $\mathcal{A}$ e $\mathcal{B}^{\prime}$ a subfamília de $\mathcal{B}$ correspondente, faça $I=\bigcup_{A \in \mathcal{A}^{\prime}} A \triangle B_{A}$, onde $B_{A}$ é tal que $B_{A} \in \mathcal{B}^{\prime}$ e $\left|A \triangle B_{A}\right|<\mathfrak{c}$. Então, como a cofinalidade de $\mathfrak{c}$ é maior do que $\omega$ e $\left|\mathcal{A}^{\prime}\right|=\omega$ temos que $|I|<\mathfrak{c}$. Logo, $\left|\operatorname{supp}(d) \backslash \bigcup \mathcal{A}^{\prime}\right|=\mathfrak{c}$ implica em $\left|\left(\operatorname{supp}(d) \backslash \bigcup \mathcal{A}^{\prime}\right) \backslash I\right|=\mathfrak{c}$ e, portanto, $\operatorname{supp}(d) \backslash \bigcup \mathcal{B}^{\prime} \neq \emptyset$.

Finalmente, descreveremos como será o subconjunto $D$. Primeiramente, definimos o subconjunto $I=\left\{x \in 2^{\omega}: \operatorname{supp}(x)\right.$ é finito $\} \subseteq 2^{\omega}$ que indexará o subgrupo $D$. Assim, faremos na construção abaixo $D=\left\{d_{x}: x \in I\right\}$ de tal forma que para todo $x \in I, d_{x} \uparrow \omega=x$ e para todo $x, y \in I, d_{x}+d_{y}=d_{x+y}$, o que garante que $D$ será subgrupo. Dessa forma, já temos os itens (i) e (ii) e já indicamos como chegaremos ao item (iii) através de (v).

Para o item (iv), enumeraremos $[I]^{\omega} \operatorname{como}\left\{E_{\alpha}: \omega \leq \alpha<\mathfrak{c}\right\}$. Durante a nossa construção do subgrupo $D$ tomaremos no passo $\alpha$ um ponto de acumulação $y_{\alpha}$ de $\left\{d_{x} \uparrow \alpha: x \in E_{\alpha}\right\}$ e faremos $B_{\alpha}^{i}=y_{\alpha} \cup A_{\alpha}^{i} \uparrow[\alpha, \mathfrak{c}), \forall i \in 2$. Durante o resto da construção de $D$ asseguraremos que $B_{\alpha}^{0}$ e $B_{\alpha}^{1}$ serão pontos de acumulação de $\left\{d_{x}: x \in E_{\alpha}\right\}$. Além disso, temos que cuidar do 
item $(\mathrm{v})$. Neste caso, considere $\left\{\left(x_{\alpha}, \mathfrak{C}_{\alpha}\right): \omega \leq \alpha<\mathfrak{c}\right\}$, enumeração de $\{(x, \mathfrak{C}): x \in I \backslash\{\underline{0}\}$, $\left.\mathfrak{C} \in[\mathcal{A}]^{\leq \omega}\right\}$ tal que para cada $(x, \mathfrak{C})$, o conjunto $\left\{\alpha:\left(x_{\alpha}, \mathfrak{C}_{\alpha}\right)=(x, \mathfrak{C})\right.$ e $\left.\alpha \notin \bigcup \mathfrak{C}\right\}$ tem cardinalidade $\mathfrak{c}$. O item $(\mathrm{v})$ será satisfeito se fizermos $d_{x_{\alpha}}(\alpha)=1$ para todo $\alpha \in[\omega, \mathfrak{c})$.

A construção de $D$. Construiremos $D \uparrow \alpha=\left\{d_{x} \uparrow \alpha: x \in I\right\}$ por indução em $\alpha \in[\omega, \mathfrak{c})$. Ao final, faremos $d_{x}=d_{x} \uparrow \mathfrak{c}$ e $D=\left\{d_{x}: x \in I\right\}$. Em cada estágio, uma vez que $D \uparrow \alpha$ é determinado tomaremos $y_{\alpha} \in 2^{\alpha}$, ponto de acumulação de $\left\{d_{x} \uparrow \alpha: x \in E_{\alpha}\right\}$ e faremos, como prometido, $B_{\alpha}^{i}=y_{\alpha} \cup A_{\alpha}^{i} \uparrow[\alpha, \mathfrak{c}), \forall i \in 2$.

Nossas hipóteses de indução são:

(0) $\forall x \in I, d_{x} \uparrow \omega=x$;

(1) $\forall x, y \in I, d_{x}\left\lceil\alpha+d_{y} \uparrow \alpha=d_{x+y}\left\lceil\alpha\right.\right.$ e $\forall x \in I d_{x}\left\lceil\beta \subseteq d_{x}\lceil\alpha\right.$;

(2) se $\omega \leq \beta<\alpha$, então $d_{x_{\beta}}(\beta)=1$;

(3) se $\omega \leq \beta<\alpha$, então $B_{\beta}^{0}\left\lceil\alpha\right.$ e $B_{\beta}^{1}\left\lceil\alpha\right.$ são pontos de acumulação de $\left\{d_{x} \uparrow \alpha: x \in E_{\beta}\right\}$.

Se $\alpha=\omega$, então $d_{x} \uparrow \omega=x(x \in I)$ e $D \uparrow \omega=\left\{d_{x} \uparrow \omega: x \in I\right\}$. Se $\alpha>\omega$ é ordinal limite, então faremos $d_{x} \uparrow \alpha=\bigcup_{\omega \leq \beta<\alpha} d_{x} \uparrow \beta$ para todo $x \in I$. Verifica-se facilmente que valem os itens (1) e (2) para este caso. Para o item (3), tome $F \in[\alpha]^{<\omega}$ e $\beta \in \alpha$ quaisquer e $\gamma \in \mathfrak{c}$ tal que $F \subseteq \gamma$ e $\beta<\gamma$. Pelo item (3) aplicado a $\gamma$, existe $x_{i} \in E_{\beta}$ tal que $d_{x_{i}} \uparrow F=B_{\beta}^{i} \uparrow F$ e $d_{x_{i}} \uparrow \gamma \neq B_{\beta}^{i} \uparrow \gamma, \forall i \in 2$. Logo, $d_{x_{i}} \uparrow F=B_{\beta}^{i} \uparrow F$ e $d_{x_{i}} \uparrow \alpha \neq B_{\beta}^{i} \uparrow \alpha$, $\forall i \in 2$, e está provado o item (3).

Considere agora o caso $\alpha \Longrightarrow \alpha+1$. Temos que definir $d_{x}(\alpha)$ para todo $x \in I$. O item (1) diz que a função $\phi: x \longmapsto d_{x}(\alpha)$ deve ser um homomorfismo de $I$ em 2. O item (2) diz que devemos ter $\phi\left(x_{\alpha}\right)=1$. Para o item (3) necessitamos que $\forall \beta \in[\omega, \alpha], F \in[\alpha+1]^{<\omega}$ e $i \in 2$, o conjunto $\left\{x \in E_{\beta}: d_{x} \uparrow F=B_{\beta}^{i} \uparrow F\right\}$ seja infinito. Se $\alpha \notin F$ isto é satisfeito, pois $B_{\beta}^{i} \uparrow F$ é ponto de acumulação de $\left\{d_{x} \uparrow \alpha: x \in E_{\beta}\right\}$, por hipótese de indução. Dessa forma, seja $F \in[\alpha]^{<\omega}$ e considere $F \cup\{\alpha\}$. Então, $d_{x} \uparrow(F \cup\{\alpha\})=B_{\beta}^{i} \uparrow(F \cup\{\alpha\})$ é equivalente a $d_{x} \uparrow F=B_{\beta}^{i} \uparrow F$ e $\phi(x)=B_{\beta}^{i}(\alpha)$ de forma que a seguinte expressão deve ser satisfeita na 
construção de $D$ :

$(*)$ se $\beta \in[\omega, \alpha], F \in[\alpha+1]^{<\omega}$ e $i \in 2$, então o conjunto $\left\{x \in E_{\beta}: d_{x} \uparrow F=B_{\beta}^{i} \uparrow F\right.$ e $\left.\phi(x)=B_{\beta}^{i}(\alpha)\right\}$ é infinito.

Obteremos esta expressão através de $\mathbf{M} \mathbf{A}_{\mathrm{e}}$ aplicado ao conjunto enumerável parcialmente ordenado $\mathbf{P}=\{p \in F n(I, 2): \operatorname{dom}(p)$ é subgrupo finito de $I, p$ é homomorfismo, $x_{\alpha} \in \operatorname{dom}(p)$ e $\left.p\left(x_{\alpha}\right)=1\right\}$, ordenado pela inclusão inversa. Para todo $x \in I, A_{x}=\{p: x \in \operatorname{dom}(p)\}$ é denso em $\mathbf{P}:$ se $x \notin \operatorname{dom}(p)$, então podemos tomar $p^{\prime} \leq p$ tal que $\operatorname{dom}\left(p^{\prime}\right)=\langle\operatorname{dom}(p) \cup\{x\}\rangle$ e $p^{\prime}(x)=0$. Assim, se $\mathcal{G}$ é filtro em $\mathbf{P}$ que intersecta todo $A_{x}$, então $\phi=\bigcup \mathcal{G}$ é um homomorfismo em $I$ que satisfaz $\phi\left(x_{\alpha}\right)=1$. Para termos $(*)$, precisaremos de mais conjuntos densos: $\forall \beta \in[\omega, \alpha], F \in[\alpha]^{<\omega}, i \in 2$ e $n \in \omega$ faça $A(\beta, F, i, n)=\left\{p: \mid\left\{x \in \operatorname{dom}(p): x \in E_{\beta} ; d_{x} \uparrow F=B_{\beta}^{i} \uparrow F\right.\right.$ e $\left.\left.p(x)=B_{\beta}^{i}(\alpha)\right\} \mid \geq n\right\}$. Temos que $A(\beta, F, i, 0)=\mathbf{P}$ e que se $p \in A(\beta, F, i, n)$, então existe $p^{\prime} \leq p$ tal que $p^{\prime} \in A(\beta, F, i, n+1)$. De fato, se $p \in A(\beta, F, i, n)$ então como $\left\{x \in E_{\beta} ; d_{x} \uparrow F=B_{\beta}^{i} \uparrow F\right\}$ é infinito, podemos tomar $x$ neste conjunto $\operatorname{com} x \notin \operatorname{dom}(p)$ e $p^{\prime} \leq p$ tal que $\operatorname{dom}\left(p^{\prime}\right)=$ $\langle\operatorname{dom}(p) \cup\{x\}\rangle$. Mas daí, $p^{\prime} \in A(\beta, F, i, n+1) \subseteq A(\beta, F, i, n)$ e teremos que cada $A(\beta, F, i, n)$ é denso, como queríamos. Como a cardinalidade dos conjuntos densos é $|\alpha|<\mathfrak{c}$, podemos aplicar $\mathbf{M} \mathbf{A}_{\mathrm{e}}$ a $\mathbf{P}$, obtendo um filtro $\mathcal{G}$ em $\mathbf{P}$ que intersecta cada $A_{x}$ e cada $A(\beta, F, i, n)$. Então, $\phi=\bigcup \mathcal{G}$ é um homomorfismo e satisfaz (*). Isto completa a construção de $D$.

Exemplo 2.2.1: Existem dois subgrupos enumeravelmente compactos $H_{0}$ e $H_{1}$ de $2^{\mathfrak{c}}$ tais que $H_{0} \times H_{1}$ não é enumeravelmente compacto.

Demonstração: Se tivermos $D, G_{0}$ e $G_{1}$ como descrito pelos itens acima então faremos $H_{0}=D+G_{0}$ e $H_{1}=D+G_{1}$.

(a) $H_{0}$ e $H_{1}$ são enumeravelmente compactos. Seja $i \in\{0,1\}$ e seja $I=\left\{d_{n}+g_{n}: n \in \omega\right\} \subseteq H_{i}$ infinito. Se $\left\{d_{n}: n \in \omega\right\}$ é finito, então do fato de que $G_{i}$ 
é $\omega$-limitado segue-se que $C l_{H_{i}} I$ é compacto, de forma que $I$ possui ponto de acumulação em $H_{i}$. Se $\left\{d_{n}: n \in \omega\right\}$ é infinito, seja $g \in G_{i}$ ponto de acumulação. Tome $p \in \omega^{*}$ tal que $g=p-\lim \left\{d_{n}: n \in \omega\right\}$. Então como $G_{i}$ é $\omega$-limitado temos que $\overline{\left\{g_{n}: n \in \omega\right\}}$ é compacto em $G_{i}$ e, portanto, $\left\{g_{n}: n \in \omega\right\}$ possui p-limite $h \in G_{i}$. Como o p-limite da soma termo a termo de sequências é igual a soma dos p-limites temos que $g+h \in G_{i}$ é ponto de acumulação de $I$ em $H_{i}$.

(b) $H_{0} \times H_{1}$ não é enumeravelmente compacto. Pelo item (iii), temos que $D \cap\left(G_{0}+G_{1}\right)=\{\underline{0}\}$ e $G_{0} \cap G_{1}=\{\underline{0}\}$ de forma que $H_{0} \cap H_{1}=D+G_{0} \cap D+G_{1}=D$. Com efeito, se $d_{0}+g_{0}=d_{1}+g_{1} \in D+G_{0} \cap D+G_{1}$, então $d_{0}-d_{1}=g_{1}-g_{0} \in D \cap\left(G_{0}+G_{1}\right)=\{\underline{0}\}$. Daí, $g_{1}=g_{0} \in G_{0} \cap G_{1}=\{\underline{0}\}$ donde $d_{0}+g_{0}=d_{0} \in D$. Assim, a diagonal $\left\{(x, x): x \in 2^{\mathfrak{c}}\right\}$ restrita ao subespaço $H_{0} \times H_{1}$ é fechada neste subespaço e é igual a $\{(d, d): d \in D\}$, conjunto enumerável. Supondo, por contradição, que $H_{0} \times H_{1}$ seja enumeravelmente compacto teremos que $\{(d, d): d \in D\}$ será enumeravelmente compacto, o que entraria em contradição com o lema 1.1.8.

\subsection{Segundo exemplo de Hart e van Mill}

O próximo exemplo encontra-se originalmente também em [6]. Aqui construiremos um grupo enumeravelmente compacto $H$ tal que $H^{2}$ não é enumeravelmente compacto. A construção é basicamente a mesma da secção anterior, mas com uma notação mais "carregada". Descreveremos agora como deverá ser esse grupo.

Para um conjunto de ordinais $x$ façamos $\bar{x}=x \times 2$ e considere a função $\bar{\rho}: \overline{\mathfrak{c}} \longmapsto \overline{\mathfrak{c}}$ definida por $\bar{\rho}(\alpha, i)=(\alpha, 1-i)$. Esta induz um automorfismo $\rho$ de $2^{\overline{\mathfrak{c}}}$. Veremos que $H$ pode ser considerado um subgrupo de $2^{\mathfrak{c}} \times 2^{\mathfrak{c}}$ contendo $H_{0} \times\{\underline{0}\}$ e $\{\underline{0}\} \times H_{1}$, em que $H_{0}$ e $H_{1}$ são 
parecidos com os da construção anterior. A notação ficará mais "enxuta"se trabalharmos em $2^{\overline{\mathfrak{c}}}$ em vez de $2^{\mathfrak{c}} \times 2^{\mathfrak{c}}$. Desta vez $H$ será $D+G_{0}+G_{1}+G_{2}$ e $H_{i}$ será $D+G_{i}, \forall i \in 2$, com $D$ subgrupo de $2^{\overline{\mathfrak{c}}}$ enumerável e $G_{0}, G_{1}$ e $G_{2} \operatorname{subgrupos} \omega$-limitados de $2^{\overline{\mathfrak{c}}}$. Para $i \in 2$, faça $K_{i}=\left\{x \in 2^{\overline{\mathfrak{c}}}: \operatorname{supp}(x) \subseteq \mathfrak{c} \times\{i\}\right\}$. Chame $D_{i}=D \cap K_{i}, \forall i \in 2$. Faremos $D=D_{0}+D_{1}$ e $D_{1}=\rho\left(D_{0}\right)$. Além disso, teremos $G_{i} \subseteq K_{i}$ e $H_{i} \subseteq K_{i}, \forall i \in 2$. Da mesma forma que na construção anterior, cada $E \subseteq D$ infinito terá ponto de acumulação em $G_{0}+G_{1}+G_{2}$ fazendo com que $H=D+G_{0}+G_{1}+G_{2}$ seja enumeravelmente compacto. Teremos também $G_{1} \cap \rho\left(G_{0}\right)=\{\underline{0}\}$ e $D \cap\left(G_{0}+G_{1}\right)=\{\underline{0}\}$ e isso, como veremos adiante, implicará que $H_{0} \times H_{1}$ não é enumeravelmente compacto. Como $H_{0}$ e $H_{1}$ são fechados em $H$, teremos que $H_{0} \times H_{1}$ será fechado em $H^{2}$ e, portanto, $H^{2}$ não poderá ser enumeravelmente compacto. Usaremos $G_{i}$ para conter os pontos de acumulação de $D_{i}, \forall i \in 2$, e $G_{2}$ para conter os pontos de acumulação dos conjuntos infinitos de $D \backslash\left(D_{0} \cup D_{1}\right)$.

Lema 2.3.1 $\left(\mathbf{M A}_{\mathrm{e}}\right)$ : Existem 4 subgrupos $D, G_{0}, G_{1}$ e $G_{2}$ de $2^{\overline{\mathfrak{c}}}$ que satisfazem:

(i) $D$ é enumerável e $D_{1}=\rho\left(D_{0}\right)$;

(ii) $G_{0}, G_{1}$ e $G_{2}$ são $\omega$-limitados, com $G_{i} \subseteq K_{i}, \forall i \in 2$;

(iii) $G_{1} \cap \rho\left(G_{0}\right)=\{\underline{0}\}=D \cap\left(G_{0}+G_{1}+G_{2}\right)$;

(iv) se $E \subseteq D$ é infinito, então $E$ possui pontos de acumulação em $D+G_{0}+G_{1}+G_{2}$.

$\mathbf{M A}_{\mathrm{e}}$ é usado novamente somente na construção de $D$.

Demonstração: Seja $\mathcal{A}$ família $\omega$-independente em c. Partiremos $\mathcal{A}$ em 4 famílias $\omega$-independentes disjuntas de tamanho $\mathfrak{c}, \mathcal{A}_{0}, \mathcal{A}_{1}, \mathcal{A}_{2}$ e $\mathcal{A}_{3}$. Definimos $\mathcal{A}_{0}^{+}=\left\{A \times\{0\}: A \in \mathcal{A}_{0}\right\}, \mathcal{A}_{1}^{+}=\left\{A \times\{1\}: A \in \mathcal{A}_{1}\right\}$ e $\mathcal{A}_{i}^{+}=\left\{A \times 2: A \in \mathcal{A}_{i}\right\}$, $i=2$, 3. Como na construção anterior, indentificamos cada $A \in \mathcal{A}_{0}^{+} \cup \mathcal{A}_{1}^{+} \cup \mathcal{A}_{2}^{+} \cup \mathcal{A}_{3}^{+}$com sua função característica em $2^{\overline{\mathfrak{c}}}$.

Seja $I=\left\{x \in 2^{\bar{\omega}}: \operatorname{supp}(x)\right.$ é finito $\}$ e $\forall i \in 2$ seja $I_{i}=\{x \in I: \operatorname{supp}(x) \subseteq \omega \times\{i\}\}$. Vê-se que $I=I_{0}+I_{1}$ e que cada $x \in I$ é escrito de forma única como $x_{0}+x_{1}$, com $x_{0} \in I_{0}$ 
e $x_{1} \in I_{1}$. Assim, faremos $D=\left\{d_{x}: x \in I\right\}$ e $D_{i}=\left\{d_{x}: x \in I_{i}\right\}, \forall i \in 2$, na construção abaixo.

Para ver para quais subconjuntos infinitos $E \subseteq D$ nós teremos que prover pontos de acumulação, considere $E \subseteq I$ infinito. Poderemos ter 3 opções mais simples: (i) $E \cap I_{0}$ é infinito, o que fará com que $\left\{d_{x}: x \in E \cap I_{0}\right\}$ tenha ponto de acumulação em $G_{0}$, na construção abaixo; (ii) $E \cap I_{1}$ é infinito, o que fará com que $\left\{d_{x}: x \in E \cap I_{1}\right\}$ tenha ponto de acumulação em $G_{1}$; (iii) para um número infinito de $x \in E, x$ é simétrico $(x=\rho(x)$ ), e neste caso $G_{2}$ conterá o ponto de acumulação. Se nenhum dos casos acima acontecer, então sem perda de generalidade $\forall x \in E, x_{0}, x_{1}, x+\rho(x) \neq \underline{0}$. Ainda neste caso, poderemos ter um subconjunto infinito $E^{\prime} \subseteq E$ e um $a \in I$ tal que (iv) $\forall x \in E^{\prime}, x_{0}=a$; (v) $\forall x \in E^{\prime}$, $x_{1}=a$ ou (vi) $\forall x \in E^{\prime}, x+\rho(x)=a$. Nos casos (iv) ou (v), $d_{a}+g$ será ponto de acumulação de $\left\{d_{x}: x \in E^{\prime}\right\}$, onde $g$ é ponto de acumulação de $\left\{d_{x}+d_{a}: x \in E^{\prime}\right\}$. De fato, tome como exemplo o caso $x_{0}=a, \forall x \in E^{\prime}$. Temos que $\forall x \in E^{\prime} d_{x}=d_{x_{0}}+d_{x_{1}}=d_{a}+d_{x_{1}}$. Somando $d_{a}$ a ambos os lados da equação temos que $d_{x}+d_{a}=d_{x_{1}}, \forall x \in E^{\prime}$. Pelo caso (ii) acima, $\exists g \in G_{1}$ ponto de acumulação de $\left\{d_{x}+d_{a}: x \in E^{\prime}\right\}$. Segue-se $d_{a}+g$ será ponto de acumulação de $\left\{d_{x}: x \in E^{\prime}\right\}$. No caso (vi), tome $E^{\prime \prime} \subseteq E^{\prime}$ infinito e $b \in I$ tal que $\forall x \in E^{\prime \prime}$, $x \cap(x+\rho(x))=b$. Então, $x=b+(x \cap \rho(x)), \forall x \in E^{\prime \prime}$, e $d_{b}+g$ é ponto de acumulação de $\left\{d_{x}: x \in E^{\prime \prime}\right\}$ onde $g \in G_{2}$ é ponto de acumulação de $\left\{x \cap \rho(x): x \in E^{\prime \prime}\right\}$ (do caso (iii)).

Finalmente, se (iv), (v) e (vi) não ocorrerem obteremos um subconjunto infinito $E^{\prime} \subseteq E$ tal que

$(*) \forall x, y \in E^{\prime} x \neq y \Longrightarrow x_{0} \neq y_{0}, x_{1} \neq y_{1}$ e $x+\rho(x) \neq y+\rho(y)$.

Concluímos que será suficiente prover pontos de acumulação para $\left\{d_{x}: x \in E\right\}$ nos casos $E \subseteq I_{0}, E \subseteq I_{1}$, cada $x \in E$ é simétrico e $E$ satisfaz $(*)$. Enumeraremos todos os conjuntos importantes para a construção de $D$ :

$$
-\left\{A_{\alpha}^{i}: \omega \leq \alpha<\mathfrak{c}\right\} \text {, enumeração de } \mathcal{A}_{i}^{+} \text {sem repetições }(i \in 4),
$$


$-\left\{E_{\alpha}^{i}: \omega \leq \alpha<\mathfrak{c}\right\}$, enumeração de $\left[I_{i}\right]^{\omega}(i \in 2)$,

$-\left\{E_{\alpha}^{2}: \omega \leq \alpha<\mathfrak{c}\right\}$, enumeração de $\left\{E \in[I]^{\omega}: E\right.$ é simétrico $\}$,

$-\left\{E_{\alpha}^{3}: \omega \leq \alpha<\mathfrak{c}\right\}$, enumeração de $\left\{E \in[I]^{\omega}: E \operatorname{satisfaz}(*)\right\}$,

$-\left\{\left(x_{\alpha}, \mathfrak{C}_{\alpha}\right): \omega \leq \alpha<\mathfrak{c}\right\}$, enumeração de $(I \backslash\{\underline{0}\}) \times[\mathcal{A}] \leq \omega$ de forma que para cada $(x, \mathfrak{C})$ o conjunto $\left\{\alpha:\left(x_{\alpha}, \mathfrak{C}_{\alpha}\right)=(x, \mathfrak{C})\right.$ e $\left.\alpha \notin \bigcup \mathfrak{C}\right\}$ tem cardinalidade $\mathfrak{c}\left(D \cap\left(G_{0}+G_{1}+G_{2}\right)=\{\underline{0}\}\right)$.

Vamos agora à construção de $D$. Por indução em $\alpha \in[\omega, \mathfrak{c}]$, construímos $D \uparrow \bar{\alpha}=\left\{d_{x} \uparrow \bar{\alpha}: x \in I\right\}$ satisfazendo:

(0) $\forall x \in I, d_{x} \uparrow \bar{\omega}=x$; se $\omega \leq \beta<\alpha$, então $d_{x} \uparrow \bar{\beta} \subseteq d_{x} \uparrow \bar{\alpha}$; e $\forall x, y \in I$, $d_{x} \uparrow \bar{\alpha}+d_{y} \uparrow \bar{\alpha}=d_{x+y} \uparrow \bar{\alpha}$

(1) $\forall x \in I_{i}, \operatorname{supp}\left(d_{x}\right) \cap \bar{\alpha} \subseteq \alpha \times\{i\}, \forall i \in 2$;

(2) $\forall x \in I, d_{\rho(x)} \uparrow \bar{\alpha}=\rho\left(d_{x} \uparrow \bar{\alpha}\right)$;

(3) se $\omega \leq \beta<\alpha$, então $d_{x_{\beta}}(\beta, 0)=1$ se $x_{\beta} \notin I_{1}$ ou $d_{x_{\beta}}(\beta, 1)=1$ se $x_{\beta} \notin I_{0}$.

O item (1) será responsável por garantir que $D_{i}=\left\{d_{x}: x \in I_{i}\right\}, \forall i \in 2$, e o item (0) que $D$ seja subgrupo de $2^{\overline{\mathfrak{c}}}$. O item (2) será responsável por fazer $\rho\left(D_{0}\right)=D_{1}$. Já o item $(3)$, como na construção anterior, será responsável por cuidar que $D \cap\left(G_{0}+G_{1}+G_{2}\right)=\{\underline{0}\}$. Uma vez que tenhamos $D \uparrow \bar{\alpha}$, podemos tomar para $i=0,1,2,3$ um ponto de acumulação $x_{\alpha}^{i}$ para $\left\{d_{x}\left\lceil\bar{\alpha}: x \in E_{\alpha}^{i}\right\}\right.$ em $2^{\overline{\mathfrak{c}}}$. Daí, para $i \in 4$ podemos fazer $B_{\alpha}^{i}=x_{\alpha}^{i} \cup A_{\alpha}^{i} \uparrow \overline{[\alpha, \mathfrak{c})}$. Note que $B_{\alpha}^{i} \in K_{i}, \forall i \in 2$, uma vez que $x_{\alpha}^{i}$ é ponto de acumulação de uma sequência em $K_{i}$ e $A_{\alpha}^{i} \in \mathcal{A}_{i}^{+}=\left\{A \times\{i\}: A \in \mathcal{A}_{i}\right\} \subseteq K_{i}$. Queremos também que para $i=0,1,2,3$ tenhamos

(4) se $\omega \leq \beta<\alpha$, então $B_{\beta}^{i} \uparrow \bar{\alpha}$ é ponto de acumulação de $\left\{d_{x} \uparrow \bar{\alpha}: x \in E_{\beta}^{i}\right\}, \forall i \in 4$.

Vamos à construção por indução propriamente dita:

Para o caso $\alpha=\omega$, faça $d_{x} \uparrow \bar{\omega}=x, x \in I$, e $D \uparrow \bar{\omega}=I$. Temos que os itens (3) e (4) valem de forma vazia e os itens (0), (1) e (2) são prontamente verificados com 
$x$ no lugar de $d_{x}$. Se $\alpha$ é ordinal limite, faça $d_{x} \uparrow \bar{\alpha}=\bigcup_{\omega \leq \beta<\alpha} d_{x} \uparrow \bar{\beta}, \forall x \in I$. Para a igualdade $d_{x} \uparrow \bar{\alpha}+d_{y} \uparrow \bar{\alpha}=d_{x+y} \uparrow \bar{\alpha}$ do item (0), por exemplo, tome $\gamma<\alpha$. Teremos que $d_{x}(\gamma, i)+d_{y}(\gamma, i)=d_{x+y}(\gamma, i)$, pois o item $(0)$ vale para $d_{x} \uparrow \bar{\beta} \operatorname{com} \gamma<\beta<\alpha$. Daí, $\forall \gamma<\alpha$ e $i \in 2$ temos que vale a igualdade anterior e, portanto, $d_{x} \uparrow \bar{\alpha}+d_{y} \uparrow \bar{\alpha}=d_{x+y} \uparrow \bar{\alpha}$. Para os outros itens vale raciocínio semelhante.

Agora, considere o passo sucessor $\alpha \Longrightarrow \alpha+1$. Como na construção anterior, faremos uso de um homomorfismo $\phi$ para definir $d_{x}(\alpha, 0)$ e $d_{x}(\alpha, 1)$ para todo $x \in I$. Dessa vez o homorfismo terá a forma $\phi: I \longmapsto 2^{2}$. Queremos que

$$
\begin{aligned}
& \phi(\rho(x))(0)=\phi(x)(1) \text { e } \phi(\rho(x))(1)=\phi(x)(0) \text { para que valha o item }(2), \\
& \phi(x)(1)=0\left(x \in I_{0}\right) \text { e } \phi(x)(0)=0\left(x \in I_{1}\right) \text { para o item }(1) \\
& \phi\left(x_{\alpha}\right)(0)=1 \text { se } x_{\alpha} \notin I_{1} \text { e } \phi\left(x_{\alpha}\right)(1)=1 \text { se } x_{\alpha} \notin I_{0} \text { para o item (3). }
\end{aligned}
$$

Usamos então o conjunto parcialmente ordenado enumerável:

$\mathbf{P}=\left\{p \in F n\left(I, 2^{2}\right): \operatorname{dom}(p)\right.$ é subgrupo finito de $I, p$ é homomorfismo, se $x \in \operatorname{dom}(p)$, então $x_{0}, x_{1}, \rho(x) \in \operatorname{dom}(p), p\left(x_{0}\right)(1)=p\left(x_{1}\right)(0)=0$ e $p(\rho(x))(i)=p(x)(1-i)(i \in 2)$; $x_{\alpha} \in \operatorname{dom}(p)$ e $\left.x_{\alpha} \notin I_{0} \Longrightarrow p\left(x_{\alpha}\right)(1)=1, x_{\alpha} \notin I_{1} \Longrightarrow p\left(x_{\alpha}\right)(0)=1\right\}$, ordenado pela inclusão inversa.

Seja $x \in I$ e $\mathcal{A}_{x}=\{p \in \mathbf{P}: x \in d o m(p)\}$. Para mostrar que $\mathcal{A}_{x}$ é denso, considere o grupo $F_{x}$ gerado por $\left\{x_{0}, x_{1}, \rho\left(x_{0}\right), \rho\left(x_{1}\right)\right\}$. Temos que encontrar $p^{\prime} \leq p$ com $\operatorname{dom}\left(p^{\prime}\right)=\operatorname{dom}(p)+F_{x}$, pois na definição de $\mathbf{P}$ exigimos que $(* *)$ se $x \in \operatorname{dom}(p)$, então $x_{0}, x_{1}, \rho(x) \in \operatorname{dom}(p)$. Considerando que $x \notin \operatorname{dom}(p)$, as exigências sobre o $\operatorname{dom}(p)$ feitas na definição de $\mathbf{P}$ implicam em 4 possibilidades - (I), (II), (III) e (IV) abaixo, para $F_{x} \cap \operatorname{dom}(p)$.

(I) Podemos ter que $F_{x} \cap \operatorname{dom}(p)=\{\underline{0}\}$, e neste caso podemos fazer $p^{\prime}\left(x_{i}\right)(0)=$ $p^{\prime}\left(x_{i}\right)(1)=0, \forall i \in 2$, que $p^{\prime}(x)=p^{\prime}\left(x_{0}\right)+p^{\prime}\left(x_{1}\right)$ e $p^{\prime}(\rho(x))=\rho\left(p^{\prime}(x)\right)$ estarão também 
determinados.

Se considerarmos a possibilidade $x_{0} \in F_{x} \cap \operatorname{dom}(p)$, então teremos que $\rho\left(x_{0}\right) \in F_{x} \cap \operatorname{dom}(p)$ e $x_{0}+\rho\left(x_{0}\right) \in F_{x} \cap \operatorname{dom}(p)$, por $(* *)$. Perceba que $\left\{\underline{0}, x_{0}, \rho\left(x_{0}\right), x_{0}+\rho\left(x_{0}\right)\right\}$ forma um subgrupo. Perceba também que $x_{1} \notin F_{x} \cap \operatorname{dom}(p)$, pois caso contrário $x_{0}+x_{1}=x$ estaria em $F_{x} \cap \operatorname{dom}(p)$. Também não podemos ter $\rho\left(x_{1}\right) \in F_{x} \cap \operatorname{dom}(p)$, pois do contrário teríamos $\rho\left(\rho\left(x_{1}\right)\right)=x_{1} \in F_{x} \cap \operatorname{dom}(p)$. Temos também que $x_{1}+\rho\left(x_{1}\right) \in F_{x} \cap \operatorname{dom}(p) \Longrightarrow x_{0}+x_{1}+\rho\left(x_{1}\right) \in F_{x} \cap \operatorname{dom}(p) \Longrightarrow$ $x+\rho\left(x_{1}\right) \in F_{x} \cap \operatorname{dom}(p) \Longrightarrow x_{1} \in F_{x} \cap \operatorname{dom}(p)$, por $(* *)$. Usando raciocínio semelhante podemos excluir todas as outras possibilidades de elementos de $F_{x}$ que possam estar também em $\operatorname{dom}(p)$, quando $x_{0} \in \operatorname{dom}(p)$ e $x \notin \operatorname{dom}(p)$. Daí, se $x_{0} \in F_{x} \cap \operatorname{dom}(p)$ teremos obrigatoriamente que:

(II) $F_{x} \cap \operatorname{dom}(p)=\left\{\underline{0}, x_{0}, \rho\left(x_{0}\right), x_{0}+\rho\left(x_{0}\right)\right\}$, e neste caso podemos fazer $p^{\prime}\left(x_{1}\right)(0)=$ $p^{\prime}\left(x_{1}\right)(1)=0$, que $p^{\prime}(x)=p^{\prime}\left(x_{0}\right)+p^{\prime}\left(x_{1}\right)$ e $p^{\prime}(\rho(x))=\rho\left(p^{\prime}(x)\right)$ estarão também determinados.

Com um raciocínio análogo ao anterior temos o

(III) $F_{x} \cap \operatorname{dom}(p)=\left\{\underline{0}, x_{1}, \rho\left(x_{1}\right), x_{1}+\rho\left(x_{1}\right)\right\}$, e neste caso podemos fazer $p^{\prime}\left(x_{0}\right)(0)=$ $p^{\prime}\left(x_{0}\right)(1)=0$.

Finalmente, se $x_{0}+\rho\left(x_{1}\right) \in F_{x} \cap \operatorname{dom}(p)$, então $\rho\left(x_{0}+\rho\left(x_{1}\right)\right)=\rho\left(x_{0}\right)+x_{1} \in F_{x} \cap \operatorname{dom}(p)$ e $x_{0}+\rho\left(x_{1}\right)+\rho\left(x_{0}\right)+x_{1}=x+\rho(x) \in F_{x} \cap \operatorname{dom}(p)$. Vemos facilmente que $\left\{\underline{0}, x_{0}+\rho\left(x_{1}\right)\right.$, $\left.x_{1}+\rho\left(x_{0}\right), x+\rho(x)\right\}$ forma um subgrupo. Usando dos mesmos raciocínios para o caso (ii) vemos que estes são os únicos elementos de $F_{x}$ que podem estar em $\operatorname{dom}(p)$, uma vez que $x_{0}+\rho\left(x_{1}\right)$ pertença a $F_{x} \cap \operatorname{dom}(p)$ e $x \notin \operatorname{dom}(p)$. Daí,

(IV) $F_{x} \cap \operatorname{dom}(p)=\left\{\underline{0}, x_{0}+\rho\left(x_{1}\right), x_{1}+\rho\left(x_{0}\right), x+\rho(x)\right\}$, e neste caso $\varepsilon=p(x+\rho(x))$ é simétrico, já que vale $p(\rho(x))(i)=p(x)(1-i), \forall i \in 2$. Temos que $\varepsilon(0)=\varepsilon(1)=j$. Podemos fazer $p^{\prime}\left(x_{0}\right)(0)=j, p^{\prime}\left(x_{0}\right)(1)=0$ e $p^{\prime}\left(x_{1}\right)(0)=0$. Temos que $p^{\prime}\left(x_{1}\right)(1)=p^{\prime}\left(\rho\left(x_{1}\right)\right)(0)=$ 
$p^{\prime}\left(x_{0}+\rho\left(x_{1}\right)\right)(0)+p^{\prime}\left(x_{0}\right)(0)$.

Assim, se $\mathcal{G}$ é filtro em $\mathbf{P}$ que intersecta todos os $\mathcal{A}_{x}$, então $\phi=\bigcup \mathcal{G}$ é homomorfismo que satisfaz os itens $(0),(1),(2)$ e (3). Para o item (4), devemos atender à condição:

se $\beta \in[\omega, \alpha], F \in[\overline{\alpha+1}]^{<\omega}$ e $i \in 4$, então o conjunto $\left\{x \in E_{\beta}^{i}: d_{x} \uparrow F=B_{\beta}^{i} \uparrow F\right\}$ é infinito.

Se $(\alpha, 0) \notin F$ e $(\alpha, 1) \notin F$, isto é satisfeito, pois $B_{\beta}^{i} \uparrow F$ é ponto de acumulação de $\left\{d_{x} \uparrow \alpha: x \in E_{\beta}\right\}$, por hipótese de indução. Dessa forma, seja $F \in[\bar{\alpha}]^{<\omega}$ e considere $F \cup\{\bar{\alpha}\}$. Então, $d_{x} \uparrow(F \cup\{\bar{\alpha}\})=B_{\beta}^{i} \uparrow(F \cup\{\bar{\alpha}\})$ é equivalente a $d_{x} \uparrow F=B_{\beta}^{i} \uparrow F \mathrm{e}$ $\phi(x)(j)=B_{\beta}^{i}(\alpha, j), \forall j \in 2$. Considere os seguintes conjuntos: se $\beta \in[\omega, \alpha], F \in[\bar{\alpha}]^{<\omega}$, $i \in 4$ e $n \in \omega$ seja $A(\beta, F, i, n)$ o conjunto

$\left\{p:\left|\left\{x \in \operatorname{dom}(p): x \in E_{\beta}^{i}, d_{x} \uparrow F=B_{\beta}^{i} \uparrow F, \phi(x)(j)=B_{\beta}^{i}(\alpha, j), \forall j \in 2\right\}\right| \geq n\right\}$.

Então, $A(\beta, F, i, 0)=\mathbf{P}$ e se $p \in A(\beta, F, i, n)$, então existe $p^{\prime} \leq p$ com $p^{\prime} \in A(\beta, F, i, n+1):$

$i=0,1$ : Tome $x \in E_{\beta}^{i} \operatorname{com} d_{x} \uparrow F=B_{\beta}^{i} \uparrow F$ e $x \notin \operatorname{dom}(p)$. Isto é possível, pois o conjunto $\left\{x \in E_{\beta}: d_{x} \uparrow F=B_{\beta}^{i} \uparrow F\right\}$ é infinito e $\operatorname{dom}(p)$ é finito. Então, $F_{x}=\left\langle\left\{x_{0}, x_{1}, \rho\left(x_{0}\right), \rho\left(x_{1}\right)\right\}\right\rangle=\{\underline{0}, x, \rho(x), x+\rho(x)\}$ e $F_{x} \cap \operatorname{dom}(p)=\{\underline{0}\}$. Defina $p^{\prime} \leq p$ por $\operatorname{dom}\left(p^{\prime}\right)=\operatorname{dom}(p)+F_{x}$ e $p^{\prime}(x)(j)=B_{\beta}^{i}(\alpha, j), \forall j \in 2$. Note que $p^{\prime}(\rho(x))$ e $p^{\prime}(x+\rho(x))$ estarão definidos a partir de $p^{\prime}(x)$ e que $B_{\beta}^{i}(\alpha, 1-i)=0$, de forma que a definição está de acordo.

$i=2$ : Tome $x \in E_{\beta}^{2} \operatorname{com} d_{x} \uparrow F=B_{\beta}^{2} \uparrow F$ e $x \notin \operatorname{dom}(p)$. Então, $F_{x}=$ $\left\langle\left\{x_{0}, x_{1}, \rho\left(x_{0}\right), \rho\left(x_{1}\right)\right\}\right\rangle=\left\{\underline{0}, x_{0}, x_{1}, x\right\}$ e $F_{x} \cap \operatorname{dom}(p)=\{\underline{0}\}$. Note que $B_{\beta}^{2}(\alpha, 0)=B_{\beta}^{2}(\alpha, 1)$ uma vez que $\alpha \geq \beta$ e $B_{\beta}^{2}=x_{\beta}^{2} \cup A_{\beta}^{2} \uparrow \overline{[\beta, \mathfrak{c})}$, com $A_{\beta}^{2} \in \mathcal{A}_{2}^{+}=\left\{A \times 2: A \in \mathcal{A}_{2}\right\}$. Assim, podemos definir $p^{\prime} \leq p \operatorname{com} \operatorname{dom}\left(p^{\prime}\right)=\operatorname{dom}(p)+F_{x}, p^{\prime}\left(x_{0}\right)(0)=p^{\prime}\left(x_{1}\right)(1)=B_{\beta}^{2}(\alpha, 0) \mathrm{e}$ $p^{\prime}\left(x_{0}\right)(1)=p^{\prime}\left(x_{1}\right)(0)=0$. 
$i=3$ : Tome $x \in E_{\beta}^{3} \operatorname{com} d_{x} \uparrow F=B_{\beta}^{3} \uparrow F$ e $x_{0}, x_{1}, x_{0}+\rho\left(x_{1}\right) \notin \operatorname{dom}(p)$. Novamente $F_{x} \cap \operatorname{dom}(p)=\{\underline{0}\}$. Como $B_{\beta}^{3}(\alpha, 0)=B_{\beta}^{3}(\alpha, 1)$, podemos novamente definir $p^{\prime} \leq p$ tal que $\operatorname{dom}\left(p^{\prime}\right)=\operatorname{dom}(p)+F_{x}$ e $p^{\prime}\left(x_{0}\right)(0)=p^{\prime}\left(x_{1}\right)(1)=B_{\beta}^{3}(\alpha, 0)$ e $p^{\prime}\left(x_{0}\right)(1)=p^{\prime}\left(x_{1}\right)(0)=0$.

Ao final da indução teremos $d_{x}=d_{x} \uparrow \overline{\mathfrak{c}}, \forall x \in I$, e $D=D \uparrow \overline{\mathfrak{c}}$. Faremos $G_{0}$ o subgrupo $\omega$-limitado gerado por $\mathcal{B}_{0}$ e similarmente $G_{1}$ gerado por $\mathcal{B}_{1}$ e $G_{2}$ gerado por $\mathcal{B}_{2} \cup \mathcal{B}_{3}$. Faremos $H_{0}=D_{0}+G_{0}, H_{1}=D_{1}+G_{1}$ e $H=D+G_{0}+G_{1}+G_{2}$. Pelo item (3), sabemos que para cada família enumerável $\mathcal{A}^{\prime} \subseteq \mathcal{A}$ e para todo $d \in D$ teremos que $\left|\operatorname{supp}(d) \backslash \bigcup\left(\mathcal{A}^{\prime} \times 2\right)\right|=\mathfrak{c}$. Da mesma forma que na construção anterior, isto implica em $D \cap\left(G_{0}+G_{1}+G_{2}\right)=\{\underline{0}\}$. O item (4) assegura que todo subconjunto infinito de $D$ terá ponto de acumulação em $H$.

Exemplo 2.3.1: Existe um subgrupo enumeravelmente compacto $H$ de $2^{\mathfrak{c}} \times 2^{\mathfrak{c}}$ tal que $H^{2}$ não é enumeravelmente compacto.

Considere $D, G_{0}, G_{1}, G_{2}, H_{0}, H_{1}$ e $H$ como descrito pelos itens acima.

(a) $H$ é enumeravelmente compacto. Seja $I=\left\{d_{n}+g_{n}: n \in \omega\right\} \subseteq H$ infinito, com $d_{n} \in D$ e $g_{n} \in G_{0}+G_{1}+G_{2}$. Se $\left\{d_{n}: n \in \omega\right\}$ é finito, então como $G_{0}+G_{1}+G_{2}$ é $\omega$-limitado temos que $C l_{H} I$ é compacto em $G_{0}+G_{1}+G_{2}$, de forma que $I$ possui ponto de acumulação em $H$. Se $\left\{d_{n}: n \in \omega\right\}$ é infinito, seja $h \in G_{0}+G_{1}+G_{2}$ ponto de acumulação. Tome $p \in \omega^{*}$ tal que $h=p-\lim \left\{d_{n}: n \in \omega\right\}$. Então, como $G_{0}+G_{1}+G_{2}$ é $\omega$-limitado temos que $\overline{\left\{g_{n}: n \in \omega\right\}}$ é compacto em $G_{0}+G_{1}+G_{2}$ e, portanto, $\left\{g_{n}: n \in \omega\right\}$ possui p-limite $g \in G_{0}+G_{1}+G_{2}$. Como o p-limite da soma termo a termo de sequências é igual a soma dos p-limites temos que $g+h \in G_{0}+G_{1}+G_{2}$ é ponto de acumulação de $I$ em $H$.

(b) $H^{2}$ não é enumeravelmente compacto. Primeiramente, temos que $H_{0} \times H_{1}$ é fechado em $H^{2}$. De fato, sabemos que $H_{0}$ e $H_{1}$ são fechados em $H$, já que se $x \in H \backslash H_{i}$, teremos que $\operatorname{supp}(x) \cap(\mathfrak{c} \times\{1-i\}) \neq \emptyset$ e daí, podemos tomar um aberto na topologia de Tychonoff em $H \backslash H_{i}$ que contém $x, \forall i \in 2$. Logo, supondo por contradição que $H^{2}$ é 
enumeravelmente compacto teremos que $H_{0} \times H_{1}$ será também enumeravelmente compacto. Provaremos, então, que $\left\{(d, \rho(d)): d \in D_{0}\right\}=\{(h, \rho(h)): h \in H\} \cap H_{0} \times H_{1}$. Como a diagonal $\{(h, \rho(h)): h \in H\}$ é também fechada, teremos que o conjunto enumerável $\left\{(d, \rho(d)): d \in D_{0}\right\}$ será fechado de $H^{2}$ e, portanto, enumeravelmente compacto, o que é contradição pelo lema 1.1.8. Basta provar então a inclusão $H_{0} \times H_{1} \subseteq\left\{(d, \rho(d)): d \in D_{0}\right\}$. Tome $(h, \rho(h)) \in H_{0} \times H_{1}$. Podemos representar esse elemento por $\left(d_{0}+g_{0}, \rho\left(d_{0}\right)+\rho\left(g_{0}\right)\right)$. Está claro que podemos obter a inclusão provando que $g_{0}=\underline{0}$. Por um lado, temos que $\rho\left(g_{0}\right) \in \rho\left(H_{0}\right)=D_{1}+\rho\left(G_{0}\right) \subseteq D+\rho\left(G_{0}\right)$. Por outro lado, temos também que $\rho\left(g_{0}\right) \in H_{1}=D_{1}+G_{1} \subseteq D+G_{1}$. Daí, $\rho\left(g_{0}\right) \in\left(D+\rho\left(G_{0}\right)\right) \cap\left(D+G_{1}\right) \subseteq D$. Logo, $g_{0} \in D$, donde $g_{0} \in D \cap G_{0}=\{\underline{0}\}$ e obtemos o resultado. 


\section{Capítulo 3}

\section{Grupo enumeravelmente compacto cujo quadrado não é a partir de um grupo de van Douwen.}

\subsection{Preliminares}

Primeiramente, vamos discutir um pouco a notação usada neste capítulo.

Dado um grupo abeliano $G$ e $g \in G$, a ordem de $g$ é o menor $n \in \omega$ tal que $n g=0$ e é denotada por $o(g)$. Para todo $n \in \omega$, denotamos por $\mathbb{Z}_{n}$ o grupo cíclico de ordem $n$ $\{\overline{0}, \overline{1}, \ldots, \overline{n-1}\}$. Dado um conjunto de ordinais $I$ e $h \in \mathbb{Z}_{n}{ }^{I}$, definimos o suporte de $h$, $\operatorname{supp}(h)$, por $\{\alpha \in I: h(\alpha) \neq 0\}$. Denotaremos por $\mathbb{Z}_{n}{ }^{(I)}$ o conjunto de todas as funções $h \in \mathbb{Z}_{n}{ }^{I}$ que têm suporte finito.

Sejam $F$ e $I$ conjuntos de ordinais, $n \in \omega$ e $\kappa$ um cardinal qualquer. Dada uma família $\left\{x_{\xi}: \xi \in F\right\} \subseteq \mathbb{Z}_{n}{ }^{\kappa}$ e $f \in\left(\mathbb{Z}_{n}{ }^{(I)}\right)^{\omega} \operatorname{com} \operatorname{supp}(f(n)) \subseteq F, \forall n \in \omega$, definimos 
$x_{f(n)}=\sum_{\lambda \in \operatorname{supp}(f(n))} f(n)(\lambda) x_{\lambda}, \forall n \in \omega$.

Demonstraremos agora alguns lemas técnicos que permitirão a construção do grupo de van Douwen (grupo abeliano, infinito, enumeravelmente compacto, sem sequências nãotriviais convergentes) na secção 3.2 e serão usados também no capítulo 4. Usaremos as propriedades de ultrafiltros seletivos incomparáveis discutidas na secção 1.2.

Lema 3.1.1: Seja $p$ um ultrafiltro seletivo e $\left\{a_{k}: k \in \omega\right\} \in p$ uma sequência estritamente crescente tal que $k<a_{k}$ para cada $k \in \omega$. Então, existe $I \subseteq \omega$ tal que

i) $\left\{a_{k}: k \in I\right\} \in p$;

ii) $\left\{\left[k, a_{k}\right]: k \in I\right\}$ são intervalos disjuntos de $\omega$.

Demonstração: Seja $A=\left\{a_{i}: i \in \omega\right\} \in p$ e defina uma partição $\left\{P_{0}, P_{1}\right\}$ de $[\omega]^{2}$ como segue: para cada $\{b, c\} \in[\omega]^{2},\{b, c\}$ é elemento de $P_{0}$ se, e somente se, existem $i, k \in \omega$ com $i<k$ tal que $\{b, c\}=\left\{a_{i}, a_{k}\right\}$ e $a_{i}<k$. Pela propriedade de Ramsey para $p$, existe $B \in p$ tal que $B \subseteq A$ e ou $[B]^{2} \subseteq P_{0}$ ou $[B]^{2} \subseteq P_{1}$. Afirmamos que $[B]^{2} \nsubseteq P_{1}$. De fato, seja $\left\{t_{n}: n \in \omega\right\}$ sequência crescente tal que $B=\left\{a_{t_{n}}: n \in \omega\right\}$. Então, $\left\{a_{t_{0}}, a_{t_{n}}\right\} \in P_{1}$ implica em $a_{t_{0}} \geq t_{n}, \forall n>0$, o que é contradição. Portanto, $[B]^{2} \subseteq P_{0}$. Assim, se $\left\{a_{i}, a_{k}\right\} \in[B]^{2}$ com $a_{i}<a_{k}$, então $i<a_{i}<k<a_{k}$. Seja $I \subseteq \omega$ tal que $B=\left\{a_{k}: k \in I\right\}$. Então, as condições i) e ii) são satisfeitas.

Lema 3.1.2: Sejam $p_{0}$ e $p_{1}$ ultrafiltros seletivos incomparáveis. Seja $\left\{a_{k}^{j}: k \in \omega\right\} \in p_{j}$, $j<2$, uma sequência crescente tal que $k<a_{k}^{j}$, para todo $k \in \omega$ e $j<2$. Então, existem $I_{0}$ e $I_{1}$, subconjuntos de $\omega$, tais que:

i) $\left\{a_{k}^{j}: k \in I_{j}\right\} \in p_{j}, \forall j<2$;

ii) $\left\{\left[k, a_{k}^{j}\right]: j<2\right.$ e $\left.k \in I_{j}\right\}$ são intervalos 2 a 2 disjuntos de $\omega$. 
Demonstração: Usando o lema anterior, obtemos subconjuntos $J_{0}$ e $J_{1}$ de $\omega$ que satisfazem:

1) $B_{j}=\left\{a_{k}^{j}: k \in J_{j}\right\} \in p_{j}, \forall j<2$;

2) $\left\{\left[k, a_{k}^{j}\right]: k \in J_{j}\right\}$ são intervalos 2 a 2 disjuntos de $\omega, \forall j<2$.

Uma vez que $p_{0}$ e $p_{1}$ são ultrafiltros distintos, podemos assumir que 3) $B_{0} \cap B_{1}=\emptyset$. De fato, se tomarmos $C \in p_{0} \backslash p_{1}$, digamos, podemos trabalhar com os conjuntos $B_{0} \cap C \in p_{0}$ e $B_{1} \cap(\omega \backslash C) \in p_{1}$.

Refinaremos, então, $J_{0}$ e $J_{1}$ para $I_{0}$ e $I_{1}$ de forma que os itens i) e ii) sejam satisfeitos. Por causa da propriedade 2), o item ii) será satisfeito se vale a seguinte propriedade:

4) $\left[i, a_{i}^{0}\right] \cap\left[k, a_{k}^{1}\right]=\emptyset, \forall i \in I_{0}, k \in I_{1}$.

Usando a sequência estritamente crescente $B_{0}=\left\{a_{k}^{0}: k \in I_{0}\right\}$ e o item 2), obtemos uma partição de $\omega:\left\{\left[0, a_{1}^{0}\right),\left[a_{k}^{0}, a_{k+1}^{0}\right): k \in I_{0}\right\}$. Como cada intervalo da partição é finito, pela seletividade de $p_{1}$, existe $C \in p_{1}$ tal que $C$ intercepta cada intervalo em somente um ponto. Podemos tomar $C_{1}=C \cap B_{1} \in p_{1}$. Do item 3), temos que as extremidades dos intervalos da partição não podem estar em $C_{1}$. Assim, temos que 5) existe $C_{1} \in p_{1}$ tal que $C_{1} \subseteq B_{1}$ e se $a, b$ são elementos consecutivos de $B_{0}$, então $\left|[a, b] \cap C_{1}\right| \leq 1$.

Seja $T$ o conjunto $\bigcup\left\{\left[i, a_{i}^{0}\right]: a_{i}^{0} \in B_{0}\right.$ e $\left.\left[i, a_{i}^{0}\right] \cap B_{1} \neq \emptyset\right\}$. Defina $f_{0}: \omega \longmapsto \omega$ como se segue:

6a) $f_{0} \uparrow \omega \backslash T$ é arbitrário;

6b) se $n \in T$, então existem um único $t \in \omega$ tal que $n \in\left[t, a_{t}^{0}\right]$ e um único $m \in \omega$ tal que $\{m\}=\left[t, a_{t}^{0}\right] \cap C_{1}$.

Defina $f_{0}(n)=m$.

Uma vez que $\beta f_{0}\left(p_{0}\right) \neq p_{1}$ ( $p_{0}$ e $p_{1}$ são ultrafiltros incomparáveis), existe $D_{1}$ tal que

7) $D_{1} \in p_{1}, D_{1} \subseteq C_{1}$ e $f_{0}^{-1}\left(D_{1}\right) \notin p_{0}$.

Seja $C_{0}=B_{0} \backslash f_{0}^{-1}\left(D_{1}\right) \in p_{0}$. Afirmamos que

8) se $a_{i}^{0} \in C_{0}$, então $\left[i, a_{i}^{0}\right] \cap D_{1}=\emptyset$. De fato, se $a_{t}^{1} \in\left[i, a_{i}^{0}\right] \cap D_{1}$, então 
$\left\{a_{t}^{1}\right\}=\left[i, a_{i}^{0}\right] \cap D_{1}=\left[i, a_{i}^{0}\right] \cap C_{1}$. Portanto, $f_{0}\left(a_{i}^{0}\right)=a_{t}^{1} \in D_{1}$, pelo item $\left.6 \mathrm{~b}\right)$. Entretanto, $a_{i}^{0} \in f_{0}^{-1}\left(D_{1}\right)$ contradiz a definição de $C_{0}$. Usando a seletividade de $p_{0}$, podemos encontrar $D_{0}$ tal que

9) $D_{0} \subseteq C_{0}, D_{0} \in p_{0}$ e se $a, b \in D_{1}$ são elementos consecutivos de $D_{1}$, então $\left|[a, b] \cap D_{0}\right| \leq 1$.

A partir de um argumento similar ao já usado, existem $E_{0}, E_{1}$ tais que 10) $E_{j} \subseteq D_{j}$ e $E_{j} \in p_{j}, \forall j \in 2$, e 11) se $a_{t}^{1} \in E_{1}$, então $\left[t, a_{t}^{1}\right] \cap E_{0}=\emptyset$.

Do item 8) e do fato que $E_{0} \subseteq C_{0}$ e $E_{1} \subseteq D_{0}$, concluímos que

12) se $a_{i}^{0} \in E_{0}$, então $\left[i, a_{i}^{0}\right] \cap E_{1}=\emptyset$.

Seja $I_{j} \subseteq \omega$ tal que $E_{j}=\left\{a_{k}^{j}: k \in I_{j}\right\}, \forall j<2$.

Do item 10), segue-se que $I_{0}$ e $I_{1}$ satisfazem o item i).

Como observado anteriormente, para mostrar que vale o item ii) é suficiente mostrar que vale o item 4). Para prová-lo, suponha por contradição que existam $i \in I_{0}$ e $t \in I_{1}$ tais que $\left[i, a_{i}^{0}\right] \cap\left[t, a_{t}^{1}\right] \neq \emptyset$. Então, ou $a_{i}^{0} \in\left[t, a_{t}^{i}\right]$ ou $a_{t}^{1} \in\left[i, a_{i}^{0}\right]$. Mas isso iria de encontro aos itens 11) ou 12). Logo, $I_{0}$ e $I_{1}$ satisfazem o item ii).

Lema 3.1.3: Seja $\left\{p_{j}: j \in \omega\right\}$ uma família de ultrafiltros seletivos incomparáveis. Seja $\left\{a_{k}^{j}: k \in \omega\right\} \in p_{j}$ uma sequência crescente tal que $k<a_{k}^{j}, \forall k, j \in \omega$. Então, existe uma família $\left\{I_{j}: j \in \omega\right\}$ de subconjuntos de $\omega$ tal que:

i) $\left\{a_{k}^{j}: k \in I_{j}\right\} \in p_{j}, \forall j \in \omega$;

ii) $\left\{\left[k, a_{k}^{j}\right]: j \in \omega\right.$ e $\left.k \in I_{j}\right\}$ são intervalos 2 a 2 disjuntos de $\omega$.

Demonstração: Por indução, podemos construir um subconjunto $I_{j, t}$ de $\omega, \forall j, t \in \omega$ tal que:

a) $I_{j, t+1} \subseteq I_{j, t}, \forall t \in \omega$

b) $\left\{\left[k, a_{k}^{j}\right]: j \leq t\right.$ e $\left.k \in I_{j, t}\right\}$ são intervalos 2 a 2 disjuntos de $\omega, \forall t \in \omega$; 
c) $\left\{a_{k}^{j}: k \in I_{j, t}\right\} \in p_{j}, \forall j, t \in \omega$.

Do lema anterior, existe $I_{0,0} \subseteq \omega$ tal que $\left\{a_{k}^{0}: k \in I_{0,0}\right\} \in p_{0}$ e $\left\{\left[k, a_{k}^{j}\right]: k \in I_{0,0}\right\}$ são 2 a 2 disjuntos. Assim, as condições a)-c) são satisfeitas para $j, t \leq 0$. Suponha que o conjunto $I_{j, t}$ tenha sido definido para todo $j, t \leq s$, satisfazendo as condições a)-c). Seja $I_{s+1,0}=\omega \mathrm{e}$ aplicando o lema anterior $s$ vezes, defina por indução $I_{s+1, t+1}$ e $I_{t, s+1}$ para cada $t \leq s$ de tal forma que:

1) $I_{t, s+1} \subseteq I_{t, s}$ e $I_{s+1, t+1} \subseteq I_{s+1, t}, \forall t \leq s$

2) $\left\{\left[k, a_{k}^{t}\right]: k \in I_{t, s+1}\right\} \cup\left\{\left[k, a_{k}^{s+1}\right]: k \in I_{s+1, t+1}\right\}$ são intervalos 2 a 2 disjuntos de $\omega$;

3) $\left\{a_{k}^{t}: k \in I_{t, s+1}\right\} \in p_{t}$ e $\left\{a_{k}^{s+1}: k \in I_{s+1, t+1}\right\} \in p_{s+1}, \forall t \leq s$.

Temos que a)-c) são satisfeitos para cada $j, t \leq s+1$. Assim, $I_{j, t}$ pode ser definido para cada $j, t \in \omega$.

Para todo $j \in \omega, p_{j}$ é P-ponto. Pelo item c), existe $K_{j} \subseteq \omega$ tal que:

d) $K_{j} \subseteq I_{j, j}$ e $K_{j} \backslash I_{j, t}$ é finito para cada $t \in \omega$;

e) $\left\{a_{k}^{j}: k \in K_{j}\right\} \in p_{j}$.

Pelo item d), existe $M_{j} \in \omega$ tal que:

f) $K_{l} \subseteq I_{l, j} \cup M_{j}, \forall l \leq j$;

Defina g) $I_{j}=K_{j} \backslash \max \left\{a_{k}^{l}+1: l<j \wedge k<M_{j}\right\}$. O conjunto $K_{j} \backslash I_{j}$ é finito. Pelo item e), o conjunto $I_{j}$ satisfaz i) para cada $j \in \omega$.

Afirmamos que os $I_{j}^{\prime} s$ também satisfazem o item ii). Sejam $j, j^{\prime}, k, k^{\prime} \in \omega$ com $k \in I_{j}$ e $k^{\prime} \in I_{j^{\prime}}$. Se $j=j^{\prime}$, então dos itens d) e g) segue-se que $k, k^{\prime} \in I_{j} \subseteq I_{j, j}$. Pelos itens a) e $\mathrm{b})$, temos que $\left[k, a_{k}^{j}\right] \cap\left[k^{\prime}, a_{k^{\prime}}^{j}\right]=\emptyset$. Se $k^{\prime} \notin I_{j^{\prime}, j}$, então, pelo item $\left.\mathrm{f}\right), k^{\prime} \in M_{j}$. Do item g), segue-se que $k>a_{k^{\prime}}^{j^{\prime}}$. Portanto, $\left[k, a_{k}^{j}\right] \cap\left[k^{\prime}, a_{k^{\prime}}^{j^{\prime}}\right]=\emptyset$ e vale o item ii).

\subsection{Construção do grupo de van Douwen}


Nesta secção, construiremos um grupo de van Douwen, isto é, um grupo abeliano, infinito, enumeravelmente compacto e sem sequências não-triviais convergentes a partir de um ultrafiltro seletivo. Esta contrução pode ser encontrada em [5]. O nosso grupo de van Douwen $G$ será gerado por um subconjunto linearmente independente de $\{0,1\}^{\mathfrak{c}}$. Vamos primeiramente a um lema auxiliar:

Lema 3.2.1: Seja $p \in \omega^{*}$ um ultrafiltro seletivo. Então, existe uma família de funções injetoras $\left\{f_{\xi}: \xi<\mathfrak{c}\right\} \subseteq\left([\mathfrak{c}]^{<\omega}\right)^{\omega}$ tais que:

1) $\bigcup_{n<\omega} f_{\xi}(n) \subseteq \max \{\omega, \xi\}, \forall \xi<\mathfrak{c}$

2) $\left\{\left[f_{\xi}\right]_{p}: \xi<\mathfrak{c}\right\} \cup\left\{[\vec{\beta}]_{p}: \beta<\mathfrak{c}\right\}$ é base para $\left([\mathfrak{c}]^{<\omega}\right)^{\omega} / p$;

3) para cada função injetora $g \in\left([\mathfrak{c}]^{<\omega}\right)^{\omega}$, existem $\varsigma_{0}, \varsigma_{1}<\mathfrak{c}$ distintos e duas sequências crescentes de inteiros positivos $\left(n_{k}^{0}\right)_{k<\omega}$ e $\left(n_{k}^{1}\right)_{k<\omega}$ tais que $f_{\varsigma_{i}}(k)=g\left(n_{k}^{i}\right), \forall k<\omega$ e $i \in\{0,1\}$.

Demonstração: Seja $\left\{g_{\xi}: \xi<\mathfrak{c}\right\}$ uma enumeração de todas as funções injetoras de $\left([\mathfrak{c}]^{<\omega}\right)^{\omega}$ de tal forma que cada função é listada duas vezes e $\bigcup_{n<\omega} g_{\xi}(n) \subseteq \max \{\omega, \xi\}, \forall \xi<\mathfrak{c}$. Cuidemos primeiramente desta enumeração: Sabemos que $\left|\left([\mathfrak{c}]^{<\omega}\right)^{\omega}\right|=\mathfrak{c}$. Considere então $A=\left\{h \in\left([\mathfrak{c}]^{<\omega}\right)^{\omega}: h\right.$ é injetora $\}$ e $\left\{h_{\xi}: \xi<\mathfrak{c}\right\}$ uma enumeração dos elementos de $A$ de tal forma que dada $h \in A,\left|\left\{\xi: h_{\xi}=h\right\}\right|=\mathfrak{c}$. Seja agora $I: \omega \longmapsto[\mathfrak{c}]^{<\omega}$ tal que $I(n)=\{n\}, \forall n \in \omega$. Considere $\xi<\mathfrak{c}$. Se $\bigcup_{n \in \omega} \operatorname{supp}\left(h_{\xi}(n)\right) \subseteq \max \{\omega, \xi\}$, defina $g_{\xi}=h_{\xi}$. Se $\bigcup_{n \in \omega} \operatorname{supp}\left(h_{\xi}(n)\right) \nsubseteq \xi$, então defina $g_{\xi}=I$ de forma que $\bigcup_{n \in \omega} \operatorname{supp}\left(g_{\xi}(n)\right) \subseteq \max \{\omega, \xi\}$, $\forall \xi<\mathfrak{c}$. Vamos garantir agora que $A \subseteq\left\{g_{\xi}: \xi<\mathfrak{c}\right\}$. Se $h \in A$, então $\exists \gamma<\mathfrak{c}$ tal que $\bigcup_{n \in \omega} \operatorname{supp}(h(n)) \subseteq \gamma$. Como $\left|\left\{\xi: h_{\xi}=h\right\}\right|=\mathfrak{c}$, existe $\xi>\gamma$ tal que $h_{\xi}=h$, logo $\bigcup_{n \in \omega} \operatorname{supp}\left(h_{\xi}(n)\right) \subseteq \gamma \subseteq \xi$. Assim, $h=h_{\xi}=g_{\xi}$ está na enumeração.

Usaremos indução transfinita para provar o lema. Considere $\alpha<\mathfrak{c}$ e suponha que, para cada $\xi<\alpha$, nós tenhamos definido uma função injetora $f_{\xi}: \omega \longmapsto[\mathfrak{c}]^{<\omega}$ tal que:

i) Para cada $m<\omega$, existe $n<\omega$ tal que $f_{\xi}(m)=g_{\xi}(n), \forall \xi<\alpha$; 
ii) $\left\{\left[f_{\varsigma}\right]_{p}: \varsigma<\xi\right\} \cup\left\{[\vec{\beta}]_{p}: \beta<\mathfrak{c}\right\}$ é linearmente independente, $\forall \xi<\alpha$;

iii) se $\left\{\left[f_{\varsigma}\right]_{p}: \varsigma<\xi\right\} \cup\left\{\left[g_{\xi}\right]_{p}\right\} \cup\left\{[\vec{\beta}]_{p}: \beta<\mathfrak{c}\right\}$ é linearmente independente, então $f_{\xi}=g_{\xi}$, $\forall \xi<\alpha$.

Se $\left\{\left[f_{\xi}\right]_{p}: \xi<\alpha\right\} \cup\left\{\left[g_{\alpha}\right]_{p}\right\} \cup\left\{[\vec{\beta}]_{p}: \beta<\mathfrak{c}\right\}$ é linearmente independente, então definimos $f_{\alpha}=g_{\alpha}$. Vamos assumir que $\left\{\left[f_{\xi}\right]_{p}: \xi<\alpha\right\} \cup\left\{\left[g_{\alpha}\right]_{p}\right\} \cup\left\{[\vec{\beta}]_{p}: \beta<\mathfrak{c}\right\}$ não seja linearmente independente. Seja $\left\{A_{\mu}: \mu<\mathfrak{c}\right\}$ uma família quase-disjunta de subconjuntos infinitos de $\omega$. Para cada $\mu<\mathfrak{c}$, seja $h_{\mu}: \omega \longmapsto A_{\mu}$ uma bijeção. Então, definimos $h_{\alpha, \mu}: \omega \longmapsto[\mathfrak{c}]^{<\omega}$ por $h_{\alpha, \mu}(n)=g_{\alpha}\left(h_{\mu}(n)\right), \forall n<\omega$. É evidente que $\left\{n \in \omega: h_{\alpha, \mu}(n)=h_{\alpha, v}(n)\right\}$ é finito para $\mu<v<\mathfrak{c}$. Portanto, $\left\{\left[h_{\alpha, \mu}\right]_{p}: \mu<\mathfrak{c}\right\}$ é família de funções 2 a 2 distintas. Logo, podemos encontrar $\mu_{\alpha}<\mathfrak{c}$ tal que $\left[h_{\alpha, \mu_{\alpha}}\right]_{p} \notin\left\langle\left\{\left[f_{\varsigma}\right]_{p}: \varsigma<\xi\right\} \cup\left\{[\vec{\beta}]_{p}: \beta<\max \{\omega, \alpha\}\right\}\right\rangle$, por argumento de cardinalidade. Faça $f_{\alpha}=h_{\alpha, \mu_{\alpha}}$. Claramente os itens i) e iii) são satisfeitos também para $\alpha$. Sabemos que $\left\{\left[f_{\xi}\right]_{p}: \xi \leq \alpha\right\} \cup\left\{[\vec{\beta}]_{p}: \beta<\max \{\omega, \alpha\}\right\}$ é linearmente independente. Uma vez que $\bigcup_{n<\omega} f_{\xi}(n) \subseteq \max \{\omega, \xi\}, \forall \xi \leq \alpha$, temos também que $\left\{\left[f_{\xi}\right]_{p}: \xi \leq \alpha\right\} \cup\left\{[\vec{\beta}]_{p}: \beta<\mathfrak{c}\right\}$ é linearmente independente. Isto mostra o item ii). Afirmamos que a família $\left\{f_{\xi}: \xi<\mathfrak{c}\right\}$ satisfaz todas as condições. De fato, pela construção acima, $\left\{\left[f_{\xi}\right]_{p}: \xi<\mathfrak{c}\right\} \cup\left\{[\vec{\beta}]_{p}: \beta<\mathfrak{c}\right\}$ é base para $\left([\mathfrak{c}]^{<\omega}\right)^{\omega} / p$, pois é l.i. e no processo de indução verificamos todas as funções injetoras $\left\{g_{\xi}: \xi<\mathfrak{c}\right\}$ que poderiam entrar em nossa enumeração (lema 1.3.2). Vamos provar que vale o item 3). Tome $\varsigma_{0}, \varsigma_{1}<\mathfrak{c}$ distintos tais que $\varsigma_{0}<\varsigma_{1}$ e $g=g_{\varsigma_{0}}=g_{\varsigma_{1}}$. Do item i), podemos tomar duas sequências crescentes de inteiros positivos $\left(n_{k}^{0}\right)_{k<\omega}$ e $\left(n_{k}^{1}\right)_{k<\omega}$ tais que $f_{\varsigma_{i}}(k)=g\left(n_{k}^{i}\right), \forall k<\omega$ e $i \in\{0,1\}$.

Para o restante do capítulo 3, considere uma família $\left\{f_{\xi}: \xi<\mathfrak{c}\right\} \subseteq\left([\mathfrak{c}]^{<\omega}\right)^{\omega}$ que satisfaça as 3 propriedades listadas no lema anterior e uma enumeração $\left\{F_{\alpha}: \alpha<\mathfrak{c}\right\}$ de $[\mathfrak{c}]^{<\omega} \backslash\{\emptyset\}$.

Lema 3.2.2: Seja $p \in \omega^{*}$ um ultrafiltro seletivo. Suponha que para cada $\alpha<\mathfrak{c}$ tenhamos um homomorfismo não-trivial $\phi_{\alpha}:[\mathfrak{c}]^{<\omega} \longmapsto\{0,1\}$ tal que 
i) $\phi_{\alpha}(\{\xi\})=p-\lim \left\{\phi_{\alpha}\left(f_{\xi}(n)\right): n \in \omega\right\}, \forall \xi<\mathfrak{c}$;

ii) $\phi_{\alpha}\left(F_{\alpha}\right)=1$.

Para $\xi<\mathfrak{c}$, definimos $x_{\xi} \in\{0,1\}^{\mathfrak{c}}$ por $x_{\xi}(\alpha)=\phi_{\alpha}(\{\xi\}), \forall \alpha<\mathfrak{c}$. Então, o conjunto $X=\left\{x_{\xi}: \xi<\mathfrak{c}\right\}$ é linearmente independente em $\{0,1\}^{\mathfrak{c}}$ e $G=\langle X\rangle$ é grupo p-compacto sem sequências não-triviais convergentes, ou seja, é grupo de van Douwen.

Demonstração: Seja $\left\{\xi_{0}, \ldots, \xi_{k}\right\} \in[\mathfrak{c}]^{<\omega}$. Tome $\alpha<\mathfrak{c}$ tal que $F_{\alpha}=\left\{\xi_{0}, \ldots, \xi_{k}\right\}$. Então, pelo item ii),

$$
\left(x_{\xi_{0}}+\ldots+x_{\xi_{k}}\right)(\alpha)=\phi_{\alpha}\left(\left\{\xi_{0}\right\}\right)+\ldots+\phi_{\alpha}\left(\left\{\xi_{k}\right\}\right)=\phi_{\alpha}\left(F_{\alpha}\right)=1
$$

Isto mostra que $\left\{x_{\xi}: \xi<\mathfrak{c}\right\}$ é linearmente independente em $\left.\{0,1\}\right\}^{\mathfrak{c}}$. Agora mostraremos que $G$ é p-compacto. Antes disso, note que devido ao item i) temos que $x_{\xi}=p-\lim \left\{\sum_{\mu \in f_{\xi}(n)} x_{\mu}: n \in \omega\right\}=p-\lim \left\{x_{f_{\xi}(n)}: n \in \omega\right\}, \forall \xi<\mathfrak{c}$. Seja $\left(a_{n}\right)_{n<\omega}$ uma sequência 1-1 qualquer em $G$. Tome $h \in\left([\mathfrak{c}]^{<\omega}\right)^{\omega}$ injetora tal que $a_{n}=x_{h(n)}, \forall n \in \omega$. Como $\left\{\left[f_{\xi}\right]_{p}: \xi<\mathfrak{c}\right\} \cup\left\{[\vec{\beta}]_{p}: \beta<\mathfrak{c}\right\}$ é base para $\left([\mathfrak{c}]^{<\omega}\right)^{\omega} / p$, existem $\xi_{0}, \ldots, \xi_{k} \in \mathfrak{c}$ e $E \in[\mathfrak{c}]^{<\omega}$ tais que $[h]_{p}=\left(\triangle_{i \leq k}\left[f_{\xi_{i}}\right]_{p}\right) \triangle\left(\triangle_{\mu \in E}[\vec{\mu}]_{p}\right)$. Portanto, podemos encontrar $B \in p$ tal que $B \subseteq A$ e $h(n)=\left(\triangle_{i \leq k} f_{\xi_{i}}(n)\right) \triangle E, \forall n \in B$. Segue-se, então, que $x_{h(n)}=\sum_{i \leq k} x_{f_{\xi_{i}}(n)}+x_{E}, \forall n \in B$. Assim, $\sum_{i \leq k} x_{\xi_{i}}+x_{E}=p-\lim \left\{x_{h(n)}: n \in \omega\right\}$ e isto mostra que $G$ é p-compacto.

Vamos provar agora que nenhuma sequência não-trivial em $G$ converge. Seja $\left(y_{n}\right)_{n<\omega}$ uma sequência não-trivial em $G$ e tome uma função injetora em $\left([\mathfrak{c}]^{<\omega}\right)^{\omega}$ tal que $y_{n}=x_{g(n)}$. Pelo item 3) do lema anterior, existem $\varsigma_{0}, \varsigma_{1}<\mathfrak{c}$ distintos e duas sequências crescentes de inteiros positivos $\left(n_{k}^{0}\right)_{k<\omega}$ e $\left(n_{k}^{1}\right)_{k<\omega}$ tais que $f_{\varsigma_{i}}(k)=g\left(n_{k}^{i}\right), \forall k<\omega$ e $i \in\{0,1\}$. Uma vez que $x_{\varsigma_{i}}=p-\lim \left\{x_{f_{\varsigma_{i}}(k)}: k \in \omega\right\}=p-\lim \left\{x_{g\left(n_{k}^{i}\right)}: k \in \omega\right\}=p-\lim \left\{y_{n_{k}^{i}}: k \in \omega\right\}, \forall i \in 2$, teremos que $x_{\varsigma_{0}}$ e $x_{\varsigma_{1}}$ são ambos pontos de acumulação de $\left\{y_{n}: n \in \omega\right\}$.

Para finalizar a construção do grupo de van Douwen basta provar a existência do 
homomorfismo $\phi_{\alpha}:[\mathfrak{c}]^{<\omega} \longmapsto\{0,1\}, \forall \alpha<\mathfrak{c}$, satisfazendo os itens i) e ii) do lema anterior. Para isso, precisaremos de dois lemas; o segundo, responsável pela construção do homomorfismo propriamente dito.

Lema 3.2.3: Seja $p \in \omega^{*}$ um ultrafiltro seletivo. Para cada $E_{0} \in[\mathfrak{c}]^{<\omega} \backslash\{\emptyset\}$, existem $\left\{b_{i}: i \in \omega\right\} \in p$ e $\left\{E_{i}: 0<i<\omega\right\} \subseteq[\mathfrak{c}]^{<\omega}$ tais que

1) $\omega \subseteq \bigcup_{i<\omega} E_{i}$

2) $E_{i} \cup \bigcup_{\xi \in E_{i}} f_{\xi}\left(b_{i}\right) \subseteq E_{i+1}, \forall i \in \omega$

3) $\left\{f_{\xi}\left(b_{i}\right): \xi \in E_{i}\right\} \cup\left\{\{\mu\}: \mu \in E_{i}\right\}$ é linearmente independente, $\forall i \in \omega$.

Demonstração: Faça $F_{0}=E_{0}$ e seja $F_{n+1}=n \cup F_{n} \cup \bigcup_{\xi \in F_{n}} \bigcup_{m \leq n} f_{\xi}(m)$, para todo $1 \leq n \leq \omega$. Como $\left\{\left[f_{\xi}\right]_{p}: \xi<\mathfrak{c}\right\} \cup\left\{[\vec{\beta}]_{p}: \beta<\mathfrak{c}\right\}$ é linearmente independente, temos que $A_{n}=\left\{k \in \omega:\left\{f_{\xi}(k): \xi \in F_{n}\right\} \cup\left\{\{\mu\}: \mu \in F_{n}\right\}\right.$ é linearmente independente $\} \in p, \forall n<\omega$.

Ultrafiltros seletivos são P-pontos, logo existe $A \in p$ tal que $A \backslash A_{n}$ é finito, $\forall n \in \omega$.

Seja $h_{j}: \omega \longmapsto \omega$ função crescente tal que

4) $A \backslash A_{n} \subseteq h(n)$.

Temos que $h$ define uma partição em $\omega:[0, h(1)],\{[h(n)+1, h(n+1)]: n \geq 1\}$. Pela seletividade de $p$, existe $C \in p$ tal que $\left|\left[0, h_{j}(1)\right] \cap C\right|=1$ e $|[h(n)+1, h(n+1)] \cap C|=1$, $\forall n \geq 1$. Tome $B=(C \cap A) \backslash([0, h(1)] \cap C)$. Então,

5) $B \cap h(1)=\emptyset, B \subseteq A, B \in p$ e $|[h(n)+1, h(n+1)] \cap B| \leq 1, \forall n \in \omega$.

Seja $\left\{a_{n}: n \in \omega\right\}$ enumeração crescente de $B$. De (5), segue-se que $a_{n}>h(n), \forall n \in \omega$. Assim, de (4) temos:

6) $n<a_{n}$ e $a_{n} \in A_{n}, \forall n \in \omega$.

O item 6) afirma que a sequência $\left\{a_{n}: n \in \omega\right\}$ satisfaz as hipóteses do lema 3.1.1. Logo, existe $I \subseteq \omega$ tal que $\left\{a_{k}: k \in I\right\} \in p$ e $\left\{\left[k, a_{k}\right]: k \in I\right\}$ são intervalos 2 a 2 disjuntos de $\omega$. 
Seja $\left\{i_{k}: k \in \omega\right\}$ enumeração crescente de $I$. Temos que $i_{k}<a_{i_{k}}<i_{k+1}, \forall k<\omega$. Usando isto, obtemos

$$
F_{i_{k}} \cup \bigcup_{\xi \in F_{i_{k}}} f_{\xi}\left(a_{i_{k}}\right) \subseteq F_{i_{k+1}} \cup \bigcup_{\xi \in F_{i_{k}}} \bigcup_{m<i_{k+1}} f_{\xi}(m) \subseteq F_{i_{k+1}}, \forall k<\omega .
$$

Note que para cada $k<\omega,\left\{f_{\xi}\left(a_{i_{k}}\right): \xi \in F_{i_{k}}\right\} \cup\left\{\{\mu\}: \mu \in F_{i_{k}}\right\}$ é linearmente independente, uma vez que $a_{i_{k}} \in A_{i_{k}}$. Então, para $k \in[1, \omega)$ definimos $E_{k}=F_{i_{k}}$ e, para cada $k<\omega$, fazemos $b_{k}=a_{i_{k}}$. É evidente agora que os itens 2) e 3) são satisfeitos. Lembramos que $E_{0} \subseteq F_{i_{0}}, \omega \subseteq \bigcup_{n \in \omega} F_{n} \subseteq \bigcup_{k \in \omega} F_{i_{k}} \subseteq \bigcup_{k \in \omega} E_{k}$ e $B=\left\{b_{k}: k \in \omega\right\} \in p$.

Lema 3.2.4: Seja $p \in \omega^{*}$ um ultrafiltro seletivo. Para cada $\alpha<\mathfrak{c}$, existe um homomorfismo não-trivial $\phi_{\alpha}:[\mathfrak{c}]^{<\omega} \longmapsto\{0,1\}$ tal que

i) $\phi_{\alpha}(\{\xi\})=p-\lim \left\{\phi_{\alpha}\left(f_{\xi}(n)\right): n \in \omega\right\}, \forall \xi<\mathfrak{c}$;

ii) $\phi_{\alpha}\left(F_{\alpha}\right)=1$.

Demonstração: Tome $\alpha<c$. Aplicando o lema anterior a $E_{0}=F_{\alpha}$, obtemos uma sequência $\left\{b_{i}: i \in \omega\right\} \in p$ e uma sequência $\left\{E_{i}: 0<i<\omega\right\} \subseteq[c]^{<\omega}$ tais que valem os itens 1), 2) e 3) do lema anterior. Agora, suponha que para cada $i<\omega$, nós já tenhamos definido $\phi_{\alpha}$ em $\left[E_{i}\right]^{<\omega}$ de tal forma que $\phi_{\alpha}\left(F_{\alpha}\right)=1$ e $\phi_{\alpha}\left(f_{\xi}\left(b_{i}\right)\right)=\phi_{\alpha}(\{\xi\})$, $\forall \xi \in E_{i}$. Como $\left\{f_{\xi}\left(b_{i+1}\right): \xi \in E_{i+1}\right\} \cup\left\{\{\mu\}: \mu \in E_{i+1}\right\}$ é linearmente independente e $E_{i} \cup \bigcup_{\xi \in E_{i}} f_{\xi}\left(b_{i}\right) \subseteq E_{i+1}$, podemos estender $\phi_{\alpha}:\left[E_{i}\right]^{<\omega} \longmapsto\{0,1\}$ a um homomorfismo de $\left[E_{i+1}\right]^{<\omega}$ a $\{0,1\}$ de tal forma que $\phi_{\alpha}\left(f_{\xi}\left(b_{i+1}\right)\right)=\phi_{\alpha}(\{\xi\}), \forall \xi \in E_{i+1}$. Assim, definimos $\phi_{\alpha}$ em $[E]^{<\omega}$. Observe que $\phi_{\alpha}\left(f_{\xi}\left(b_{i}\right)\right)=\phi_{\alpha}(\{\xi\})$, para cada $\xi \in E_{i}$ e $i \in \omega$. Portanto, $\left\{n \in \omega: \phi_{\alpha}\left(f_{\xi}(n)\right)=\phi_{\alpha}(\{\xi\})\right\} \in p, \forall \xi \in E$. Nosso próximo objetivo é estender $\phi_{\alpha}$ a $[c]^{<\omega}$. Faremos isto por indução transfinita em $c \backslash E$. Seja $\gamma \in c \backslash E$ o menor ordinal para o qual $\phi_{\alpha}$ ainda não está definido. Temos que $\phi_{\alpha}$ está definido em $[E \cup \gamma]^{<\omega}$. Como $f_{\gamma}(n) \subseteq \gamma, \forall n \in \omega, \phi_{\alpha}(\{\mu\})$ já foi definido para todo $\mu<\gamma$ e $\{\{\gamma\}\} \cup\{\{\mu\}: \mu<\gamma\}$ é linearmente independente, temos que $\phi_{\alpha}$ pode ser estendido a $[E \cup(\gamma+1)]^{<\omega}$ de forma que 
$\phi_{\alpha}(\{\gamma\})=p-\lim \left\{\phi_{\alpha}\left(f_{\gamma}(n)\right): n \in \omega\right\}$. Segue-se o lema.

\subsection{Grupo enumeravelmente compacto cujo quadrado não é enumeravelmente compacto a partir de um grupo de van Douwen de ordem p.}

Mostraremos em ZFC que a existência de um grupo de van Douwen de ordem $p$ implica na existência de um grupo enumeravelmente compacto cujo quadrado não é enumeravelmente compacto. Na próxima secção obteremos o mesmo resultado a partir de um grupo de van Douwen livre. Finalmente, mostraremos que se existe um grupo abeliano enumeravelmente compacto sem sequências não-triviais convergentes, então existe um grupo do mesmo tipo que, em adição, é espaço vetorial sobre $\mathbb{Z}_{p}$ para algum primo $p$ ou é livre. Dessa forma, obtemos o resultado principal deste capítulo: a existência de um grupo de van Douwen implica em ZFC na existência de um grupo enumeravelmente compacto cujo quadrado não é enumeravelmente compacto. Todos os resultados desta secção podem ser encontrados em [11].

Lema 3.3.1: Seja $p$ um número primo e $G$ um grupo van Douwen de ordem $p$. Seja $\left\{f_{\xi}: \omega \leq \xi<\mathfrak{c}\right\}$ uma enumeração de todas as funções injetoras $f: \omega \longmapsto \mathbb{Z}_{p}^{(\mathfrak{c})}$ $\operatorname{com} \bigcup_{n \in \omega} \operatorname{supp}\left(f_{\xi}(n)\right) \subseteq \xi$ para cada $\xi \in[\omega, \mathfrak{c})$. Então, existem $\left\{x_{\xi}: \xi<\mathfrak{c}\right\} \subseteq G$ e $\left\{p_{\xi}: \omega \leq \xi<\mathfrak{c}\right\} \subseteq \omega^{*}$ tais que:

a) $\left\{x_{\xi}: \xi<\mathfrak{c}\right\}$ é l.i.; 
b) $x_{\xi}$ é $\quad$ o $p_{\xi}$-limite de $\left\{x_{f_{\xi}(n)}: n \in \omega\right\}, \quad \forall \xi \in[\omega, \mathfrak{c}) . \quad\left(x_{f_{\xi}(n)}=\right.$ $\left.\sum_{\lambda \in \operatorname{supp} f_{\xi}(n)} f_{\xi}(n)(\lambda) \cdot x_{\lambda}, \operatorname{supp}_{\xi}(n) \subseteq \xi, \forall n \in \xi\right)$.

Demonstração: Primeiramente, cuidemos da enumeração $\left\{f_{\xi}: \omega \leq \xi<\mathfrak{c}\right\}$. Sabemos que $\left|\left(\mathbb{Z}_{p}^{(\mathfrak{c})}\right)^{\omega}\right|=\mathfrak{c}$. Considere então $A=\left\{f \in\left(\mathbb{Z}_{p}^{(\mathfrak{c})}\right)^{\omega}: f\right.$ é injetora $\}$ e $\left\{g_{\xi}: \xi<\mathfrak{c}\right\}$ uma enumeração dos elementos de $A$ de tal forma que dada $f \in A,\left|\left\{\xi: g_{\xi}=f\right\}\right|=\mathfrak{c}$. Seja agora $h: \omega \longmapsto \mathbb{Z}_{p}{ }^{(\mathfrak{c})}$ tal que $h(n)=\mathcal{X}_{\{n\}}: \mathfrak{c} \longmapsto \mathbb{Z}_{p}$. Considere $\xi$ tal que $\omega \leq \xi<\mathfrak{c}$. Se $\bigcup_{n \in \omega} \operatorname{supp}\left(g_{\xi}(n)\right) \subseteq \xi$, defina $f_{\xi}=g_{\xi}$. Se $\bigcup_{n \in \omega} \operatorname{supp}\left(g_{\xi}(n)\right) \nsubseteq \xi$, então defina $f_{\xi}=h$ de forma que $\bigcup_{n \in \omega} \operatorname{supp}\left(f_{\xi}(n)\right) \subseteq \xi, \forall \xi<\mathfrak{c}$. Vamos garantir agora que $A \subseteq\left\{f_{\xi}: \omega \leq \xi<\mathfrak{c}\right\}$. Se $f \in A$, então $\exists \gamma<\mathfrak{c}$ tal que $\bigcup_{n \in \omega} \operatorname{supp}(f(n)) \subseteq \gamma$. Como $\left|\left\{\xi: g_{\xi}=f\right\}\right|=\mathfrak{c}$, existe $\xi>\gamma$ tal que $g_{\xi}=f, \operatorname{logo} \bigcup_{n \in \omega} \operatorname{supp}\left(g_{\xi}(n)\right) \subseteq \gamma \subseteq \xi$. Assim, $f_{\xi}=g_{\xi}=f$ está na enumeração.

O grupo $G$ é um espaço vetorial sobre $\mathbb{Z}_{p}$. Assim, existe um subconjunto linearmente independente $\left\{x_{n}: n \in \omega\right\}$ contido em $G$. As condições a) e b) são satisfeitas com $\omega$ no lugar de $\mathfrak{c}$ - condição b) de forma vazia. Suponha que $\left\{x_{\xi}: \xi<\mu\right\}$ e $\left\{p_{\xi}: \omega \leq \xi<\mu\right\}$ estão definidos para algum $\mu<\mathfrak{c}$ de tal forma que as hipóteses de indução a) e b) são satisfeitas com $\mu$ no lugar de $\mathfrak{c}$.

Pelo fato de que $\bigcup_{n \in \omega} \operatorname{supp}\left(f_{\mu}(n)\right) \subseteq \mu$, temos que a sequência $\left\{x_{f_{\mu}(n)}: n \in \omega\right\}$ já está definida e é injetora. Como $G$ é enumeravelmente compacto e não possui sequências nãotriviais convergentes, a sequência $\left\{x_{f_{\mu}(n)}: n \in \omega\right\}$ tem pelo menos $\mathfrak{c}$ pontos de acumulação (lema 1.1.9). O grupo gerado por $\left\{x_{\xi}: \xi<\mu\right\}$ tem cardinalidade menor que c. Assim, existe um ponto de acumulação $x_{\mu}$ de $\left\{x_{f_{\mu}(n)}: n \in \omega\right\}$ que não pertence ao subgrupo gerado por $\left\{x_{\xi}: \xi<\mu\right\}$. O conjunto $\left\{x_{\xi}: \xi \leq \mu\right\}$ será então linearmente independente e podemos obter $p_{\mu}$ ultrafiltro livre tal que $x_{\mu}$ é $p_{\mu}$-limite de $\left\{x_{f_{\mu}(n)}: n \in \omega\right\}$. Assim, finalizamos a construção de $\left\{x_{\xi}: \xi<\mathfrak{c}\right\} \subseteq G$ e $\left\{p_{\xi}: \omega \leq \xi<\mathfrak{c}\right\} \subseteq \omega^{*}$ satisfazendo os itens a) e b).

Lema 3.3.2: Sejam $G,\left\{f_{\xi}: \omega \leq \xi<\mathfrak{c}\right\},\left\{x_{\xi}: \xi<\mathfrak{c}\right\}$ e $\left\{p_{\xi}: \omega \leq \xi<\mathfrak{c}\right\}$ como no lema anterior. Então, existe uma família $\left\{z_{\xi}: \xi<\mathfrak{c}\right\} \subseteq G \times\left(\mathbb{Z}_{p}\right)^{\mathfrak{c}}$ tal que: 
(I) $\left\{z_{\xi}: \xi<\mathfrak{c}\right\}$ é conjunto l.i.;

(II) $z_{\xi}$ é o $p_{\xi^{-}}$-limite de $\left\{z_{f_{\xi}(n)}: n \in \omega\right\}, \forall \xi \in[\omega, \mathfrak{c})$;

(III) $\forall A \subseteq \omega, \exists \alpha \in \mathfrak{c}$ tal que $\left\{n \in \omega: z_{n}(\alpha)=1\right\}=A$ e $\left\{n \in \omega: z_{n}(\alpha)=0\right\}=\omega \backslash A$;

(IV) Se $p, q \in \omega^{*}$ e $p \neq q$, então para quaisquer $k_{0}, k_{1}, l_{0}, l_{1} \in \mathbb{Z}_{p}$ com $\left(k_{0}, k_{1}, l_{0}, l_{1}\right) \neq(0,0,0,0)$ teremos que $p-\lim \left\{\sum_{j<2} k_{j} z_{2 n+j}: n \in \omega\right\}$ é diferente de $q-\lim \left\{\sum_{j<2} l_{j} z_{2 n+j}: n \in \omega\right\}$.

Demonstração: Por hipótese, $\left\{x_{\xi}: \xi<\mathfrak{c}\right\} \subseteq G$ satisfaz:

a) $\left\{x_{\xi}: \xi<\mathfrak{c}\right\}$ é linearmente independente;

b) $x_{\xi}=p_{\xi}-\lim \left\{x_{f_{\xi}(n)}: n \in \omega\right\}, \forall \xi \in[\omega, \mathfrak{c})$.

Para cada $\alpha<\mathfrak{c}$, defina $G_{\alpha}$ como o grupo gerado por $\left\{x_{\xi}: \xi<\alpha\right\}$. Sabemos que $\left\{x_{n}: n \in \omega\right\}$ é linearmente independente. Daí,

c) para cada $A \subseteq \omega$ existe um homomorfismo $\phi_{A}\left\lceil G_{\omega}: G_{\omega} \longmapsto \mathbb{Z}_{p}\right.$ tal que $\phi_{A}\left(x_{n}\right)=1$, $\forall n \in A$, e $\phi_{A}\left(x_{n}\right)=0$, se $n \in \omega \backslash A$.

Estendemos $\phi_{A}$ de $G_{\omega}$ até $G_{\mathfrak{c}}$ de modo que satisfaça:

d) $\phi_{A}\left(x_{\xi}\right)=p_{\xi}-\lim \left\{\phi_{A}\left(x_{f_{\xi}(n)}\right): n \in \omega\right\}$.

Suponha que $\phi_{A} \uparrow G_{\alpha}$ já esteja definido e satisfaça a condição d) para todo $\alpha<\xi$. O homomorfismo $\phi_{A}$ é definido em $\bigcup_{\alpha<\xi} G_{\alpha}$. Seja $\xi$ ordinal limite. Se $x \in G_{\xi}$, então $x=\sum_{\lambda \in F} c_{\lambda} x_{\lambda}, F \in[\xi]^{<\omega}$. Seja $\psi$ ordinal tal que $F \subseteq \eta \subseteq \xi$. Como $\phi_{A} \uparrow G_{\eta}$ já está definido temos que $\phi_{A}(x)=\left(\phi_{A}\left\lceil G_{\eta}\right)(x)\right.$. Daí, obtemos que $\phi_{A}$ está definido em $G_{\xi}$. Provaremos agora que vale d). Sabemos que $\bigcup_{n \in \omega} \operatorname{supp}\left(f_{\xi}(n)\right) \subseteq \xi$, logo a sequência $\left\{\phi_{A}\left(x_{f_{\xi}(n)}\right): n \in \omega\right\}$ já está definida, já que $x_{f_{\xi}(n)} \in G_{\xi}, \forall n \in \omega$. Como $\mathbb{Z}_{p}$ é finito e discreto temos que é $p_{\xi^{-}}$ compacto e, portanto, podemos fazer $\phi_{A}\left(x_{\xi}\right)=p_{\xi}-\lim \left\{\phi_{A}\left(x_{f_{\xi}(n)}\right): n \in \omega\right\}$. Desta forma, 
$\phi_{A}$ está definida em $G_{\xi}$ e d) é satisfeito. Para o caso de $\xi=\beta+1$ ordinal sucessor, temos que $G_{\beta}$ já está definido e basta, então, repetir o raciocínio anterior para fazer valer o item d).

Defina $z_{\xi}=\left(x_{\xi},\left\{\phi_{A}\left(x_{\xi}\right): A \in P(\omega)\right\}\right) \in G \times\left(\mathbb{Z}_{p}\right)^{P(\omega)} \simeq G \times\left(\mathbb{Z}_{p}\right)^{\mathfrak{c}}($ associe cada $\alpha \in \mathfrak{c}$ a um único $A \in P(\omega)$ ). Provaremos agora que valem os itens (I), (II), (III) e (IV) do enunciado do lema.

(I) A família $\left\{z_{\xi}: \xi<\mathfrak{c}\right\}$ é l.i., pois suponha $\sum_{\lambda \in F} n_{\lambda} z_{\lambda}=0$, com $F \in[\mathfrak{c}]^{<\omega}$ e $n_{\lambda} \in \mathbb{Z}_{p}$, $\forall \lambda \in F$. Temos que $\sum_{\lambda \in F} n_{\lambda} z_{\lambda}=0 \Longrightarrow \sum_{\lambda \in F} n_{\lambda} x_{\lambda}=0 \Longrightarrow n_{\lambda} x_{\lambda}=0 \Longrightarrow n_{\lambda}=0, \forall \lambda \in F$, já que $\left\{x_{\xi}: \xi<\mathfrak{c}\right\}$ é linearmente independente e $G$ é grupo de ordem $p$. Logo, $n_{\lambda} z_{\lambda}=0$, $\forall \lambda \in F$ e $\left\{z_{\xi}: \xi<\mathfrak{c}\right\}$ é linearmente independente.

(II) Tomando $z_{\xi}$ qualquer, considere uma vizinhança aberta $V$ de $z_{\xi}$ tal que $V=U \times \prod_{\alpha \in F} V_{\alpha} \times \prod_{\alpha \in \mathfrak{c} \backslash F} \mathbb{Z}_{p}$, sendo $U$ vizinhança aberta de $x_{\xi}, V_{\alpha}$ de $\phi_{\alpha}\left(x_{\xi}\right)$ e $F \in[\mathfrak{c}]^{<\omega}$. Se chamarmos $A=\left\{n \in \omega: x_{f_{\xi}(n)} \in U\right\}$ e $A_{\alpha}=\left\{n \in \omega: \phi_{A}\left(x_{f_{\xi}(n)}\right) \in V_{\alpha}\right\}$, teremos da definição de $p_{\xi}$-limite que $A \in p_{\xi}$ e $A_{\alpha} \in p_{\xi}, \forall \alpha \in F$, pois $x_{\xi}=p_{\xi}-\lim \left\{x_{f_{\xi}(n)}: n \in \omega\right\}$ e vale d). Como $F$ é finito e $p_{\xi}$ é filtro temos que $A \cap \bigcap_{\alpha \in F} A_{\alpha} \in p_{\xi}$, ou seja, $\left\{n \in \omega: z_{f_{\xi}(n)} \in V\right\} \in p_{\xi}$ e vale o item (II). Como $\left\{f_{\xi}: \omega \leq \xi<\mathfrak{c}\right\}$ contém todas as $f \in\left(\mathbb{Z}_{p}{ }^{(\mathfrak{c})}\right)^{\omega}$ injetoras teremos que $\left\langle\left\{z_{\xi}: \xi<\mathfrak{c}\right\}\right\rangle$ será enumeravelmente compacto.

(III) Segue-se diretamente da definição de $z_{\xi}$ e do item c).

(IV) Se $p, q \in \omega^{*}$ são distintos, então existe, digamos, $A_{p} \in p \backslash q$ e existe algum $A_{q} \in q$ tal que $A_{p} \cap A_{q}=\emptyset$. Suponha, sem perda de generalidade, que $k_{0} \neq 0$. Considere $B_{0}=\left\{2 n: n \in A_{p}\right\}$. Pelo item c), $\phi_{B_{0}}\left(x_{2 n}\right)=1$ e $\phi_{B_{0}}\left(x_{2 n+1}\right)=0, \forall n \in A_{p}$, e $\phi_{B_{0}}\left(x_{2 n}\right)=$ $=\phi_{B_{0}}\left(x_{2 n+1}\right)=0, \forall n \in A_{q}$. Portanto, o p-limite da sequência

$$
\left\{\phi_{B_{0}}\left(k_{0} x_{2 n}+k_{1} x_{2 n+1}\right): n \in \omega\right\}
$$


é $k_{0}$ - uma vez que $\left\{n \in \omega: \phi_{B_{0}}\left(k_{0} x_{2 n}+k_{1} x_{2 n+1}\right)=k_{0}\right\} \supseteq A_{p}$; e o q-limite da sequência $\left\{\phi_{B_{0}}\left(l_{0} x_{2 n}+l_{1} x_{2 n+1}\right): n \in \omega\right\}$ é 0. Logo, os limites em (IV) são distintos.

Lema 3.3.3: Seja $\mathcal{F}=\left\{f_{\xi}: \omega \leq \xi<\mathfrak{c}\right\}$ uma enumeração de todas as funções injetoras $f: \omega \longmapsto \mathbb{Z}_{p}{ }^{(\mathfrak{c})}$ tal que $\bigcup_{n \in \omega} \operatorname{supp}\left(f_{\xi}(n)\right) \subseteq \xi$ para cada $\xi \in[\omega, \mathfrak{c})$ e cada $f: \omega \longmapsto \mathbb{Z}_{p}(\mathfrak{c})$ injetora aparece $\mathfrak{c}$ vezes na enumeração. Sejam $\left\{z_{\xi}: \xi<\mathfrak{c}\right\}$ e $\left\{p_{\xi}: \omega \leq \xi<\mathfrak{c}\right\}$ como no lema anterior para $\mathcal{F}$ acima. Então, existem famílias $\left\{I_{\xi}: \xi<\mathfrak{c}\right\},\left\{\alpha_{\xi}: \xi<\mathfrak{c}\right\},\left\{S_{\xi}: \xi<\mathfrak{c}\right\}$ e $\left\{P_{\xi}: \xi<\mathfrak{c}\right\}$ que satisfazem:

i) $I_{\xi}=\omega \cup\left\{\alpha_{\eta}: \eta<\xi\right\}, \forall \xi<\mathfrak{c}, \operatorname{com} \alpha_{\eta} \in \mathfrak{c}, \forall \eta<\mathfrak{c}$;

ii) $S_{\xi} \subseteq \mathfrak{c}, \forall \xi<\mathfrak{c}$;

iii) $\left(I_{\xi} \cup\left\{\alpha_{\xi}\right\}\right) \cap S_{\xi}=\emptyset, \forall \xi<\mathfrak{c}$;

iv) $f_{\alpha_{\xi}}=f_{\beta_{\xi}}$, sendo $\beta_{\xi}$ o menor ordinal $\beta$ em $[\omega, \mathfrak{c})$ para o qual $\bigcup_{n \in \omega} \operatorname{supp}\left(f_{\beta}(n)\right) \subseteq I_{\xi}$ e $f_{\beta} \neq f_{\alpha_{\eta}}, \forall \eta<\xi$

(v) para cada $p \in \omega^{*}$, temos que $p \in P_{\xi}$ se, e somente se, $p-\lim \left\{z_{2 n+j}: n \in \omega\right\} \in$ $\in\left\langle\left\{z_{\eta}: \eta<\mathfrak{c}\right\}\right\rangle, \forall j<2$, e $\exists k_{0}, k_{1} \in \mathbb{Z}_{p} \operatorname{com}\left(k_{0}, k_{1}\right) \neq(0,0)$ tal que $p-\lim \left\{k_{0} z_{2 n}+k_{1} z_{2 n+1}: n \in \omega\right\} \in\left\langle\left\{z_{\eta}: \eta \in I_{\xi}\right\}\right\rangle$

(vi) se $p \in P_{\xi}$, então existe $j<2$ tal que $p-\lim \left\{z_{2 n+j}: n \in \omega\right\} \notin\left\langle\left\{z_{\eta}: \eta \in \mathfrak{c} \backslash S_{\xi}\right\}\right\rangle$;

(vii) $\left|P_{\xi}\right| \leq|\xi|+\omega, \forall \xi<\mathfrak{c}$

(viii) $\left|S_{\xi}\right| \leq|\xi|+\omega, \forall \xi<\mathfrak{c}$;

(ix) se $\gamma<\xi<\mathfrak{c}$, então $P_{\gamma} \subseteq P_{\xi}$ e se $\xi$ é limite, então $P_{\xi}=\bigcup_{\gamma<\xi} P_{\gamma}$;

(x) se $\gamma<\xi<\mathfrak{c}$, então $S_{\gamma} \subseteq S_{\xi}$ e se $\xi$ é limite, então $S_{\xi}=\bigcup_{\gamma<\xi}^{\gamma} S_{\gamma}$.

Demonstração: Durante a construção por indução, denote $H_{\xi}$ o grupo gerado por $\left\{z_{\eta}: \eta \in I_{\xi}\right\}$. Note que $H_{\xi}=\left\{z_{h}: h \in \mathbb{Z}_{p}{ }^{\left(I_{\xi}\right)}\right\}$.

Estágio 0. Defina $I_{0}=\omega$. Então i) é satisfeito. Vamos provar que com esta definição teremos $P_{0}=\emptyset$. Para isso, provaremos que a sequência $\left\{k_{0} z_{2 n}+k_{1} z_{2 n+1}: n \in \omega\right\}$ não possui 
ponto de acumulação em $H_{0}=\left\{z_{\eta}: \eta \in I_{0}\right\}$. Sejam $\left(k_{0}, k_{1}\right) \in \mathbb{Z}_{p} \times \mathbb{Z}_{p} \operatorname{com}\left(k_{0}, k_{1}\right) \neq(0,0)$ e assuma, sem perda de generalidade, $k_{0} \neq 0$. Considere $z_{h} \in H_{0}\left(h \in \mathbb{Z}_{p}{ }^{(\omega)}\right)$ e $k \in \omega$ tais que $\operatorname{supp}(h) \subseteq 2 k$. Pelo item (III) do lema anterior, existe $\alpha<\mathfrak{c}$ tal que $\left\{n \in \omega: z_{n}(\alpha)=1\right\}$ é igual a $\{2 n: n \in \omega\} \backslash 2 k$ e $\left\{n \in \omega: z_{n}(\alpha)=0\right\}=\{2 n+1: n \in \omega\} \cup 2 k$. Dessa forma, $z_{h}(\alpha)=0, z_{2 n}(\alpha)=1$ e $z_{2 n+1}(\alpha)=0, \forall n \geq k$. Assim, $k_{0} z_{2 n}(\alpha)+k_{1} z_{2 n+1}(\alpha)=k_{0} \neq 0$, $\forall n \geq k$. Portanto, $z_{h}$ não é ponto de acumulação de $\left\{k_{0} z_{2 n}+k_{1} z_{2 n+1}: n \in \omega\right\}$ e como foi tomado qualquer em $H_{0}$ temos que a sequência $\left\{k_{0} z_{2 n}+k_{1} z_{2 n+1}: n \in \omega\right\}$ não possui ponto de acumulação em $H_{0}$. Logo, $P_{0}=\emptyset$ e, portanto, os itens (vi), (vii) e (ix) são trivialmente satisfeitos. Defina agora $S_{0}=\emptyset$ e faça $\alpha_{0}=\omega$. Os itens (ii), (iii), (iv), (viii) e (x) são claramente satisfeitos.

Caso $\xi=\gamma+1$. Por hipótese, $\alpha_{\eta}$ já está definido para todo $\eta \leq \gamma$. Portanto, $I_{\xi}=\omega \cup\left\{\alpha_{\eta}: \eta<\xi\right\}$ satisfaz o item i). Seja $P_{\xi}$ definido de acordo com o item v). Para cada $p \in P_{\xi}$, existem $k_{p}, l_{p} \in \mathbb{Z}_{p}$, com pelo menos um deles diferente de zero, tais que $a_{p}=p-\lim \left\{k_{p} z_{2 n}+l_{p} z_{2 n+1}: n \in \omega\right\} \in H_{\xi}$. Segue-se do item (IV) do lema anterior que $\left\{a_{p}: p \in P_{\xi}\right\}$ são dois a dois distintos. Logo, $\left|P_{\xi}\right| \leq\left|H_{\xi}\right|=|\xi|+\omega$ e o item vii) é satisfeito. O item ix) para o caso sucessor sai da definição de $P_{\xi}$ em v).

Definiremos agora $S_{\xi}$. Seja $q \in P_{\xi} \backslash P_{\gamma}$. Segue-se de v) que $q-\lim \left\{z_{2 n+j}: n \in \omega\right\}$ $\in\left\langle\left\{z_{v}: v<\mathfrak{c}\right\}\right\rangle, \forall j<2$, e para todo $\left(m_{0}, m_{1}\right) \in \mathbb{Z}_{p} \times \mathbb{Z}_{p} \operatorname{com}\left(m_{0}, m_{1}\right) \neq(0,0)$ teremos $q-\lim \left\{m_{0} z_{2 n}+m_{1} z_{2 n+1}: n \in \omega\right\} \notin H_{\gamma}$ - caso contrário, teríamos $q \in P_{\gamma}$. Em particular,

(*) $q-\lim \left\{z_{2 n+j}: n \in \omega\right\} \notin H_{\gamma}, \forall j<2$.

Afirmamos que existe $j<2$ tal que $q-\lim \left\{z_{2 n+j}: n \in \omega\right\} \notin H_{\xi}$. De fato, suponha por contradição que a afirmação seja falsa. Por $(*), z(j)=q-\lim \left\{z_{2 n+j}: n \in \omega\right\} \in H_{\xi} \backslash H_{\gamma}$, $\forall j<2$. Então, existe $h_{j} \in \mathbb{Z}_{p}{ }^{(\mathfrak{c})} \operatorname{com} \alpha_{\gamma} \in \operatorname{supp}\left(h_{j}\right) \subseteq I_{\gamma} \cup\left\{\alpha_{\gamma}\right\}$ tal que $z(j)=z_{h_{j}}$. Seja $s_{j}=h_{j}\left(\alpha_{\gamma}\right)$. Então, $s_{j} \neq 0$ e $\operatorname{supp}\left(s_{1} h_{0}-s_{0} h_{1}\right) \subseteq I_{\gamma}$. Daí,

$$
q-\lim \left\{s_{1} z_{2 n}+\left(-s_{0}\right) z_{2 n+1}: n \in \omega\right\}=s_{1} z(0)+\left(-s_{0}\right) z(1)=z_{s_{1} h_{0}+\left(-s_{0}\right) h_{1}} \in H_{\gamma} .
$$


Logo, pela condição v) temos que $q \in P_{\gamma}$, o que é contradição.

A afirmação do parágrafo anterior diz que para cada $q \in P_{\xi} \backslash P_{\gamma}$ existem $j_{q}<2, h_{q} \in \mathbb{Z}_{p}(\mathfrak{c})$ $\operatorname{com} \operatorname{supp}\left(h_{q}\right) \backslash I_{\xi} \neq \emptyset$ tal que o $q-\lim \left\{z_{2 n+j_{q}}: n \in \omega\right\}$ é igual a $z_{h_{q}}$. Fixe $\mu_{q} \in \operatorname{supp}\left(h_{q}\right) \backslash I_{\xi}$, $\forall q \in P_{\xi} \backslash P_{\gamma}$, e faça $S_{\xi}=S_{\gamma} \cup\left\{\mu_{q}: q \in P_{\xi} \backslash P_{\gamma}\right\}$. Os itens ii), vi), viii) e x) são satisfeitos.

Seja $\beta_{\xi}$ como no item iv). Pela escolha de $\left\{f_{\xi}: \omega \leq \xi<\mathfrak{c}\right\}$, existe uma quantidade não-enumerável de $f_{\mu}{ }^{\prime} s$ que são iguais a $f_{\beta_{\xi}}$. Podemos tomar $\alpha_{\xi}$ tal que $f_{\alpha_{\xi}}=f_{\beta_{\xi}}$ e $\alpha_{\xi} \in \mathfrak{c} \backslash\left(I_{\xi} \cup S_{\xi}\right)$. Valem os itens iii) e iv).

Daí, os itens i) - x) são satisfeitos para o caso sucessor.

Caso $\xi$ ordinal limite. Defina $P_{\xi}=\bigcup_{\eta<\xi} P_{\eta}$ como em ix), $S_{\xi}=\bigcup_{\eta<\xi} S_{\eta}$ como em x) e $I_{\xi}=\bigcup_{\eta<\xi} I_{\eta}=\omega \cup\left\{\alpha_{\eta}: \eta<\xi\right\}$ como em i). Os itens ii), vii) e viii) são claramente satisfeitos.

Veremos se a definição de $P_{\xi}$ dada no item v) é equivalente a $P_{\xi}=\bigcup_{\eta<\xi} P_{\eta}$. Seja $p \in P_{\xi}$. Existe $\mu<\xi$ tal que $p \in P_{\mu}$. Pelo item v) para $\mu$, existem $k_{0}, k_{1} \in \mathbb{Z}_{p}$ $\operatorname{com}\left(k_{0}, k_{1}\right) \neq(0,0)$ tais que $p-\lim \left\{k_{0} z_{2 n}+k_{1} z_{2 n+1}: n \in \omega\right\} \in\left\langle\left\{z_{\eta}: \eta<I_{\mu}\right\}\right\rangle \subseteq$ $\subseteq\left\langle\left\{z_{\eta}: \eta<I_{\xi}\right\}\right\rangle$. Pelo outro lado, suponha que existam $k_{0}, k_{1} \in \mathbb{Z}_{p} \operatorname{com}\left(k_{0}, k_{1}\right) \neq(0,0)$ tais que

$p-\lim \left\{k_{0} z_{2 n}+k_{1} z_{2 n+1}: n \in \omega\right\} \in\left\langle\left\{z_{\eta}: \eta<I_{\mu}\right\}\right\rangle \subseteq\left\langle\left\{z_{\eta}: \eta<I_{\xi}\right\}\right\rangle$.

Então, existe $\lambda<\xi$ tal que o p-limite de $\left\{k_{0} z_{2 n}+k_{1} z_{2 n+1}: n \in \omega\right\}$ está em $\left\langle\left\{z_{\eta}: \eta<I_{\lambda}\right\}\right\rangle$. Pelo item v) para $\lambda$, segue-se que $p \in P_{\lambda} \subseteq P_{\xi}$. Logo, as duas definições são equivalentes.

Vale o item vi). De fato, se $p \in P_{\xi}$, então existe $\beta<\xi$ tal que $p \in P_{\beta}$. Pelo item vi) para $\beta$, existe $j<2$ tal que $p-\lim \left\{z_{2 n+j}: n \in \omega\right\} \notin\left\langle\left\{z_{\eta}: \eta<\mathfrak{c} \backslash S_{\beta}\right\}\right\rangle$. Pelo item x), $S_{\beta} \subseteq S_{\xi}$. Portanto, $p-\lim \left\{z_{2 n+j}: n \in \omega\right\} \notin\left\langle\left\{z_{\eta}: \eta<\mathfrak{c} \backslash S_{\xi}\right\}\right\rangle$ e vale o item vi) para $\xi$.

Seja $\beta_{\xi}$ como no item iv). Pela escolha de $\left\{f_{\xi}: \omega \leq \xi<\mathfrak{c}\right\}$, existe uma quantidade não-enumerável de $f_{\mu}{ }^{\prime} s$ que são iguais a $f_{\beta_{\xi}}$. Podemos tomar $\alpha_{\xi}$ tal que $f_{\alpha_{\xi}}=f_{\beta_{\xi}}$ e $\alpha_{\xi} \in \mathfrak{c} \backslash\left(I_{\xi} \cup S_{\xi}\right)$. Valem os itens iii) e iv).

Isto finaliza a nossa construção por indução. 
Lema 3.3.4: Seja p um número primo. Suponha que exista um grupo de van Douwen $G$ de ordem $p$. Então, existe um grupo enumeravelmente compacto cujo quadrado não o é.

Demonstração: Sejam $\left\{f_{\xi}: \omega \leq \xi<\mathfrak{c}\right\},\left\{z_{\xi}: \xi<\mathfrak{c}\right\},\left\{p_{\xi}: \omega \leq \xi<\mathfrak{c}\right\},\left\{I_{\xi}: \xi<\mathfrak{c}\right\}$, $\left\{\alpha_{\xi}: \xi<\mathfrak{c}\right\},\left\{S_{\xi}: \xi<\mathfrak{c}\right\}$ e $\left\{P_{\xi}: \xi<\mathfrak{c}\right\}$ como no lema anterior, de forma que i) - x) são satisfeitos. Seja $I=\bigcup_{\xi<\mathfrak{c}} I_{\xi}$ e seja $H=\left\langle\left\{z_{\xi}: \xi \in I\right\}\right\rangle=\left\{z_{h}: h \in \mathbb{Z}_{p}{ }^{(I)}\right\}$.

Temos que $H$ é enumeravelmente compacto. De fato, seja $\left\{t_{n}: n \in \omega\right\}$ sequência 1-1 em $H$. Então, existe $h_{n} \in \mathbb{Z}_{p}^{(I)}$ tal que $t_{n}=z_{h_{n}}, \forall n \in \omega$. Seja $\mu<\mathfrak{c}$ tal que $\bigcup_{n \in \omega} \operatorname{supp}\left(h_{n}\right) \subseteq I_{\mu}$ e seja $\xi<\mathfrak{c}$ o menor ordinal tal que $f_{\xi}(n)=h_{n}, \forall n \in \omega$. Pelo item iv), existe $\lambda<\mathfrak{c}$ tal que $\beta_{\lambda}=\xi$. Pelo item vi), $f_{\alpha_{\lambda}}=f_{\xi}$. Então, a sequência pode ser vista como $\left\{z_{f_{\alpha_{\lambda}}(n)}: n \in \omega\right\}$ e $z_{\alpha_{\lambda}} \in H$ será ponto de acumulação desta sequência, pelo item (II) do lema 3.3.2.

Provaremos agora que $H^{2}$ não é enumeravelmente compacto. Considere a sequência $\left\{\left(z_{2 n}, z_{2 n+1}\right): n \in \omega\right\}$. Mostraremos que esta sequência não tem p-limite para nenhum ultrafiltro $p \in \omega^{*}$.

Caso A. $p \in \omega^{*} \backslash P_{\mathfrak{c}}$.

Pelo item v), existe $j<2$ tal que $\left\{z_{2 n+j}: n \in \omega\right\}$ não possui p-limite no grupo gerado por $\left\{z_{\beta}: \beta<\mathfrak{c}\right\}$ ou para cada $j<2$ a sequência $\left\{z_{2 n+j}: n \in \omega\right\}$ não possui p-limite em $H_{\alpha}$, $\forall \alpha<\mathfrak{c}$. Em ambos os casos, existe $j<2$ tal que a sequência $\left\{z_{2 n+j}: n \in \omega\right\}$ não possui p-limite em $H$. Logo, $\left\{\left(z_{2 n}, z_{2 n+1}\right): n \in \omega\right\}$ não possui p-limite em $H \times H$.

Caso B. $p \in P_{\mathfrak{c}}$.

Pela definição de $P_{\mathfrak{c}}$, existe $\beta<\mathfrak{c}$ tal que $p \in P_{\beta}$. Existe $j<2$ tal que $\left\{z_{2 n+j}: n \in \omega\right\}$ não tem p-limite em $H$ pelo item vi), uma vez que $H=\left\langle\left\{z_{\eta}: \eta \in I\right\}\right\rangle$ e $I \cap S_{\beta+1}=\emptyset$. Portanto, a sequência $\left\{\left(z_{2 n}, z_{2 n+1}\right): n \in \omega\right\}$ não possui p-limite em $H \times H$. 


\subsection{Grupo enumeravelmente compacto cujo quadrado não é enumeravelmente compacto a partir de um grupo de van Douwen livre.}

Obteremos nesta secção resultados análogos aos da secção anterior para grupos abelianos livres. Primeiramente, vamos ao lema que obtém um resultado análogo ao lema 3.3.1 da secção anterior, mas a partir de um grupo livre de van Douwen $G$.

Lema 3.4.1: Seja $G$ um grupo livre de van Douwen. Seja $\left\{f_{\xi}: \omega \leq \xi<\mathfrak{c}\right\}$ uma enumeração de todas as funções injetoras $f: \omega \longmapsto \mathbb{Z}^{(\mathfrak{c})} \operatorname{com} \bigcup_{n \in \omega} \operatorname{supp}\left(f_{\xi}(n)\right) \subseteq \xi$ para cada $\xi \in[\omega, \mathfrak{c})$. Então, existem duas famílias $\left\{x_{\xi}: \xi<\mathfrak{c}\right\} \subseteq G$ e $\left\{p_{\xi}: \xi<\mathfrak{c}\right\} \subseteq \omega^{*}$ tais que

a) $\left\{x_{\xi}: \xi<\mathfrak{c}\right\}$ é um conjunto independente;

b) $x_{\xi}$ é o $p_{\xi^{-}}$-limite de $\left\{x_{f_{\xi}(n)}: n \in \omega\right\}, \forall \xi \in[\omega, \mathfrak{c})$.

Demonstração: Existe um conjunto independente $Y=\left\{y_{\alpha}: \alpha<\theta\right\}$ para algum cardinal $\theta$ maior ou igual a $\mathfrak{c}$ tal que $Y$ gera $G$, uma vez que a cardinalidade do grupo de van Douwen é maior ou igual a c. Assim, podemos tomar um conjunto independente $\left\{x_{n}: n \in \omega\right\}$ contido em $G$. As condições a) e b) são claramente satisfeitas para $\omega$ no lugar de $\mathfrak{c}$.

Suponha que $\left\{x_{\xi}: \xi<\mu\right\}$ foi obtido para algum $\mu<\mathfrak{c}$, satisfazendo a) e b) com $\mu$ no lugar de $\mathfrak{c}$. Do fato de que $\bigcup_{n \in \omega} \operatorname{supp}\left(f_{\mu}(n)\right) \subseteq \mu$, temos que a sequência $\left\{x_{f_{\mu}(n)}: n \in \omega\right\}$ já está definida e é 1-1. Temos também que o grupo $G$ é enumeravelmente compacto e não possui sequências não-triviais convergentes. Assim, a sequência $\left\{x_{f_{\mu}(n)}: n \in \omega\right\}$ tem pelo menos c pontos de acumulação.

Para cada $\xi<\mu$, seja $F_{\xi}$ um subconjunto finito de $\theta$ tal que $x_{\xi}$ é gerado por $\left\{y_{\eta}: \eta \in F_{\xi}\right\}$. 
Faça $F=\bigcup_{\xi<\mu} F_{\xi}$. Como o grupo gerado por $\left\{y_{\eta}: \eta \in F\right\}$ tem tamanho menor do que $\mathfrak{c}$, existe um ponto de acumulação $x_{\mu}$ de $\left\{x_{f_{\mu}(n)}: n \in \omega\right\}$ que não está no grupo $\left\langle\left\{y_{\eta}: \eta \in F\right\}\right\rangle$. Seja $p_{\mu}$ um ultrafiltro livre tal que $x_{\mu}$ é $p_{\mu}$-limite de $\left\{x_{f_{\mu}(n)}: n \in \omega\right\}$. O item b) é claramente satisfeito.

Basta provar agora que vale o item a) para $\mu$, ou seja, $\left\{x_{\xi}: \xi \leq \mu\right\}$ é independente. Sejam $n_{0}, \ldots, n_{k}$ inteiros diferentes de zero e $\xi_{0}, \ldots, \xi_{k}$ ordinais dois a dois distintos no máximo iguais a $\mu$. Por contradição, assuma que $n_{0} x_{\xi_{0}}+\ldots+n_{k} x_{\xi_{k}}=0$. Sem perda de generalidade, assuma que $\xi_{0}=\mu$. Então,

$(*) n_{0} x_{\xi_{0}}=-n_{1} x_{\xi_{1}}-\ldots-n_{k} x_{\xi_{k}}$ é elemento de $\left\langle\left\{y_{\eta}: \eta \in F\right\}\right\rangle$.

Como $x_{\xi_{0}} \notin\left\langle\left\{y_{\eta}: \eta \in F\right\}\right\rangle$, existe um elemento diferente de zero $z_{0} \in\left\langle\left\{y_{\eta}: \eta \in \theta \backslash F\right\}\right\rangle$ tal que

$(* *) x_{\xi_{0}}-z_{0} \in\left\langle\left\{y_{\eta}: \eta \in F\right\}\right\rangle$.

Por $(*)$ e $(* *)$ temos que $n_{0} z_{0}=n\left(z_{0}-x_{\xi_{0}}\right)+n x_{\xi_{0}} \in\left\langle\left\{y_{\eta}: \eta \in F\right\}\right\rangle$.

Portanto, $n_{0} z_{0} \in\left\langle\left\{y_{\eta}: \eta \in F\right\}\right\rangle \cap\left\langle\left\{y_{\eta}: \eta \in \theta \backslash F\right\}\right\rangle$. Como $\left\{y_{\eta}: \eta \in \theta\right\}$ é independente, segue-se que $n_{0} z_{0}=0$. Mas o grupo $G$ não possui elementos de ordem finita. Logo, $z_{0}=0$. Contradição.

Uma vez construído $\left\{x_{\xi}: \xi<\mathfrak{c}\right\}$ satisfazendo os itens a) e b) do lema anterior temos que para construir um grupo enumeravelmente compacto cujo quadrado não é enumeravelmente compacto para o caso de $G$ grupo livre requer apenas pequenas modificações nas demonstrações dos lemas análogos da secção anterior, trocando apenas " $\mathbb{Z}_{p}$ " por " $\mathbb{Z} "$ e "linearmente independente"por "independente". Não demonstraremos os lemas novamente, apenas daremos os seus enunciados com as modificações necessárias.

Lema 3.4.2: Sejam $G,\left\{f_{\xi}: \omega \leq \xi<\mathfrak{c}\right\},\left\{x_{\xi}: \xi<\mathfrak{c}\right\}$ e $\left\{p_{\xi}: \omega \leq \xi<\mathfrak{c}\right\}$ como no lema anterior. Então existe uma família $\left\{z_{\xi}: \xi<\mathfrak{c}\right\} \subseteq G \times \mathbb{Z}^{\mathfrak{c}}$ tal que: 
(I) $\left\{z_{\xi}: \xi<\mathfrak{c}\right\}$ é conjunto independente;

(II) $z_{\xi}$ é o $p_{\xi}$-limite de $\left\{z_{f_{\xi}(n)}: n \in \omega\right\}, \forall \xi \in[\omega, \mathfrak{c})$;

(III) $\forall A \subseteq \omega, \exists \alpha \in \mathfrak{c}$ tal que $\left\{n \in \omega: z_{n}(\alpha)=1\right\}=A$ e $\left\{n \in \omega: z_{n}(\alpha)=0\right\}=\omega \backslash A$;

(IV) Se $p, q \in \omega^{*}$ e $p \neq q$, então para quaisquer $k_{0}, k_{1}, l_{0}, l_{1} \in \mathbb{Z}_{p} \operatorname{com}\left(k_{0}, k_{1}, l_{0}, l_{1}\right)$ diferente de $(0,0,0,0)$ teremos $p-\lim \left\{\sum_{j<2} k_{j} z_{2 n+j}: n \in \omega\right\} \neq q-\lim \left\{\sum_{j<2} l_{j} z_{2 n+j}: n \in \omega\right\}$.

Lema 3.4.3: Seja $\mathcal{F}=\left\{f_{\xi}: \omega \leq \xi<\mathfrak{c}\right\}$ uma enumeração de todas as funções injetoras $f: \omega \longmapsto \mathbb{Z}^{(\mathfrak{c})}$ tal que $\bigcup_{n \in \omega} \operatorname{supp}\left(f_{\xi}(n)\right) \subseteq \xi$ para cada $\xi \in[\omega, \mathfrak{c})$ e cada $f: \omega \longmapsto \mathbb{Z}^{(\mathfrak{c})}$ injetora aparece $\mathfrak{c}$ vezes na enumeração. Sejam $\left\{z_{\xi}: \xi<\mathfrak{c}\right\}$ e $\left\{p_{\xi}: \omega \leq \xi<\mathfrak{c}\right\}$ que satisfazem os itens (I)-(IV) do lema anterior para $\mathcal{F}$ acima. Então existem famílias $\left\{I_{\xi}: \xi<\mathfrak{c}\right\},\left\{\alpha_{\xi}: \xi<\mathfrak{c}\right\}$, $\left\{S_{\xi}: \xi<\mathfrak{c}\right\}$ e $\left\{P_{\xi}: \xi<\mathfrak{c}\right\}$ que satisfazem:

i) $I_{\xi}=\omega \cup\left\{\alpha_{\eta}: \eta<\xi\right\}, \forall \xi<\mathfrak{c}, \operatorname{com} \alpha_{\eta} \in \mathfrak{c}, \forall \eta<\mathfrak{c}$;

ii) $S_{\xi} \subseteq \mathfrak{c}, \forall \xi<\mathfrak{c}$;

iii) $\left(I_{\xi} \cup\left\{\alpha_{\xi}\right\}\right) \cap S_{\xi}=\emptyset, \forall \xi<\mathfrak{c}$;

iv) $f_{\alpha_{\xi}}=f_{\beta_{\xi}}$, sendo $\beta_{\xi}$ o menor ordinal $\beta$ em $[\omega, \mathfrak{c})$ para o qual $\bigcup_{n \in \omega} \operatorname{supp}\left(f_{\beta}(n)\right) \subseteq I_{\xi}$ e $f_{\beta} \neq f_{\alpha_{\eta}}, \forall \eta<\xi$;

(v) para cada $p \in \omega^{*}$, temos que $p \in P_{\xi}$ se, e somente se, $p-\lim \left\{z_{2 n+j}: n \in \omega\right\} \in$ $\in\left\langle\left\{z_{\eta}: \eta<\mathfrak{c}\right\}\right\rangle, \forall j<2, \mathrm{e} \exists k_{0}, k_{1} \in \mathbb{Z} \operatorname{com}\left(k_{0}, k_{1}\right) \neq(0,0)$ tais que $p-\lim \left\{k_{0} z_{2 n}+k_{1} z_{2 n+1}\right.$ : $n \in \omega\} \in\left\langle\left\{z_{\eta}: \eta<I_{\xi}\right\}\right\rangle$.

(vi) se $p \in P_{\xi}$, então existe $j<2$ tal que $p-\lim \left\{z_{2 n+j}: n \in \omega\right\} \notin\left\langle\left\{z_{\eta}: \eta<\mathfrak{c} \backslash S_{\xi}\right\}\right\rangle$;

(vii) $\left|P_{\xi}\right| \leq|\xi|+\omega, \forall \xi<\mathfrak{c}$;

(viii) $\left|S_{\xi}\right| \leq|\xi|+\omega, \forall \xi<\mathfrak{c}$;

(ix) se $\gamma<\xi<\mathfrak{c}$, então $P_{\gamma} \subseteq P_{\xi}$ e se $\xi$ é limite, então $P_{\xi}=\bigcup_{\gamma<\xi} P_{\gamma}$;

(x) se $\gamma<\xi<\mathfrak{c}$, então $S_{\gamma} \subseteq S_{\xi}$ e se $\xi$ é limite, então $S_{\xi}=\bigcup_{\gamma<\xi} S_{\gamma}$. 


\subsection{Grupo enumeravelmente compacto cujo quadrado não é enumeravelmente compacto a partir de um ultrafiltro seletivo.}

O lema seguinte mostra que se existe um grupo abeliano enumeravelmente compacto sem sequências não-triviais convergentes, então existe um grupo do mesmo tipo que, em adição, é espaço vetorial sobre $\mathbb{Z}_{p}$ para algum primo $p$ ou é livre. Com os resultados obtidos nas secções anteriores deste capítulo teremos que a existência de um ultrafiltro seletivo implica, em ZFC, na existência de um grupo enumeravelmente compacto, mas cujo quadrado não o é.

Lema 3.5.1: Seja $G$ um grupo de van Douwen. Então, $G$ contém um subgrupo de van Douwen de ordem $p$ para algum número primo $p$ ou contém um subgrupo abeliano de van Douwen livre.

Demonstração: Dado um inteiro positivo $n$, denote por $G[n]$ o conjunto $\{g \in G: n g=0\}$. Denote por $t(G)$ o subgrupo de torção de $G$. Se $|t(G)| \geq \mathfrak{c}$, então existe um número positivo $n$ tal que $|G[n]| \geq \mathfrak{c}$, já que $\mathfrak{c}$ tem cofinalidade não-enumerável e $t(G)=\bigcup_{n \in \omega} G[n]$. Seja $N$ o menor inteiro para o qual $|G[N]| \geq \mathfrak{c} . G[N]$ é subgrupo fechado, pois é imagem inversa por função contínua do fechado $\{0\}$ e, portanto, um subgrupo de van Douwen. Seja $P$ um divisor primo de $N$. Afirmamos que $N=P$. De fato, se $N$ não é primo, então existe $M<N$ tal que $N=M P$. Sabemos que todo subgrupo fechado infinito de um grupo de van Douwen deve ter cardinalidade $\geq \mathfrak{c}$. Daí, pela minimalidade de $N$, devemos ter $|G[M]|<\omega$. Considere o homomorfismo $\phi: G[N] \longmapsto G[P]$ tal que $\phi(g)=M g$. É claro que o kernel de $\phi$ é $G[M]$ e pelo primeiro teorema do isomorfismo teremos que o grupo quociente $G[N] / G[M]$ será isomorfo a um subgrupo de $G[P]$. Como 
$G[M]$ é subgrupo finito e $|G[N]| \geq \mathfrak{c}$ obtemos que $|G[N] / G[M]| \geq \mathfrak{c}$ fazendo com que $|G[P]| \geq \mathfrak{c}$, contrariando a minimalidade de $N$. Logo, $N=P$ é primo.

Agora, suponha que $|t(G)|<\mathfrak{c}$. Seja $\left\{f_{\xi}: 0<\xi<\mathfrak{c}\right\}$ uma enumeração de todas as funções injetoras $f: \omega \longmapsto \mathbb{Z}^{(\mathfrak{c})}$ tais que $\bigcup_{n \in \omega} \operatorname{supp}\left(f_{\xi}(n)\right) \subseteq \xi, \forall \xi<\mathfrak{c}$. Encontraremos uma família independente $\left\{x_{\xi}: \xi<\mathfrak{c}\right\} \subseteq G$ que gera um grupo abeliano livre. Comecemos com um $x_{0}$ arbitrário tal que $x_{0} \in G \backslash t(G)$. Suponha que $\left\{x_{\beta}: \beta<\xi\right\}$ já esteja definido e que

a) $X_{\xi}=\left\{x_{\beta}: \beta<\xi\right\}$ é uma base para um grupo livre;

b) $x_{\beta}$ é ponto de acumulação de $\left\{x_{f_{\beta}(n)}: n \in \omega\right\}, \forall \beta \in(0, \xi)$.

Definiremos $x_{\xi}$. Seja $\mathcal{A}$ o conjunto de todos os pontos de acumulação de $\left\{x_{f_{\xi}(n)}: n \in \omega\right\}$ distintos do elemento neutro do grupo. Suponhamos que exista $t \in \mathcal{A}$ tal que $\langle\{t\}\rangle \cap\left\langle X_{\xi} \cup t(G)\right\rangle=\{0\}$. Então, $X_{\xi} \cup\{t\}$ é base para um grupo abeliano livre. De fato, suponhamos que $n_{0}, \ldots, n_{l} \in \mathbb{Z}$ e $\mu_{1}, \ldots, \mu_{l}$ são ordinais dois a dois distintos $\leq \xi$ tais que $n_{0} t+n_{1} x_{\mu_{1}}+\ldots+n_{l} x_{\mu_{l}}=0$. Sem perda de generalidade, podemos considerar que $n_{0} \neq 0$. Então, $n_{0} t \in\langle\{t\}\rangle \cap\left\langle X_{\xi}\right\rangle$. Logo, temos que $n_{0} t=0 \Longrightarrow t \in\langle\{t\}\rangle \cap\left\langle X_{\xi} \cup t(G)\right\rangle \Longrightarrow$ $t=0$, o que é contradição. Portanto, para finalizar o lema basta provarmos que existe $t \in \mathcal{A}$ tal que $\langle\{t\}\rangle \cap\left\langle X_{\xi} \cup t(G)\right\rangle=\{0\}$.

Suponhamos por contradição que tal elemento $t \in \mathcal{A}$ não exista. Então, para cada $t \in \mathcal{A}$ existe um inteiro positivo $n_{t}$ tal que $n_{t} t \in H:=\left\langle X_{\xi} \cup t(G)\right\rangle$. Seja $\mathcal{A}_{n}=\left\{t \in \mathcal{A}: n_{t}=n\right\}$. Como $|\mathcal{A}| \geq \mathfrak{c}$ e $\mathfrak{c}$ tem cofinalidade não-enumerável, existe um inteiro positivo $n$ para o qual $\left|\mathcal{A}_{n}\right| \geq \mathfrak{c}$. Para cada $h \in H$, seja $\mathcal{A}_{n, h}=\left\{t \in \mathcal{A}_{n}: n t=h\right\}$. Como $|H|<\mathfrak{c}$, existe $h \in H$ tal que $\left|\mathcal{A}_{n, h}\right| \geq|t(G)|^{+}$. Tome $z \in \mathcal{A}_{n, h}$. Então, para cada $t \in \mathcal{A}_{n, h}$, $n(t-z)=n t-n z=h-h=0$. Assim, $t-z \in t(G)$ para todo $t \in \mathcal{A}_{n, h}$. Uma vez que $\left|\mathcal{A}_{n, h}\right| \geq|t(G)|^{+}$, temos que existem $t_{0}, t_{1} \in \mathcal{A}_{n, h}$ distintos tais que $t_{0}-z=t_{1}-z$. Portanto, $t_{0}=t_{1}$, o que é contradição.

Isto finaliza a contrução de $X=\left\{x_{\beta}: \beta<\mathfrak{c}\right\}$ satisfazendo os itens a) e b) com $\mathfrak{c}$ no 
lugar de $\xi$. O grupo gerado por $X$ é um subgrupo livre e enumeravelmente compacto de $G$ e, portanto, um grupo de van Douwen livre.

Lema 3.5.2 Suponha que exista um grupo abeliano livre de van Douwen G. Então, existe um grupo enumeravelmente compacto cujo quadrado não é enumeravelmente compacto. 


\section{Capítulo 4}

\section{Produto finito de grupos}

\section{enumeravelmente compactos.}

Neste capítulo obteremos um exemplo de grupo topológico enumeravelmente compacto $G$ tal que dado $k \in \omega$ qualquer $G^{k}$ é enumeravelmente compacto, mas $G^{k+1}$ não é enumeravelmente compacto. Este exemplo é obtido em [10], mas de forma diferente da aqui apresentada. Foi feito um esforço nesta dissertação no sentido de uniformizar a notação e as demonstrações relacionadas aos contra-exemplos obtidos neste capítulo e nos capítulos seguintes, daí o porquê deste exemplo ser diferente do obtido em [10]. Começamos com um lema muito parecido com o lema 3.1.3, que será utilizado nas construções deste capítulo e dos capítulos posteriores. Para o lema abaixo, considere $\kappa$ e $\alpha$ cardinais quaisquer.

Lema 4.1: Sejam $\left\{p_{j}: j<\omega\right\}$ uma família de ultrafiltros seletivos incomparáveis, $F$ um subconjunto finito de $\kappa \times \alpha, E$ um subconjunto enumerável de $\kappa \times \alpha$ contendo $F$ e $\left\{g_{\xi}: \xi \in E\right\}$ uma família enumerável de funções de $\omega$ em $[\kappa \times \alpha]^{<\omega}$. Se $\left\{S_{j}: j<\omega\right\}$ é uma família enumerável de subconjuntos de $\kappa \times \alpha$ tal que $\left\{\left[g_{\xi}\right]_{p_{j}}: \xi \in S_{j}\right\} \cup\left\{[\vec{\mu}]_{p_{j}}: \mu \in \kappa \times \alpha\right\}$ é linearmente independente em $\left([\kappa \times \alpha]^{<\omega}\right)^{\omega} / p_{j}$ para cada $j \in \omega$, então existem uma 
sequência crescente $\left\{b_{i}: i \in \omega\right\} \subseteq \omega$, uma função $r$ de $\omega$ em $\omega$ e uma sequência $\left\{E_{i}: i \in \omega\right\}$ de subconjuntos finitos de $E$ tais que:

(a) $F \subseteq E_{0}$

(b) $E=\bigcup_{i \in \omega} E_{i}$;

(c) $E_{i+1} \supseteq E_{i} \cup \bigcup\left\{g_{\xi}\left(b_{i}\right): \xi \in E_{i}\right\}$ para cada $i \in \omega$;

(d) $\left\{g_{\xi}\left(b_{i}\right): \xi \in E_{i} \cap S_{r(i)}\right\} \cup\left\{\{\mu\}: \mu \in E_{i}\right\}$ é linearmente independente para cada $i \in \omega$;

(e) $\left\{b_{k}: k \in r^{-1}(j)\right\} \in p_{j}$ para todo $j \in \omega$.

Além disso, se $\alpha \in \omega$ e $k \in \omega, E_{i}$ pode ser tomado para todo $i \in \omega$ de tal forma que:

(f) $\omega \times \alpha \subseteq E$ e $\omega \times \alpha \cap E_{i}=(k+1) N \times \alpha$, para algum $N \in \omega$.

Demonstração: Definiremos primeiramente uma família $\left\{F_{n}: n \in \omega\right\}$ de subconjuntos finitos de $E$. Defina:

(0) $F_{0}:=F$, e por indução em $\omega$, tome um subconjunto finito $F_{n+1}$ de $E$ tal que:

(1) $F_{n+1} \supseteq F_{n} \cup \bigcup\left\{g_{\beta}(m): m \leq n, \beta \in F_{n}\right\}$;

(2) $E=\bigcup_{n \in \omega} F_{n}$.

Seja $A_{n}^{j}=\left\{k \in \omega:\left\{g_{\xi}(k): \xi \in F_{n} \cap S_{j}\right\} \cup\left\{\{\mu\}: \mu \in F_{n}\right\}\right.$ é l.i. $\}, \forall j, n \in \omega$. Por hipótese, sabemos que $\left\{\left[g_{\xi}\right]_{p_{j}}: \xi \in S_{j}\right\} \cup\left\{[\vec{\mu}]_{p_{j}}: \mu \in E\right\}$ é linearmente independente. Como $F_{n}$ é finito, temos que $\triangle\left(\left\{\left[g_{\xi}\right]_{p_{j}}: \xi \in F_{n} \cap S_{j}\right\} \cup\left\{[\vec{\mu}]_{p_{j}}: \mu \in F_{n}\right\}\right) \neq[0]_{p_{j}}$, ou seja, existe $C_{j} \in p_{j}$ tal que $k \in C_{j} \Longrightarrow \triangle\left(\left\{g_{\xi}(k): \xi \in F_{n} \cap S_{j}\right\} \cup\left\{\mu: \mu \in F_{n}\right\}\right) \neq \emptyset$. Daí, $\left\{g_{\xi}(k): \xi \in F_{n} \cap S_{j}\right\} \cup\left\{\mu: \mu \in F_{n}\right\}$ é linearmente independente. Logo, $C_{j} \subseteq A_{n}^{j}$ e, portanto, $A_{n}^{j} \in p_{j}, \forall j, n \in \omega$.

Ultrafiltros seletivos são P-pontos, logo existe $A_{j}$ tal que:

(3) $A_{j} \in p_{j}$ e $A_{j} \backslash A_{n}^{j}$ é finito, $\forall j \in \omega$ e $\forall n \in \omega$.

Seja $h_{j}: \omega \longmapsto \omega$ função crescente tal que

(4) $A_{j} \backslash A_{n}^{j} \subseteq h_{j}(n), \forall j \in \omega$. 
Para todo $j \in \omega, h_{j}$ define uma partição em $\omega$ :

$\left[0, h_{j}(1)\right],\left\{\left[h_{j}(n)+1, h_{j}(n+1)\right]: n \geq 1\right\}$. Pela seletividade de $p_{j}$, existe $C_{j} \in p_{j}$ tal que $\left|\left[0, h_{j}(1)\right] \cap C_{j}\right|=1$ e $\left|\left[h_{j}(n)+1, h_{j}(n+1)\right] \cap C_{j}\right|=1, \forall n \geq 1$. Tome $B_{j}=\left(C_{j} \cap A_{j}\right) \backslash\left(\left[0, h_{j}(1)\right] \cap C_{j}\right)$. Então,

(5) $B_{j} \cap h_{j}(1)=\emptyset, B_{j} \subseteq A_{j}, B_{j} \in p_{j}$ e $\left|\left[h_{j}(n)+1, h_{j}(n+1)\right] \cap B_{j}\right| \leq 1, \forall j \in \omega$ e $\forall n \in \omega$.

Seja $\left\{a_{n}^{j}: n \in \omega\right\}$ enumeração crescente de $B_{j}, \forall j \in \omega$. De (5), segue-se que $a_{n}^{j}>h_{j}(n)$, $\forall j \in \omega$ e $\forall n \in \omega$. Assim, de (4) temos:

(6) $n<a_{n}^{j}$ e $a_{n}^{j} \in A_{n}^{j}, \forall j \in \omega$ e $\forall n \in \omega$.

Portanto, as sequências $\left\{a_{n}^{j}: n \in \omega\right\}, \forall j \in \omega$, satisfazem as condições do lema 1.2.6. Por esse lema, existe uma família $\left\{I_{j}: j \in \omega\right\}$ de subconjuntos de $\omega$ satisfazendo:

(7) $\left\{a_{k}^{j}: k \in I_{j}\right\} \in p_{j}, \forall j \in \omega$;

(8) $\left\{\left[k, a_{k}^{j}\right]: j \in \omega, k \in I_{j}\right\}$ são 2 a 2 disjuntos.

A propriedade (8) implica que $\left\{I_{j}: j \in \omega\right\}$ são 2 a 2 disjuntos. Seja $\left\{i_{m}: m \in \omega\right\}$ enumeração crescente de $\bigcup_{j<\omega} I_{j}$ e seja $r: \omega \longmapsto \omega$ tal que $r(m)=j$ se, e somente se, $i_{m} \in I_{j}$. A função $r$ está bem definida porque os $I_{j}$ 's são 2 a 2 disjuntos.

Defina $b_{m}:=a_{i_{m}}^{r(m)}$ e $E_{m}:=F_{i_{m}}$, para todo $m \in \omega$.

As propriedades (0) e (2) implicam que valem os itens (a) e (b), respectivamente. O item (e) segue da definição de $r$ e de (7). O item (d) também é satisfeito. De fato, temos que $b_{m}=a_{i_{m}}^{r(m)} \in A_{i_{m}}^{r(m)}$ para cada $m \in \omega$, pelo item (6). Pela definição de $A_{i_{m}}^{r(m)}$, a família $\left\{g_{\xi}\left(b_{m}\right): \xi \in E_{m} \cap S_{r(m)}\right\} \cup\left\{\{\mu\}: \mu \in E_{m}\right\}=\left\{g_{\xi}\left(b_{m}\right): \xi \in F_{i_{m}} \cap S_{r(m)}\right\} \cup\left\{\{\mu\}: \mu \in F_{i_{m}}\right\}$ é linearmente independente para cada $m \in \omega$.

Finalmente, checamos a condição (c). Por (8), $b_{m}=a_{i_{m}}^{r(m)} \leq i_{m+1}-1$ e $E_{m}=F_{i_{m}} \subseteq$ $F_{i_{m+1}-1}$, para todo $m \in \omega$. Assim, temos que $E_{m} \cup\left\{g_{\xi}\left(b_{m}\right): \xi \in E_{m}\right\}$ está contido em $F_{i_{m+1}-1} \cup \bigcup\left\{g_{\xi}(k): k \leq i_{m+1}-1, \xi \in F_{i_{m+1}-1}\right\} \subseteq F_{i_{m+1}}=E_{m+1}$, para cada $m \in \omega$. Logo, a condição (c) é também satisfeita e a prova está completa para os itens (a)-(e). 
Para o item (f), é suficiente na construção de $\left\{F_{i}: i \in \omega\right\}$ garantir que para cada $i \in \omega$, $\omega \times \alpha \cap F_{i}=(k+1) N \times \alpha$, já que $\alpha$ é finito, para algum $N \in \omega$. Isto é possível pois cada $F_{i}$ é finito. Os $E_{i}$ 's claramente satisfazem a condição (f).

Lema 4.2: Seja $\left\{p_{\xi}: \xi<\mathfrak{c}\right\}$ coleção de ultrafiltros seletivos incomparáveis. Sejam $F_{0}$, $F_{1}, \ldots, F_{k}, k+1$ subconjuntos finitos de $\mathfrak{c} \times k$ e $\left\{g_{\xi, i}: \xi<\mathfrak{c}\right.$ e $\left.i<k\right\} \subseteq\left([\mathfrak{c} \times k]^{<\omega}\right)^{\omega}$ tal que $\bigcup_{n \in \omega}^{i<k} g_{\xi, i}(n) \subseteq \xi \times k$, se $\xi>\omega$, ou $\bigcup_{n \in \omega}^{i<k} g_{\xi, i}(n) \subseteq \omega \times k$, caso contrário. Tome $g_{\xi, i}$ injetora $\forall \xi<\mathfrak{c}$ e $i<k$. Considere ainda a família $\left\{A_{\xi}: \xi<\mathfrak{c}\right\}$ de subconjuntos de $k$ tal que dado $\xi \in \mathfrak{c},\left\{\left[g_{\xi, i}\right]_{p_{\xi}}: i \in A_{\xi}\right\} \cup\left\{[\vec{\mu}]_{p_{\xi}}: \mu \in \mathfrak{c}\right\}$ é linearmente independente. Então, existe um homomorfismo $\phi:[\mathfrak{c} \times k]^{<\omega} \longmapsto\{0,1\}$ tal que:

(i) $\phi\left(F_{j}\right)=1$, se $j<k+1$ e $F_{j} \neq \emptyset$;

(ii) $\left\{n \in \omega: \phi\left(g_{\xi, i}(n)\right)=\phi(\{\xi\} \times\{i\})\right\} \in p_{\xi}, \forall i \in A_{\xi} \subseteq k, \forall \xi<\mathfrak{c}$;

(iii) $\{n \in \omega:(\phi(\{(k+1) n\} \times\{0\}), \phi(\{(k+1) n+1\} \times\{0\}), \ldots, \phi(\{(k+1) n+k\} \times\{0\}))$ $\left.=\left(\phi\left(F_{0}\right), \phi\left(F_{1}\right), \ldots, \phi\left(F_{k}\right)\right)\right\}$ é finito.

Demonstração: Seja $F=F_{0} \cup F_{1} \cup \ldots \cup F_{k}$. Existe um subconjunto enumerável $E$ de $\mathfrak{c} \times k$ tal que $F \cup(\omega \times k) \subseteq E$ e $g_{\xi, i}(n) \subseteq E, \forall(\xi, i) \in E$ e $n \in \omega$. Isto pode ser feito por indução: faça $S(\omega)=(\omega \times k)$ e supondo conhecido $S(\mu), \forall \mu<\xi$, seja $S(\xi)=(\{\xi\} \times k) \cup \bigcup_{(\{\mu\} \times k) \cap\left(\cup_{n \in \omega}^{i<k} g_{\xi, i}(n)\right) \neq \emptyset} S(\mu)$. Ao final, faça $E=\bigcup_{(\mu, i) \in F} S(\mu)$. Perceba que $E$ é construído de tal forma que $E=E^{\prime} \times k$, ou seja, se $(\xi, i) \in E$ então $(\xi, j) \in E, \forall j \in k$. Use o lema anterior para $\left\{p_{\xi}: \xi<E^{\prime}\right\}, F, E,\left\{g_{\xi, i}:(\xi, i) \in E\right\}$ e $\left\{S_{\xi}=\left\{(\xi, i): i \in A_{\xi}\right\}: \xi \in E^{\prime}\right\}$ para obter $\left\{b_{i}: i \in \omega\right\}, r: \omega \longmapsto E^{\prime}$ e $\left\{E_{i}: i \in \omega\right\}$ satisfazendo:

(0) $\forall i \in \omega, E_{i} \cap(\omega \times k)=(k+1) N \times k$, para algum $N \in \omega$;

(1) $F \subseteq E_{0}$;

(2) $E=\bigcup_{i \in \omega} E_{i}$ 
(3) $E_{i+1} \supseteq \bigcup\left\{g_{\xi, j}\left(b_{i}\right):(\xi, j) \in E_{i}\right\} \cup E_{i}, \forall i \in \omega$;

(4) $\left\{g_{r(i), j}\left(b_{i}\right): \forall j \in A_{r(i)}\right\} \cup\left\{\{(\mu, t)\}:(\mu, t) \in E_{i}\right\}$ é linearmente independente;

(5) $\left\{b_{i}: i \in r^{-1}(\xi)\right\} \in p_{\xi}, \forall \xi \in E^{\prime}$.

Definiremos primeiramente $\phi$ em $[E]^{<\omega}$. Isto é feito por indução no comprimento $\omega$. Para todo $j<k+1$ seja $\phi\left(F_{j}\right)=1$ se $F_{j} \neq \emptyset$ e estenda $\phi$ a um homomorfismo em $\left[E_{0}\right]^{<\omega}$. Propriedade (i) será satisfeita independentemente da forma que escolhamos estender o homomorfismo $\phi$. Queremos definir $\phi$ no subgrupo $\left[E_{n}\right]^{<\omega}, \forall n \in \omega$, de tal forma que:

(6) $\phi\left(g_{r(m), j}\left(b_{m}\right)\right)=\phi(\{r(m)\} \times\{j\}), \forall m<n$ e $j \in A_{r(m)}, \operatorname{com} A_{r(m)} \subseteq k$, se $r(m) \in E_{m}^{\prime}$;

(7) $(\phi(\{(k+1) t\} \times\{0\}), \phi(\{(k+1) t+1\} \times\{0\}), \ldots, \phi(\{(k+1) t+k\} \times\{0\})) \neq$ $\left(\phi\left(F_{0}\right), \phi\left(F_{1}\right), \ldots, \phi\left(F_{k}\right)\right), \forall m<n$ e $(k+1) t \in\left(E_{m+1}^{\prime} \backslash E_{m}^{\prime}\right) \cap \omega$.

Condições (6) e (7) são trivialmente satisfeitas para $n=0$. Suponha que são satisfeitas para $n$. Provaremos que o homomorfismo $\phi$ pode ser estendido a $\left[E_{n+1}\right]^{<\omega}$ de forma que (6) e (7) valem para $n+1$.

Pelo item (4), $V=\left\{g_{r(n), j}\left(b_{n}\right): j \in A_{r(n)}\right\} \cup\left\{\{(\mu, q)\}:(\mu, q) \in E_{n}\right\}$ é conjunto linearmente independente. Chamemos $C=\left\{\{(\mu, q)\}:(\mu, q) \in E_{n}\right\}$ e $\left.W_{t}=\{\{(k+1) t\} \times\{0\}),\{(k+1) t+1\} \times\{0\}, \ldots,\{(k+1) t+k\} \times\{0\}\right\}$. Afirmamos que para todo $t \in B_{n}=\left\{m \in \omega:(k+1) m \in E_{n+1}^{\prime} \backslash E_{n}^{\prime}\right\}$, podemos tomar $s_{t} \in k$ tal que $\left\{g_{r(n), j}\left(b_{n}\right): j \in A_{r(n)}\right\} \cup\left\{\left\{(k+1) t+s_{t}\right\} \times\{0\}: t \in B_{n}\right\} \cup C$ é linearmente independente. Primeiramente, temos que se $t \in B_{n}$, então $W_{t} \subseteq E_{n+1} \backslash E_{n}$, pois $E_{m}^{\prime} \cap \omega=(k+1) N, \forall m \in \omega(N \in \omega)$. Assim, obtemos o conjunto $\{\{(k+1) t\} \times\{0\})$, $\left.\left.\{(k+1) t+1\} \times\{0\}, \ldots,\{(k+1) t+k\} \times\{0\}: t \in B_{n}\right\}\right\} \cup C$ que é linearmente independente. Se $B_{n}=\left\{t_{0}, t_{1}, . ., t_{j}\right\}$, como a dimensão de $W_{t_{0}}$ é maior do que $\left|A_{r(n)}\right|$, existe $s_{t_{0}} \in k+1$ tal que $V_{t_{0}}=\left\{g_{r(n), j}\left(b_{n}\right): j \in A_{r(n)}\right\} \cup\left\{\left\{(k+1) t+s_{t_{0}}\right\} \times\{0\}\right\} \cup C$ é linearmente independente. Para o próximo passo, considere o conjunto l.i. $W_{t_{1}} \cup\left\{\left\{(k+1) t+s_{t_{0}}\right\} \times\{0\}\right\} \cup C$. 
Novamente, como a dimensão de $W_{t_{1}}$ é maior do que $\left|A_{r(n)}\right|$, existe $s_{t_{1}} \in k+1$ tal que $V_{t_{1}}=\left\{g_{r(n), j}\left(b_{n}\right): j \in A_{r(n)}\right\} \cup\left\{\left\{(k+1) t+s_{t_{1}}\right\} \times\{0\}\right\} \cup\left\{\left\{(k+1) t+s_{t_{0}}\right\} \times\{0\}\right\} \cup C$ é linearmente independente. Após um número finito de passos, encontramos $V_{t_{j}}$, o conjunto da afirmação.

Pelo item (3), podemos estender $\left.\phi\right|_{\left[E_{n}\right]<\omega}$ a $\left[E_{n+1}\right]^{<\omega}$ tal que $\phi\left(g_{r(n), j}\left(b_{n}\right)\right)=\phi(\{r(n)\} \times\{j\}), \forall j \in A_{r(n)}-\operatorname{caso} r(n) \in E_{n}^{\prime}$, e $\phi\left(\left\{(k+1) t+s_{t}\right\} \times\{0\}\right)=$ $1-\phi\left(F_{s_{t}}\right)$. Portanto, (6) e (7) são satisfeitas para $n+1$.

A propriedade (2) e a definição de $E$ implicam que se $t \in \omega \backslash E_{0}^{\prime}$, então existe $n \in \omega$ tal que $(k+1) t \in E_{n+1}^{\prime} \backslash E_{n}^{\prime}$. A propriedade (7) implica então em

$$
\{t \in \omega:(\phi(\{(k+1) t\} \times\{0\}), \phi(\{(k+1) t+1\} \times\{0\}), \ldots, \phi(\{(k+1) t+k\} \times\{0\}))
$$

$\left.=\left(\phi\left(F_{0}\right), \phi\left(F_{1}\right), \ldots, \phi\left(F_{k}\right)\right)\right\} \subseteq E_{0}^{\prime}$, que é finito. Assim, qualquer extensão do homomorfismo $\phi$ a $[\mathfrak{c} \times k]^{<\omega}$ satisfaz (iii).

Afirmamos que $\phi$ satisfaz a condição (ii) para todo $(\xi, j) \in E$. De fato, por (2), dado $(\xi, j) \in E, \exists n \in \omega$ tal que $(\xi, j) \in E_{n+1} \backslash E_{n}$. Considere inicialmente $j \in A_{\xi}$. Por (6), $\phi(\{\xi\} \times\{j\})=\phi\left(g_{\xi, j}\left(b_{m}\right)\right), \forall m \in r^{-1}(\xi) \backslash n+1$, pois $\xi \in E_{n+1}^{\prime}$. Como $r^{-1}(\xi) \backslash n+1 \in p_{\xi}$ temos que $(\xi, j)$ satisfaz a condição (ii). Caso $j \notin A_{\xi}$, como $g_{\xi, j}\left(b_{n}\right)$ é combinação linear finita de elementos em $\left\{g_{\xi, i}\left(b_{n}\right): i \in A_{\xi}\right\} \cup\left\{\{(\mu, q)\}:(\mu, q) \in E_{n}\right\}$, temos que $\left\{g_{\xi, j}\left(b_{n}\right): n \in \omega\right\}$ possui $p_{\xi}$ - limite: se $g_{\xi, j}\left(b_{n}\right)=\triangle\left(\left\{g_{\xi, i}\left(b_{n}\right): i \in L_{1}\right\} \cup\left\{\{(\mu, q)\}:(\mu, q) \in L_{2}\right\}\right), L_{1}$ e $L_{2}$ subconjuntos finitos de $A_{\xi}$ e $E_{n}$, respectivamente, então

$$
\begin{aligned}
& \phi\left(g_{\xi, j}\left(b_{n}\right)\right)=\sum\left(\left\{\phi\left(g_{\xi, i}\left(b_{n}\right)\right): i \in L_{1}\right\}+\left\{\phi(\{(\mu, q)\}):(\mu, q) \in L_{2}\right\}\right) . \\
& \text { Daí, } \\
& \left\{n \in \omega: \phi\left(g_{\xi, j}\left(b_{n}\right)\right)=\left(\sum\left(\left\{\phi(\{\xi\} \times\{i\}): i \in L_{1}\right\}\right)+\sum\left(\left\{\phi(\{(\mu, q)\}):(\mu, q) \in L_{2}\right\}\right)\right)\right\}
\end{aligned}
$$
contém o conjunto $\bigcap\left\{n \in \omega: \phi\left(g_{\xi, i}\left(b_{n}\right)\right)=\phi(\{\xi\} \times\{i\}), \forall i \in L_{1}\right\}$. Como a última intersecção de elementos de $p_{\xi}$ é finita e $p_{\xi}$ é filtro, temos que o primeiro conjunto pertence a $p_{\xi}$ e seguese que $\left\{g_{\xi, j}\left(b_{n}\right): n \in \omega\right\}$ possui $p_{\xi}$ - limite.

A construção estará terminada se estendermos $\left.\phi\right|_{[E]<\omega}$ a $[\mathfrak{c} \times k]^{<\omega}$ tal que (ii) é satisfeita 
para cada $(\xi, t) \in(\mathfrak{c} \times k) \backslash E$, com $t \in A_{\xi}$. Por indução em $\mathfrak{c} \backslash E^{\prime}$, definiremos $\phi$ em $[E \cup(\xi, t)]^{<\omega}$ tal que (ii) é satisfeita para cada $(\xi, t) \in(\mathfrak{c} \times k) \backslash E, t \in A_{\xi}$. Seja $\gamma<\mathfrak{c}$ o menor ordinal para o qual $\phi(\{(\gamma, t)\})$, com $t \in k$, ainda não foi definido. Temos então que $\forall t \in A_{\gamma}, \phi(\{(\gamma, t)\})$ não está definido e, por outro lado, $\left.\phi\right|_{[E \cup(\gamma, t)]<\omega}$ está determinado e pela definição de $g_{\gamma, t}$, a sequência $\left\{\phi\left(g_{\gamma, t}(k)\right): k \in \omega\right\}$ já está também determinada. O conjunto $\left\{(\gamma, t): t \in A_{\gamma}\right\} \cup\{(\xi, s): \xi \in \gamma\}$ é linearmente independente, logo usando a $p_{\gamma^{-}}$ compacidade de $\{0,1\}$, podemos fazer $\phi(\{(\gamma, t)\})=p_{\gamma}-\lim \left\{\phi\left(g_{\gamma, t}(k)\right): k \in \omega\right\}, \forall t \in A_{\gamma}$. O homomorfismo $\phi$ satisfaz (ii) para cada $(\xi, t)$, com $\xi \in \gamma+1$ e $t \in A_{\xi}$.

Estamos prontos para construir um grupo $G$ enumeravelmente compacto tal que $G^{k}$ é enumeravelmente compacto, mas $G^{k+1}$ não é:

Exemplo 4.1: Suponha que existe uma família $\left\{p_{\xi}: \xi<\mathfrak{c}\right\}$ de ultrafiltros seletivos incomparáveis. Então existe um grupo $G$ enumeravelmente compacto tal que $G^{k}$ é enumeravelmente compacto, mas $G^{k+1}$ não é.

Demonstração: Seja $\left\{g_{\xi, i}: \xi<\mathfrak{c}\right.$ e $\left.i<k\right\} \subseteq\left([\mathfrak{c} \times k]^{<\omega}\right)^{\omega}$ tal que:

(1) $\bigcup_{n \in \omega}^{i<k} g_{\xi, i}(n) \subseteq \xi \times k$, se $\xi>\omega$, ou $\bigcup_{n \in \omega}^{i<k} g_{\xi, i}(n) \subseteq \omega \times k$, caso contrário;

(2) $g_{\xi, i}$ é injetora $\forall \xi<\mathfrak{c}$ e $i<k$, e para todo $g \in\left([\mathfrak{c} \times k]^{<\omega}\right)^{\omega}$ função injetora, existe $(\mu, t) \in \mathfrak{c} \times k$ tal que $g=g_{\mu, t}$.

Seja $\mathcal{C}=\left([\mathfrak{c} \times k]^{<\omega}\right)^{k+1}$. O conjunto $\mathcal{C}$ indexará a família de homomorfismos que precisaremos. Use o lema anterior para a sequência $\left\{g_{\xi, i}: \xi<\mathfrak{c}\right.$ e $\left.i<k\right\}$ e para cada k+1- upla $\left(F_{0}, F_{1}, \ldots, F_{k}\right)$ de forma a obter um homomorfismo $\phi_{\left(F_{0}, F_{1}, \ldots, F_{k}\right)}:[\mathfrak{c} \times k]<\omega \longmapsto 2$ satisfazendo:

(3) $\phi_{\left(F_{0}, F_{1}, \ldots, F_{k}\right)}\left(F_{0}\right)=1$ se $F_{0} \neq \emptyset$;

(4) $\left\{n \in \omega: \phi_{\left(F_{0}, F_{1}, \ldots, F_{k}\right)}\left(g_{\xi, i}(n)\right)=\phi_{\left(F_{0}, F_{1}, \ldots, F_{k}\right)}(\{\xi\} \times\{i\})\right\} \in p_{\xi}, \forall i \in A_{\xi} \subseteq k, \forall \xi<\mathfrak{c}$; 
(5) $\left\{n \in \omega:\left(\phi_{\left(F_{0}, F_{1}, \ldots, F_{k}\right)}(\{(k+1) n\} \times\{0\}), \phi_{\left(F_{0}, F_{1}, \ldots, F_{k}\right)}(\{(k+1) n+1\} \times\{0\}), \ldots\right.\right.$, $\left.\left.\phi_{\left(F_{0}, F_{1}, \ldots, F_{k}\right)}(\{(k+1) n+k\} \times\{0\})\right)=\left(\phi_{\left(F_{0}, F_{1}, \ldots, F_{k}\right)}\left(F_{0}\right), \phi_{\left(F_{0}, F_{1}, \ldots, F_{k}\right)}\left(F_{1}\right), \ldots, \phi_{\left(F_{0}, F_{1}, \ldots, F_{k}\right)}\left(F_{k}\right)\right)\right\}$ é finito.

Seja $\mathcal{H}:[\mathfrak{c} \times k]^{<\omega} \longmapsto 2^{\mathcal{C}}$ a função diagonal $\triangle_{\left(F_{0}, F_{1}, \ldots, F_{k}\right) \in \mathcal{C}} \phi_{\left(F_{0}, F_{1}, \ldots, F_{k}\right)}$ definida por $\pi_{\left(F_{0}, F_{1}, \ldots, F_{k}\right)} \circ \mathcal{H}=\phi_{\left(F_{0}, F_{1}, \ldots, F_{k}\right)}$ para cada $\left(F_{0}, F_{1}, \ldots, F_{k}\right) \in \mathcal{C}$, onde $\pi_{\left(F_{0}, F_{1}, \ldots, F_{k}\right)}: 2^{\mathcal{C}} \longmapsto 2$ é a projeção na coordenada $\left(F_{0}, F_{1}, \ldots, F_{k}\right)$.

Defina $G:=\mathcal{H}\left([\mathfrak{c} \times k]^{<\omega}\right)$ com a topologia de subespaço. Afirmamos que:

(6) O homomorfismo $\mathcal{H}$ é uma imersão.

De fato, para todo $F$ subconjunto finito e não-vazio de $[\mathfrak{c} \times k]^{<\omega}$, a k+1-upla $(F, F, \ldots, F)$ está em $\mathcal{C}$. Pelo item $(3), \phi_{(F, F, \ldots, F)}(F) \neq 0$. Portanto, $\mathcal{H}(F) \neq 0$.

(7) $G^{k}$ é enumeravelmente compacto.

É suficiente demonstrar que cada sequência 1-1 em $G^{k}$ tem ponto de acumulação. Seja $\left\{\left(a_{0}(n), a_{1}(n), \ldots, a_{k-1}(n)\right): n \in \omega\right\}$ seqüência 1-1 em $G^{k}=\left(\mathcal{H}\left([\mathfrak{c} \times k]^{<\omega}\right)\right)^{k}$ tal que $a_{j}(n)=\mathcal{H}\left(F_{j}^{n}\right), \forall n \in \omega, j \in k$. Pela construção de $\left\{g_{\xi, i}: \xi<\mathfrak{c}\right.$ e $\left.i<k\right\} \subseteq\left([\mathfrak{c} \times k]^{<\omega}\right)^{\omega}$, existe $\xi<\mathfrak{c}$ tal que $g_{\xi, j}(n)=F_{j}^{n}, \forall n \in \omega, j \in k$. A sequência pode então ser vista como $\left\{\left(\mathcal{H}\left(g_{\xi, 0}(n)\right), \mathcal{H}\left(g_{\xi, 1}(n)\right), \ldots, \mathcal{H}\left(g_{\xi, k-1}(n)\right)\right): n \in \omega\right\}$. Se $\xi \in \mathfrak{c}$ e $\left(F_{0}, F_{1}, \ldots, F_{k}\right) \in \mathcal{C}$ então $\phi_{\left(F_{0}, F_{1}, \ldots, F_{k}\right)}(\{\xi\} \times\{i\})=p_{\xi}-\lim \left\{\phi_{\left(F_{0}, F_{1}, \ldots, F_{k}\right)}\left(g_{\xi, i}(n): n \in \omega\right\} \forall i \in A_{\xi}\right.$, pelo item (4). Logo, pelo lema 1.1.5, $\mathcal{H}(\{\xi\} \times\{i\})=p_{\xi}-\lim \left\{\mathcal{H}\left(g_{\xi, i}(n): n \in \omega\right\}, \forall i \in A_{\xi}\right.$. Se $l \notin A_{\xi}$, temos que existe $\Omega$ elemento de $p_{\xi}$ tal que $g_{\xi, l}(n)=\triangle\left(\left\{g_{\xi, i}(n): i \in A_{\xi, l}\right\}\right) \triangle F$, $\forall n \in \Omega, \operatorname{com} F \in[\mathfrak{c} \times k]^{<\omega}$ e $A_{\xi, l} \subseteq A_{\xi}$. Se $\left(F_{0}, F_{1}, \ldots, F_{k}\right) \in \mathcal{C}, \operatorname{como} \phi_{\left(F_{0}, F_{1}, \ldots, F_{k}\right)}$ é homomorfismo, $\phi_{\left(F_{0}, F_{1}, \ldots, F_{k}\right)}\left(g_{\xi, l}(n)\right)=\sum\left(\left\{\phi_{\left(F_{0}, F_{1}, \ldots, F_{k}\right)}\left(g_{\xi, i}(n)\right): i \in A_{\xi, l}\right\}\right)+\phi_{\left(F_{0}, F_{1}, \ldots, F_{k}\right)}(F)$, $\forall n \in \Omega$. Assim, $\mathcal{H}\left(g_{\xi, l}(n)\right)=\sum\left(\left\{\mathcal{H}\left(g_{\xi, i}(n)\right): i \in A_{\xi, l}\right\}\right)+\mathcal{H}(F), \forall n \in \Omega$. Daí, pelo lema 1.1.4 temos que se $\left\{\sum\left(\left\{\mathcal{H}\left(g_{\xi, i}(n)\right): i \in A_{\xi, l}\right\}\right)+\mathcal{H}(F): n \in \omega\right\}$ possui $p_{\xi}$-limite, então a sequência $\left\{\mathcal{H}\left(g_{\xi, l}(n): n \in \omega\right\}\right.$ também possui e os $p_{\xi}$-limites coincidem. Como $A_{\xi, l}$ é finito, 
temos que a soma dos $p_{\xi}$-limites de $\left\{\mathcal{H}\left(g_{\xi, i}(n)\right): n \in \omega\right\}, i \in A_{\xi, l}$, e $\{\mathcal{H}(F): n \in \omega\}$ é igual ao $p_{\xi}$-limite da soma $\left\{\sum\left(\left\{\mathcal{H}\left(g_{\xi, i}(n)\right): i \in A_{\xi, l}\right\}\right)+\mathcal{H}(F): n \in \omega\right\}$. Assim, $\sum\left(\left\{\mathcal{H}(\{\xi\} \times\{i\}): i \in A_{\xi, l}\right\}\right)+\mathcal{H}(F)$ é $p_{\xi}$-limite para a sequência $\left\{\mathcal{H}\left(g_{\xi, l}(n): n \in \omega\right\}\right.$, $l \notin A_{\xi}$. Logo, a sequência $\left\{\left(\mathcal{H}\left(g_{\xi, 0}(n)\right), \mathcal{H}\left(g_{\xi, 1}(n)\right), \ldots, \mathcal{H}\left(g_{\xi, k-1}(n)\right)\right): n \in \omega\right\}$ possui ponto de acumulação e $G^{k}$ é enumeravelmente compacto.

(8) $G^{k+1}$ não é enumeravelmente compacto.

Afirmamos que a seqüência

$$
\{(\mathcal{H}(\{(k+1) n\} \times\{0\}), \mathcal{H}(\{(k+1) n+1\} \times\{0\}), \ldots, \mathcal{H}(\{(k+1) n+k\} \times\{0\})): n \in \omega\}
$$

não possui ponto de acumulação em $G^{k+1}$. Seja $\left(a_{0}, a_{1}, \ldots, a_{k}\right)$ um elemento arbitrário de $G^{k+1}$. Existe $\left(F_{0}, F_{1}, \ldots, F_{k}\right) \in \mathcal{C}$ tal que $a_{j}=\mathcal{H}\left(F_{j}\right)$ para $j<k+1$. Segue-se do item (5) que $\left\{n \in \omega: \pi_{\left(F_{0}, F_{1}, \ldots, F_{k}\right)} \circ \mathcal{H}\left(\left\{(\{(k+1) n+j\} \times\{0\})=\pi_{\left(F_{0}, F_{1}, \ldots, F_{k}\right)}\left(a_{j}\right), j<k+1\right\}\right.\right.$ pode ser visto como o conjunto de $n \in \omega$ tais que

$$
\begin{aligned}
& \left(\phi_{\left(F_{0}, F_{1}, \ldots, F_{k}\right)}(\{(k+1) n\} \times\{0\}), \ldots, \phi_{\left(F_{0}, F_{1}, \ldots, F_{k}\right)}(\{(k+1) n+k\} \times\{0\})\right)= \\
& \left.=\left(\phi_{\left(F_{0}, F_{1}, \ldots, F_{k}\right)}\left(F_{0}\right), \phi_{\left(F_{0}, F_{1}, \ldots, F_{k}\right)}\left(F_{1}\right), \ldots, \phi_{\left(F_{0}, F_{1}, \ldots, F_{k}\right)}\left(F_{k}\right)\right)\right\}, q u e \text { é finito. }
\end{aligned}
$$

Portanto, $\left(a_{0}, a_{1}, \ldots, a_{k}\right)$ não é ponto de acumulação da seqüência

$$
\{(\mathcal{H}(\{(k+1) n\} \times\{0\}), \mathcal{H}(\{(k+1) n+1\} \times\{0\}), \ldots, \mathcal{H}(\{(k+1) n+k\} \times\{0\})): n \in \omega\}
$$
em $G^{k+1}$. 


\section{Capítulo 5}

\section{Produto infinito de grupos}

\section{enumeravelmente compactos.}

Apresentaremos neste capítulos duas construções que servirão de contra-exemplos para a produtividade da compacidade enumerável em um produto infinito de um mesmo grupo topológico enumeravelmente compacto. Os primeiros contra-exemplos para o caso infinito foram obtidos por Tomita em [9]. Aqui, apresentaremos novas construções para esses contraexemplos, em uma tentativa de fazer algo semelhante ao que foi feito para o caso quadrado no capítulo 3 e do caso finito feito no capítulo anterior. Na secção 5.2, construiremos um grupo topológico enumeravelmente compacto $H$ tal que $H^{\gamma}$ é enumeravelmente compacto para todo $\gamma<2^{\mathfrak{c}}$, mas $H^{2^{\mathfrak{c}}}$ não é. Na secção 5.3, construiremos um grupo topológico enumeravelmente compacto $H$ tal que $H^{\gamma}$ é enumeravelmente compacto para todo $\gamma<\alpha$, mas $H^{\alpha}$ não é enumeravelmente compacto $\left(\omega \leq \alpha<2^{\mathfrak{c}}\right)$. 


\subsection{Preliminares.}

Aqui, demonstraremos dois lemas que serão usados nas construções das secções seguintes.

Lema 5.1.1: Seja $\left\{p_{\xi}: \omega \leq \xi<2^{\mathfrak{c}}\right\}$ uma coleção de ultrafiltros seletivos incomparáveis. Seja $\mathcal{F}=\left\{g_{\xi, \beta}: \omega \leq \xi<2^{\mathfrak{c}} \wedge \beta \leq \gamma<\alpha\right\}$ uma enumeração de todas as funções injetoras em $\left(\left[2^{\mathfrak{c}} \times \alpha\right]^{<\omega}\right)^{\omega}$, com $\alpha \leq 2^{\mathfrak{c}}$, tais que $\bigcup_{n \in \omega}^{\beta \leq \gamma} g_{\xi, \beta}(n) \subseteq \xi \times \alpha$, para todo ordinal infinito $\xi<2^{\mathfrak{c}}$. Considere ainda a família $\left\{A_{\xi}: \omega \leq \xi<2^{\mathfrak{c}}\right\}$ de subconjuntos de $\alpha$ tal que dado $\xi \in 2^{\mathfrak{c}} \backslash \omega,\left\{\left[g_{\xi, \beta}\right]_{p_{\xi}}: \beta \in A_{\xi}\right\} \cup\left\{[\vec{\mu}]_{p_{\xi}}: \mu \in 2^{\mathfrak{c}}\right\}$ é linearmente independente. Então, dado $F \in\left[2^{\mathfrak{c}} \times \alpha\right]^{<\omega}$, existe um homomorfismo $\phi:\left[2^{\mathfrak{c}} \times \alpha\right]^{<\omega} \longmapsto\{0,1\}$ tal que:

(i) $\phi(F)=1$, se $F \in\left[2^{\mathfrak{c}} \times \alpha\right]^{<\omega} \backslash \emptyset$;

(ii) $\left\{n \in \omega: \phi\left(g_{\xi, \beta}(n)\right)=\phi(\{\xi\} \times\{\beta\})\right\} \in p_{\xi}, \forall \beta \in A_{\xi} \subseteq \alpha, \operatorname{com} \omega \leq \xi<2^{\mathfrak{c}}$;

Demonstração: Pode-se demonstrar por indução que existe um subconjunto enumerável $E$ de $2^{\mathfrak{c}} \times \alpha$ tal que $F \subseteq E$ e $g_{\xi, \beta}(n) \subseteq E, \forall(\xi, \beta) \in E$ e $n \in \omega$. De fato, começamos por definir $S(\omega, \beta)=\{(\omega, \beta)\} \cup \bigcup_{n \in \omega} g_{\omega, \beta}(n), \forall \beta \in \alpha$. Supondo $S(\xi, \beta)$ definido $\forall \beta \in \alpha$, fazemos $S(\xi+1, \beta)=\{(\xi+1, \beta)\} \cup \bigcup_{(\mu, \gamma) \in \bigcup_{n \in \omega}} g_{\xi+1, \beta}(n) S(\mu, \gamma)$. Faça, então, $E=\bigcup_{(\mu, \gamma) \in F} S(\mu, \gamma)$

Use o lema 4.1 para $\left\{p_{\xi}: \xi \in E^{\prime}\right\}, F, E,\left\{g_{\xi, \beta}:(\xi, \beta) \in E\right\}$ e $\left\{S_{\xi}=\left\{(\xi, \beta): \beta \in A_{\xi}\right\}: \xi \in E^{\prime}\right\}$ para obter $\left\{b_{i}: i \in \omega\right\}, r: \omega \longmapsto E^{\prime}$ e $\left\{E_{i}: i \in \omega\right\}$ satisfazendo:

(1) $F \subseteq E_{0}$;

(2) $E=\bigcup_{i \in \omega} E_{i}$;

(3) $E_{i+1} \supseteq \bigcup\left\{g_{\xi, \beta}\left(b_{i}\right):(\xi, \beta) \in E_{i}\right\} \cup E_{i}, \forall i \in \omega$; 
(4) $\left\{g_{r(i), \beta}\left(b_{i}\right), \forall \beta \in S_{r(i)} \cap E_{i}\right\} \cup\left\{\{(\mu, t)\}:(\mu, t) \in E_{i}\right\}$ é linearmente independente, $\forall i \in \omega$;

(5) $\left\{b_{i}: i \in r^{-1}(\xi)\right\} \in p_{\xi}, \forall \xi \in E^{\prime}$.

Definiremos primeiramente $\phi$ em $[E]^{<\omega}$. Isto é feito por indução no comprimento $\omega$. Comece por determinar $\phi(F)=1$, se $F \neq \emptyset$, e estenda $\phi$ a um homomorfismo qualquer em $\left[E_{0}\right]^{<\omega}$. A propriedade (i) será satisfeita independentemente da forma que vamos estender o homomorfismo $\phi$. Queremos definir $\phi$ no subgrupo $\left[E_{n}\right]^{<\omega}, \forall n \in \omega$, de tal forma que:

(6) $\phi\left(g_{r(m), \beta}\left(b_{m}\right)\right)=\phi(\{r(m)\} \times\{\beta\}), \forall m<n$ e $(r(m), \beta) \in S_{r(m)} \cap E_{m}$.

A condição (6) é trivialmente satisfeita para $n=0$. Suponha que é satisfeita para $n$. Provaremos que o homomorfismo $\phi$ pode ser estendido a $\left[E_{n+1}\right]^{<\omega}$ de forma que (6) vale para $n+1$.

Pelo item (4), se $r(n) \in E_{n}^{\prime}$ temos que $\left\{g_{r(n), \beta}\left(b_{n}\right): \beta \in S_{r(n)} \cap E_{n}\right\} \cup$ $\cup\left\{\{(\mu, q)\}:(\mu, q) \in E_{n}\right\}$ é linearmente independente. Por hipótese, $\phi(\{r(n)\} \times\{\beta\})$ já está definido $\forall(r(n), \beta) \in E_{n}$, logo pelo item (3) podemos estender $\phi$ a $E_{n+1}$ de tal forma que $\phi\left(g_{r(n), \beta}\left(b_{n}\right)\right)=\phi(\{r(n)\} \times\{\beta\}), \forall \beta \in A_{r(n)}$. Assim, vale o item (6) também para $n+1$. Pelo item (2), no estágio $\omega, \phi$ estará definido em $[E]^{<\omega}$.

Afirmamos que $\phi$ satisfaz a condição (ii) para todo $(\xi, \beta) \in E, \operatorname{com} \beta \in A_{\xi}$. De fato, por (2), dado $(\xi, \beta) \in E, \exists n \in \omega$ tal que $(\xi, \beta) \in E_{n+1} \backslash E_{n}$. Por $(6), \phi(\{\xi\} \times\{\beta\})=\phi\left(g_{\xi, \beta}\left(b_{m}\right)\right)$, $\forall m \in r^{-1}(\xi) \backslash n+1$, pois $\xi=r(m) \in E_{n+1}^{\prime}$. Como $r^{-1}(\xi) \backslash n+1 \in p_{\xi}$ temos que $(\xi, \beta)$ satisfaz a condição (ii).

A construção estará terminada se estendermos $\left.\phi\right|_{[E]^{<\omega}}$ a $\left[2^{\mathfrak{c}} \times \alpha\right]^{<\omega}$ tal que (ii) é satisfeita para cada $(\xi, \beta) \in\left(2^{\mathfrak{c}} \times \alpha\right) \backslash E$, com $\beta \in A_{\xi}$. Por indução em $2^{\mathfrak{c}} \backslash E^{\prime}$, definiremos $\phi$ em $[E \cup(\xi, \beta)]^{<\omega}$ de tal forma que (ii) esteja satisfeita para cada $(\xi, \beta) \in\left(2^{\mathfrak{c}} \times \alpha\right) \backslash E, \beta \in A_{\xi}$. Seja $\gamma<2^{\mathfrak{c}}$ o menor ordinal para o qual existe algum $\beta \in \alpha$ tal que $\phi(\{(\gamma, \beta)\})$ ainda não 
foi definido. Temos então que $\phi$ está determinado em $[E \cup\{(\eta, \lambda): \eta<\gamma \wedge \lambda<\beta\}]^{<\omega}$ e pela definição de $g_{\gamma, \beta}$, a sequência $\left\{\phi\left(g_{\gamma, \beta}(k)\right): k \in \omega\right\}$ estará então determinada. O conjunto $\left\{(\gamma, \beta): \beta \in A_{\gamma}\right\} \cup\{(\xi, s): \xi \in \gamma, s \in \alpha\}$ é linearmente independente, logo usando a $p_{\gamma}$-compacidade de $\{0,1\}$, podemos fazer $\phi(\{(\gamma, \beta)\})=p_{\gamma}-\lim \left\{\phi\left(g_{\gamma, \beta}(k)\right): k \in \omega\right\}$, $\forall \beta \in A_{\gamma}$. Se $\beta \in \alpha \backslash A_{\gamma}$, então definimos $\phi(\{(\gamma, \beta)\})$ de forma arbitrária. O homomorfismo $\phi$ satisfaz (ii) para cada $(\xi, \beta), \operatorname{com} \xi \in \gamma+1$ e $\beta \in A_{\xi}$.

Lema 5.1.2: Sejam $\left\{p_{\xi}: \omega \leq \xi<2^{\mathfrak{c}}\right\}, \alpha \leq 2^{\mathfrak{c}},\left\{g_{\xi, \beta}: \omega \leq \xi<2^{\mathfrak{c}}\right.$ e $\left.\beta \leq \gamma<\alpha\right\}$ e $\left\{A_{\xi}: \omega \leq \xi<2^{\mathfrak{c}}\right\}$ como no lema 5.1.1. Então, existe uma família $\left\{x_{\xi, \beta}: \xi<2^{\mathfrak{c}}, \beta \in \alpha\right\} \subseteq 2^{2^{c}}$ satisfazendo:

(I) $x_{\xi, \beta}$ é o $p_{\xi^{-}}$limite de $\left\{x_{g_{\xi, \beta}(n)}: n \in \omega\right\}$ para todo $\xi \in\left[\omega, 2^{\mathfrak{c}}\right)$, com $\beta \in A_{\xi}$;

(I-a) Se $\lambda \notin A_{\xi}$, a sequência $\left\{x_{g_{\xi, \lambda}(n)}: n \in \omega\right\}$ possui $\mathrm{p}_{\xi}$-limite em $\left\langle\left\{x_{\eta, \theta}:(\eta, \theta) \in \bigcup_{n \in \omega}^{\beta \leq \gamma} g_{\xi, \beta}(n) \cup\{\xi\} \times A_{\xi}\right\}\right\rangle$.

(II) para todo $A \subseteq \omega \times \alpha$, existe $(\eta, \beta) \in 2^{\mathfrak{c}} \times \alpha$ tal que $\left\{(n, \gamma) \in \omega \times \alpha: x_{n, \gamma}(\eta, \beta)=1\right\}=A ;$

(III) se $p, q \in \omega^{*}, p \neq q$, então para todo $\beta_{1}, \gamma_{1}, \beta_{2}, \gamma_{2} \in \alpha$, com $\beta_{i} \neq \gamma_{i} \forall i<2$, temos $p-\lim \left\{x_{n, \beta_{1}}+x_{n, \gamma_{1}}: n \in \omega\right\} \neq q-\lim \left\{x_{n, \beta_{2}}+x_{n, \gamma_{2}}: n \in \omega\right\}$ e $p-\lim \left\{x_{n, \beta}: n \in \omega\right\} \neq$ $p-\lim \left\{x_{n, \gamma}: n \in \omega\right\}$, se $\beta \neq \gamma$.

Demonstração: Começamos a demonstração provando que se $A \subseteq \omega \times \alpha$, então existe um homomorfismo $\psi_{A}:\left[2^{\mathfrak{c}} \times \alpha\right]^{<\omega} \longmapsto\{0,1\}$ tal que:

(a) $\left\{(n, \gamma) \in \omega \times \alpha: \psi_{A}(\{n\} \times\{\gamma\})=1\right\}=A$;

(b) $\left\{n \in \omega: \psi_{A}\left(g_{\xi, \beta}(n)\right)=\psi_{A}(\{\xi\} \times\{\beta\})\right\} \in p_{\xi}, \forall \beta \in A_{\xi} \subseteq \alpha, \omega \leq \xi<2^{\mathfrak{c}}$.

Sabemos que o conjunto $\{\{(n, \gamma)\}:(n, \gamma) \in \omega \times \alpha\}$ é linearmente independente. Assim, dado $A \subseteq \omega \times \alpha$, podemos definir um homomorfismo $\psi_{A}:\left[2^{\mathfrak{c}} \times \alpha\right]^{<\omega} \longmapsto\{0,1\}$ tal que $\left\{(n, \gamma) \in \omega \times \alpha: \psi_{A}(\{n\} \times\{\gamma\})=1\right\}=A$. Seja $\mu<2^{\mathfrak{c}}$ o menor ordinal para o 
qual $\psi_{A}(\{(\mu, \beta)\})$, com $\beta \in \alpha$, ainda não foi definido. Temos então que $\mu \geq \omega$ e que $\forall \beta \in A_{\mu}, \psi_{A}(\{(\mu, \beta)\})$ não está definido. Por outro lado, $\left.\psi_{A}\right|_{[E \cup(\mu, \beta)]<\omega}$ está determinado e pela definição de $g_{\mu, \beta}$, a sequência $\left\{\psi_{A}\left(g_{\mu, \beta}(k)\right): k \in \omega\right\}$ está também determinada. O conjunto $\{(\mu, \beta): \beta \in \alpha\} \cup\{(\xi, s): \xi \in \mu, s \in \alpha\}$ é linearmente independente, logo usando a $p_{\mu}$-compacidade de $\{0,1\}$, podemos fazer $\psi_{A}(\{(\mu, \beta)\})=p_{\mu}-\lim \left\{\psi_{A}\left(g_{\mu, \beta}(k)\right): k \in \omega\right\}$, $\forall \beta \in A_{\mu}$. Se $\beta \in \alpha \backslash A_{\mu}$, podemos definir $\psi_{A}(\{(\mu, \beta)\})$ de forma qualquer. O homomorfismo $\phi$ satisfaz $\left\{n \in \omega: \psi_{A}\left(g_{\xi, \beta}(n)\right)=\psi_{A}(\{\xi\} \times\{\beta\})\right\} \in p_{\xi}$ para cada $(\xi, \beta)$, com $\xi \in \mu+1 \mathrm{e}$ $\beta \in A_{\xi}$. Logo, por indução, podemos estender este homomorfismo a $\left[2^{\mathfrak{c}} \times \alpha\right]^{<\omega}$ de tal forma que vale o item (b).

Para cada $\eta \in 2^{\mathfrak{c}}, \beta \in \alpha$, faça $x_{\eta, \beta}=\left\{\phi_{F}(\{\eta\} \times\{\beta\}): F \in\left[2^{\mathfrak{c}} \times \alpha\right]^{<\omega} \backslash \emptyset\right\} \cup$ $\cup\left\{\psi_{A}(\{\eta\} \times\{\beta\}): A \subseteq \omega \times \alpha\right\}, \operatorname{com} \phi_{F}:\left[2^{\mathfrak{c}} \times \alpha\right]^{<\omega} \longmapsto\{0,1\}$ homomorfismo definido no lema anterior tal que $\phi_{F}(F)=1$. Considere $\left\{x_{\xi, \beta}: \xi<2^{\mathfrak{c}}, \beta \in \alpha\right\}$ enumeração do conjunto formado pelos elementos definidos acima. Dado $\left\{x_{g_{\xi, \beta}(n)}: n \in \omega\right\}$, com $\xi \in\left[\omega, 2^{\mathfrak{c}}\right)$ e $\beta \in A_{\xi}$, temos que $x_{g_{\xi, \beta}(n)}=\left\{\phi_{F}\left(g_{\xi, \beta}(n)\right): F \in\left[2^{\mathfrak{c}} \times \alpha\right]^{<\omega} \backslash \emptyset\right\} \cup\left\{\psi_{A}\left(g_{\xi, \beta}(n)\right): A \subseteq \omega\right\}$, $\forall n \in \omega$. Daí, pela construção dos homomorfismos $\phi_{F}$ e $\psi_{A}$ temos que $x_{\xi, \beta}$ é o $p_{\xi}$-limite de $\left\{x_{g_{\xi, \beta}(n)}: n \in \omega\right\}, \forall \beta \in A_{\xi}$. Se $\lambda \notin A_{\xi}$, temos que $g_{\xi, \lambda}(n)=\triangle\left(\left\{g_{\xi, \eta}(n): \eta \in A_{\xi, \lambda}\right\}\right) \triangle F_{\lambda}$, $\forall n \in \Omega$ elemento de $p_{\xi}$, com $F_{\lambda} \in\left[2^{\mathfrak{c}} \times \alpha\right]^{<\omega}$ e $A_{\xi, \lambda} \subseteq A_{\xi}$. Como $\phi_{F}$ é homomorfismo, $\forall F \in\left[2^{\mathfrak{c}} \times \alpha\right]^{<\omega} \operatorname{temos} \phi_{F}\left(g_{\xi, \lambda}(n)\right)=\sum\left(\left\{\phi_{F}\left(g_{\xi, \eta}(n)\right): \eta \in A_{\xi, \lambda}\right\}\right)+\phi_{F}\left(F_{\lambda}\right), \forall n \in \Omega$. Vale uma expressão semelhante também para todo homomorfismo $\psi_{A}$, com $A \subseteq \omega \times \alpha$. Assim, $x_{g_{\xi, \lambda}(n)}=\sum\left(\left\{x_{g_{\xi, \eta}(n)}: \eta \in A_{\xi, \lambda}\right\}\right)+x^{\prime}, \forall n \in \Omega$. Seja $\Omega_{\eta}=\left\{n \in \omega: x_{g_{\xi, \eta}(n)}=x_{\xi, \eta}\right\} \in$ $p_{\xi}, \forall \eta \in A_{\xi, \lambda}$. Como $A_{\xi, \lambda}$ é finito, temos que $\left\{n \in \omega: x_{g_{\xi, \lambda}(n)}=\sum\left(\left\{x_{\xi, \eta}: \eta \in A_{\xi, \lambda}\right\}\right)+x^{\prime}\right\}$ é elemento de $p_{\xi}$, pois contém $\bigcap\left\{\Omega_{\eta}: \eta \in A_{\xi, \lambda}\right\} \cap \Omega$. Logo, $\sum\left(\left\{x_{\xi, \eta}: \eta \in A_{\xi, \lambda}\right\}\right)+x^{\prime}$ é $\mathrm{p}_{\xi^{-}}$ limite para a sequência $\left\{x_{g_{\xi, \lambda}(n)}: n \in \omega\right\}, \lambda \notin A_{\xi}$. Além disso, como $g_{\xi, \lambda}(n) \subseteq \bigcup_{\beta<\gamma}^{n \in \omega} g_{\xi, \beta}(n)$ ,$g_{\xi, \eta}(n) \subseteq \bigcup_{\beta<\gamma}^{n \in \omega} g_{\xi, \beta}(n) \forall \eta \in A_{\xi, \lambda}$ e $F_{\lambda}=\triangle\left(\left\{g_{\xi, \eta}(n): \eta \in A_{\xi, \lambda}\right\}\right) \triangle g_{\xi, \lambda}(n)$ teremos que $F_{\lambda} \subseteq \bigcup_{\beta<\gamma}^{n \in \omega} g_{\xi, \beta}(n)$. Daí, $x^{\prime}=\left\{\phi_{F}\left(F_{\lambda}\right): F \in\left[2^{\mathfrak{c}} \times \alpha\right]^{<\omega} \backslash \emptyset\right\} \cup\left\{\psi_{A}\left(F_{\lambda}\right): A \subseteq \omega \times \alpha\right\}$ pode 
ser tomado como combinação linear de elementos do conjunto $\left\{x_{\eta, \theta}:(\eta, \theta) \in \bigcup_{\beta<\gamma}^{n \in \omega} g_{\xi, \beta}(n)\right\}$. Logo, $\sum\left(\left\{x_{\xi, \eta}: \eta \in A_{\xi, \lambda}\right\}\right)+x^{\prime}$ está em $\left\langle\left\{x_{\eta, \theta}:(\eta, \theta) \in \bigcup_{\beta<\gamma}^{n \in \omega} g_{\xi, \beta}(n) \cup\{\xi\} \times A_{\xi}\right\}\right\rangle$ e vale (I-a).

O item (II) é obtido da construção dos homomorfismos $\psi_{A}$.

Para o item (III), se $p, q \in \omega^{*}$ são distintos, existem $B_{p} \in p$ e $B_{q} \in q$ tais que $B_{p} \cap B_{q}=\emptyset$ : se $p \neq q$, então existe, digamos, $B_{p} \in p \backslash q$. Daí, como $q$ é ultrafiltro, temos que $\omega \backslash B_{p} \in q$ e $\left(\omega \backslash B_{p}\right) \cap B_{p}=\emptyset$. Faça então $B_{q}=\omega \backslash B_{p}$. Pelo item (II), existe $(\eta, \beta) \in 2^{\mathfrak{c}} \times \alpha$ tal que $\left\{(n, \gamma) \in \omega \times \alpha: x_{n, \gamma}(\eta, \beta)=1\right\}=B_{p} \times\left\{\beta_{1}\right\}$. Temos então que $x_{n, \gamma}(\eta, \beta)=1$, $\forall(n, \gamma) \in B_{p} \times\left\{\beta_{1}\right\}$, e $x_{n, \gamma}(\eta, \beta)=0 \forall(n, \gamma) \in B_{p} \times\left\{\gamma_{1}\right\} \cup B_{q} \times\left\{\beta_{2}\right\} \cup B_{q} \times\left\{\gamma_{2}\right\}$, pois $\left(B_{p} \times\left\{\beta_{1}\right\}\right) \cap\left(B_{p} \times\left\{\gamma_{1}\right\} \cup B_{q} \times\left\{\beta_{2}\right\} \cup B_{q} \times\left\{\gamma_{2}\right\}\right)=\emptyset$. Portanto, o $p$-limite da sequência $\left\{x_{n, \beta_{1}}(\eta, \beta)+x_{n, \gamma_{1}}(\eta, \beta): n \in \omega\right\}$ é 1 e o $q$-limite de $\left\{x_{n, \beta_{2}}(\eta, \beta)+x_{n, \gamma_{2}}(\eta, \beta): n \in \omega\right\}$ é 0 . $\operatorname{Logo}, p-\lim \left\{x_{\left(n, \beta_{1}\right)}+x_{\left(n, \gamma_{1}\right)}: n \in \omega\right\} \neq q-\lim \left\{x_{\left(n, \beta_{2}\right)}+x_{\left(n, \gamma_{2}\right)}: n \in \omega\right\}$. Se $\beta, \gamma \in \alpha$, com $\beta \neq \gamma$, então pelo item (II), existe $(\eta, \mu) \in 2^{\mathfrak{c}} \times \alpha$ tal que $\left\{(n, \xi) \in \omega \times \alpha: x_{n, \xi}(\eta, \mu)=1\right\}=$ $B_{p} \times\{\beta\}$. Como $B_{p} \times\{\beta\} \cap B_{p} \times\{\gamma\}=\emptyset$, temos que $x_{n, \xi}(\eta, \mu)=0, \forall(n, \xi) \in B_{p} \times\{\gamma\}$. Daí, $p-\lim \left\{x_{n, \beta}: n \in \omega\right\} \neq p-\lim \left\{x_{n, \gamma}: n \in \omega\right\}$.

\section{2 $H^{\gamma}$ é enumeravelmente compacto para todo $\gamma<2^{\mathfrak{c}}$, mas $H^{2^{\mathfrak{c}}}$ não é.}

Lema 5.2.1: Seja $\mathcal{F}=\left\{g_{\xi, \beta}: \omega \leq \xi<2^{\mathfrak{c}} \wedge \beta<\gamma<2^{\mathfrak{c}}\right\}$ uma enumeração de todas as funções injetoras em $\left(\left[2^{\mathfrak{c}} \times 2^{\mathfrak{c}}\right]^{<\omega}\right)^{\omega}$ tais que

(a) $\bigcup_{n \in \omega}^{\beta<\gamma} g_{\xi, \beta}(n) \subseteq \xi \times 2^{\mathfrak{c}}$, para todo ordinal infinito $\xi<2^{\mathfrak{c}}$, com $\gamma<2^{\mathfrak{c}}$;

(b) cada $g_{\xi}$ injetora em $\left(\left[2^{\mathfrak{c}} \times 2^{\mathfrak{c}}\right]^{<\omega}\right)^{\omega}$ aparece $2^{\mathfrak{c}}$ vezes na enumeração (Aqui denotamos $g_{\xi}$ por $\left.\left\{g_{\xi, \beta}: \beta \leq \gamma\right\}\right)$ 
(c) existe uma família $\left\{A_{\xi}: \omega \leq \xi<2^{\mathfrak{c}}\right\}$ de subconjuntos de $2^{\mathfrak{c}}$, com $\left|A_{\xi}\right| \leq \gamma<2^{\mathfrak{c}}$, tal que dado $\xi \in 2^{\mathfrak{c}} \backslash \omega,\left\{\left[g_{\xi, \beta}\right]_{p_{\xi}}: \beta \in A_{\xi}\right\} \cup\left\{[\vec{\mu}]_{p_{\xi}}: \mu \in 2^{\mathfrak{c}}\right\}$ é linearmente independente.

Além disso, sejam $\left\{x_{\xi, \beta}: \xi<2^{\mathfrak{c}}, \beta \in 2^{\mathfrak{c}}\right\}$ família que satisfaz as condições (I)-(III) no Lema 5.1.2 para a família $\mathcal{F}$. Seja $\omega^{*}=\left\{q_{\eta}: \eta<2^{\mathfrak{c}}\right\}$ enumeração de todos os ultrafiltros livres. Então, existem famílias $\left\{\mu_{\xi}: \xi<2^{\mathfrak{c}}\right\},\left\{\beta_{\xi}: \xi<2^{\mathfrak{c}}\right\},\left\{I_{\xi}: \xi<2^{\mathfrak{c}}\right\}$ e $\left\{S_{\xi}: \xi<2^{\mathfrak{c}}\right\}$ que satisfazem as seguintes condições:

(i) $I_{\xi}=\omega \times \omega \cup\left\{\left\{\mu_{\eta}\right\} \times A_{\mu_{\eta}}: \eta<\xi\right\} \cup\left\{\{n\} \times\left\{\beta_{\eta}\right\}: n \in \omega \wedge \eta<\xi\right\}, \forall \xi<2^{\mathfrak{c}} \mathrm{e}$ $\mu_{\eta}, \beta_{\eta} \in\left[\omega, 2^{\mathfrak{c}}\right)$ para cada $\eta<\xi$

(ii) se $\eta<\xi<2^{\mathfrak{c}}$ então $S_{\eta} \subseteq S_{\xi}$, com $S_{\xi} \subseteq 2^{\mathfrak{c}} \times 2^{\mathfrak{c}}, \forall \xi<2^{\mathfrak{c}}$;

(iii) $I_{\xi} \cap S_{\xi}=\emptyset$ para cada $\xi<2^{\mathfrak{c}}$;

(iv) $g_{\mu_{\xi}}=g_{\delta_{\xi}}$, onde $\delta_{\xi}$ é o menor ordinal $\delta$ em $\left[\omega, 2^{\mathfrak{c}}\right)$ para o qual $\bigcup_{n \in \omega}^{\beta<\gamma} \operatorname{supp}\left(g_{\delta, \beta}(n)\right) \subseteq I_{\xi}$ e $g_{\delta} \neq g_{\mu_{\eta}}, \forall \eta<\xi$

(v) não existe $q_{\xi}$-limite para $\left\{x_{n, \beta_{\xi}}: n \in \omega\right\}$ ou $q_{\xi}-\lim \left\{x_{n, \beta_{\xi}}: n \in \omega\right\} \notin$ $\notin\left\langle\left\{x_{\eta, \theta}:(\eta, \theta) \in 2^{\mathfrak{c}} \times 2^{\mathfrak{c}} \backslash S_{\xi}\right\}\right\rangle$ para todo $\xi<2^{\mathfrak{c}}$

(vi) $S_{\xi}$ tem cardinalidade menor ou igual a $|\xi|+|\omega|$ para cada $\xi<2^{\mathfrak{c}}$.

Demonstração: Durante a construção por indução, denote por $H_{\xi}$ o grupo gerado por $\left\{x_{\eta, \beta}:(\eta, \beta) \in I_{\xi}\right\}$.

Caso 1. Estágio 0. Defina $I_{0}=\omega \times \omega$.

Definiremos agora $S_{0}$. Seja $q_{0} \in \omega^{*}$. Do fato de que $\left|H_{0}\right|=\left|\left[I_{0}\right]^{<\omega}\right|<2^{\mathfrak{c}}$ temos que existe $\beta_{0} \in\left[\omega, 2^{\mathfrak{c}}\right)$, tal que $q_{0}-\lim x_{n, \beta_{0}}=x_{q_{0}} \notin H_{0}$. Neste caso, teremos $\operatorname{supp} x_{q_{0}} \backslash I_{0} \neq \emptyset$ e podemos fixar $\theta_{0} \in \operatorname{supp}\left(x_{q_{0}}\right) \backslash I_{0}$. Faremos $S_{0}=\left\{\theta_{0}\right\}$.

Seja agora $\xi_{0}$ o menor ordinal tal que $\bigcup_{n \in \omega}^{\beta<\gamma} \operatorname{supp}\left(g_{\xi_{0}, \beta}(n)\right) \subseteq I_{0}$. Como cada $g$ injetora em $\left(\left[2^{\mathfrak{c}} \times 2^{\mathfrak{c}}\right]^{<\omega}\right)^{\omega}$ aparece $2^{\mathfrak{c}}$ vezes na enumeração, existe $\mu_{0} \in\left[\omega, 2^{\mathfrak{c}}\right)$ tal que $\left[\left\{\mu_{0}\right\} \times A_{\mu_{0}}\right] \cap\left[I_{0} \cup S_{0}\right]=\emptyset$ e $g_{\mu_{0}}=g_{\xi_{0}}$. Dessa forma obtemos $\mu_{0}$. 
Caso 2. Suponha por indução que $\left\{\mu_{\xi}: \xi<\lambda+1\right\},\left\{I_{\xi}: \xi<\lambda+1\right\}$, e $\left\{S_{\xi}: \xi<\lambda+1\right\}$ estejam definidos. Como $\mu_{\xi}, \beta_{\xi}$ já estão definidos para todo $\xi \leq \lambda$, temos que $I_{\lambda+1}=\omega \times \omega \cup\left\{\left\{\mu_{\eta}\right\} \times A_{\mu_{\eta}}: \eta<\lambda+1\right\} \cup\left\{\{n\} \times\left\{\beta_{\eta}\right\}: n \in \omega \wedge \eta<\lambda+1\right\}$ satisfaz a condição (i).

Seja $\eta=\lambda+1$. Definiremos agora $S_{\eta}$. Seja $q_{\eta} \in \omega^{*}$. Afirmação: podemos tomar uma sequência $\left\{x_{n, \beta_{\eta}}: n \in \omega\right\}$ tal que

(a) $\left\{x_{n, \beta_{\eta}}: n \in \omega\right\} \cap\left(H_{\eta} \cup S_{\lambda}\right)=\emptyset \mathrm{e}$

(b) o $q_{\eta}$-limite de $\left\{x_{n, \beta_{\eta}}: n \in \omega\right\}$ não existe ou não está em $H_{\eta}$.

Do fato de que $\left|H_{\eta}\right|=\left|\left[I_{\eta}\right]^{<\omega}\right|<2^{\mathfrak{c}}$ e $\left|S_{\lambda}\right| \leq \eta$ temos que a cardinalidade do conjunto formado pelos $\beta \in\left[\omega, 2^{\mathfrak{c}}\right)$ tais que $\left\{x_{n, \beta}: n \in \omega\right\} \cap\left(H_{\eta} \cup S_{\lambda}\right)=\emptyset$ é igual a $2^{\mathfrak{c}}$. Em particular, vale (a). Suponha que $\exists q_{\eta}$-limite para todo $\beta$ do conjunto acima. Pelo item (III) do lema 5.1 .2 , se $\beta \neq \gamma$ então $q_{\eta}-\lim \left\{x_{n, \beta}: n \in \omega\right\} \neq q_{\eta}-\lim \left\{x_{n, \gamma}: n \in \omega\right\}$ de forma que a cardinalidade do conjunto de $q_{\eta}$-limites é $2^{\mathfrak{c}}$ enquanto que $\left|H_{\eta}\right|=\left|\left[I_{\eta}\right]^{<\omega}\right|<2^{\mathfrak{c}}$. Logo podemos tomar $\beta_{\eta}<2^{\mathfrak{c}}$ tal que $q_{\eta}-\lim x_{n, \beta_{\eta}}=x_{q_{\eta}} \notin H_{\eta}$. Segue-se a afirmação.

Neste caso, teremos $\operatorname{supp}\left(x_{q_{\eta}}\right) \backslash I_{\eta} \neq \emptyset$ e podemos fixar $\theta_{q_{\eta}} \in \operatorname{supp}\left(x_{q_{\eta}}\right) \backslash I_{\eta}$. Faremos $S_{\eta}=S_{\lambda} \cup\left\{\theta_{q_{\eta}}\right\}$. Vê-se facilmente que os itens (ii), (v) e (vi) são verificados a partir desta definição.

Seja agora $\delta_{\eta}$ o menor ordinal tal que $\bigcup_{n \in \omega}^{\beta<\alpha} \operatorname{supp}\left(g_{\delta_{\eta}, \beta}(n)\right) \subseteq I_{\eta}$ e $g_{\delta_{\eta}} \neq g_{\mu_{\beta}}, \forall \beta<\eta$. Como cada $g$ injetora em $\left(\left[2^{\mathfrak{c}} \times 2^{\mathfrak{c}}\right]^{<\omega}\right)^{\omega}$ aparece $2^{\mathfrak{c}}$ vezes na enumeração, existe $\mu_{\eta} \in\left[\omega, 2^{\mathfrak{c}}\right)$ tal que $\left[\left\{\mu_{\eta}\right\} \times A_{\mu_{\eta}}\right] \cap\left[I_{\eta} \cup S_{\eta}\right]=\emptyset$ e $g_{\mu_{\eta}}=g_{\delta_{\eta}}$. Isto é possível, pois $\left|I_{\eta} \cup S_{\eta}\right|<2^{\mathfrak{c}}$. Dessa forma obtemos $\mu_{\eta}$ e como no parágrafo anterior já havíamos determinado $\beta_{\eta}$, valem os itens (iii) e (iv). 
Caso 3. Seja $\xi$ ordinal limite menor que $2^{\mathfrak{c}}$. Temos que $I_{\xi}=\bigcup_{\eta<\xi} I_{\eta}=\omega \times \omega \cup$ $\cup\left\{\left\{\mu_{\eta}\right\} \times A_{\mu_{\eta}}: \eta<\xi\right\} \cup\left\{\{n\} \times\left\{\beta_{\eta}\right\}: n \in \omega \wedge \eta<\xi\right\}$ como em (i). Defina $S=\bigcup_{\eta<\xi} S_{\eta}$. Definiremos agora $S_{\xi}$. Como no caso anterior, podemos tomar uma sequência $\left\{x_{n, \beta_{\xi}}: n \in \omega\right\}$ tal que $\left\{x_{n, \beta_{\xi}}: n \in \omega\right\} \cap\left(H_{\xi} \cup S\right)=\emptyset$ e o $q_{\xi}$-limite de $\left\{x_{n, \beta_{\xi}}: n \in \omega\right\}$ não existe ou não está em $H_{\xi}$. Caso o $q_{\xi}$-limite de $\left\{x_{n, \beta}: n \in \omega\right\}$ exista $\forall \beta$, tome $\beta_{\xi}$ tal que $x_{q_{\xi}}=q_{\xi}-\lim \left\{x_{n, \beta_{\xi}}: n \in \omega\right\} \notin H_{\xi}$. Teremos $\operatorname{supp}\left(x_{q_{\xi}}\right) \backslash I_{\xi} \neq \emptyset$ e podemos fixar $\theta_{q_{\xi}} \in \operatorname{supp}\left(x_{q_{\xi}}\right) \backslash I_{\xi}$. Poremos $S_{\xi}=S \cup\left\{\theta_{q_{\xi}}\right\}$. Vê-se facilmente que os itens (ii), (iii), (v) e (vi) são verificados a partir desta definição.

Seja $\delta_{\xi}$ o menor ordinal $\delta$ em $\left[\omega, 2^{\mathfrak{c}}\right)$ para o qual $\bigcup_{n \in \omega}^{\beta<\gamma} \operatorname{supp}\left(g_{\delta, \beta}(n)\right) \subseteq I_{\xi}$ e $g_{\delta} \neq g_{\mu_{\eta}}$, $\forall \eta<\xi$, e escolha $\mu_{\xi} \in\left[\omega, 2^{\mathfrak{c}}\right)$ de tal forma que $g_{\mu_{\xi}}=g_{\delta_{\xi}}$ e $\left[\left\{\mu_{\xi}\right\} \times A_{\mu_{\xi}}\right] \cap\left[I_{\xi} \cup S_{\xi}\right]=\emptyset$. Novamente isto é possível, pois $I_{\xi} \cup S_{\xi}$ tem cardinalidade menor que $2^{\mathfrak{c}}$. Assim, a condição (iv) é também satisfeita.

Exemplo 5.2.1: Seja $I=\bigcup_{\xi<2^{c}} I_{\xi}$ e $H=\left\langle\left\{x_{\xi, \beta}:(\xi, \beta) \in I\right\}\right\rangle$. Então $H^{\gamma}$ é enumeravelmente compacto para todo $\gamma<2^{\mathfrak{c}}$, mas $H^{2^{\mathfrak{c}}}$ não é enumeravelmente compacto.

De fato, seja $\gamma<2^{\mathfrak{c}}$ e considere $\left\{t_{n, \lambda}: n \in \omega \wedge \lambda \in \gamma+1\right\}$ sequência 1-1 em $H^{\gamma}$. Então existe $h_{n, \lambda} \in \mathbb{Z}_{2}^{(I)}$ tal que $t_{n, \lambda}=x_{h_{n, \lambda}}$ para todo $n \in \omega$. Seja $\eta<2^{\mathfrak{c}}$ tal que $\bigcup_{n \in \omega}^{\lambda \in \gamma+1} \operatorname{supp}\left(h_{n, \lambda}\right) \subseteq I_{\eta}$ e seja $\theta$ o menor ordinal tal que $g_{\theta, \lambda}(n)=\operatorname{supp}\left(h_{n, \lambda}\right) \forall n \in \omega \mathrm{e}$ $\lambda \in \gamma+1$. Daí, existe $\mu_{\xi}<2^{\mathfrak{c}}$ tal que $g_{\mu_{\xi}}=g_{\theta}$ e a sequência $\left\{t_{n, \lambda}: n \in \omega \wedge \lambda \in \gamma+1\right\}$ pode ser vista como $\left\{x_{g_{\mu_{\xi}, \lambda}(n)}: n \in \omega \wedge \lambda \in \gamma+1\right\}$. Agora, para todo $\lambda \leq \gamma$, se $\lambda \in A_{\mu_{\xi}}$, temos pelo item (I) do lema anterior que $x_{\mu_{\xi}, \lambda}$ é o $p_{\mu_{\xi}}$ limite de $\left\{x_{g_{\mu_{\xi}, \lambda}(n)}: n \in \omega\right\}$. Se $\lambda \notin A_{\mu_{\xi}}$, temos do item (I-a) do lema anterior que a sequência $\left\{x_{g_{\mu_{\xi}, \lambda}(n)}: n \in \omega\right\}$ possui $\mathrm{p}_{\mu_{\xi}}$-limite em $\left.\left\langle\left\{x_{\eta, \theta}:(\eta, \theta) \in \bigcup_{n \in \omega}^{\beta \in \gamma} g_{\mu_{\xi}, \beta}(n)\right\} \cup\left\{\mu_{\xi}\right\} \times A_{\mu_{\xi}}\right\}\right\rangle \subseteq\left\langle\left\{x_{\xi, \beta}:(\xi, \beta) \in I_{\xi+1}\right\}\right\rangle$. Como $\left\langle\left\{x_{\xi, \beta}:(\xi, \beta) \in I_{\xi+1}\right\}\right\rangle \subseteq H$ para todo $\lambda \in \gamma+1$, temos que $H^{\gamma}$ é enumeravelmente compacto. 
Por outro lado, $H^{2^{c}}$ não é enumeravelmente compacto. De fato, a sequência $\left\{\left(x_{n, \beta_{\xi}}\right)_{\xi \in 2^{\mathfrak{c}}}: n \in \omega\right\}$ não possui q-limite em $H^{2^{\mathfrak{c}}}$ para todo $q \in \omega^{*}$, pois dado $q \in \omega^{*}$ temos que $q=q_{\eta}$ para algum $\eta \in 2^{\mathfrak{c}}$. Daí, pelo item $(\mathrm{v})$ a sequência $\left\{x_{n, \beta_{\eta}}: n \in \omega\right\}$ não possui $q_{\eta}$-limite ou $q_{\eta}-\lim \left\{x_{n, \beta_{\eta}}: n \in \omega\right\} \notin\left\langle\left\{x_{\eta, \theta}:(\eta, \theta) \in 2^{\mathfrak{c}} \times 2^{\mathfrak{c}} \backslash S_{\eta}\right\}\right\rangle$. Do item (iii), temos que $I \cap S_{\eta}=\emptyset$ de forma que $H \subseteq\left\langle\left\{x_{\eta, \theta}:(\eta, \theta) \in 2^{\mathfrak{c}} \times 2^{\mathfrak{c}} \backslash S_{\eta}\right\}\right\rangle$. Assim, tanto no primeiro caso como no segundo vemos que a sequência $\left\{x_{n, \beta_{\eta}}: n \in \omega\right\}$ não possui $q_{\eta}$-limite em $H$. Logo, $\left\{\left(x_{n, \beta_{\xi}}\right)_{\xi \in 2^{\mathfrak{c}}}: n \in \omega\right\}$ não possui ponto de acumulação.

Segue-se então que $H^{2^{\mathfrak{c}}}$ não é enumeravelmente compacto.

\section{3 $H^{\gamma}$ é enumeravelmente compacto para todo $\gamma<\alpha$, mas $H^{\alpha}$ não é $\left(\omega \leq \alpha<2^{\mathfrak{c}}\right)$.}

Lema 5.3.1: Seja $\mathcal{F}=\left\{g_{\xi, \beta}: \omega \leq \xi<2^{\mathfrak{c}} \wedge \beta<\gamma<\alpha\right\}$ uma enumeração de todas as funções injetoras em $\left(\left[2^{\mathfrak{c}} \times \alpha\right]^{<\omega}\right)^{\omega}$, $\operatorname{com} \alpha<2^{\mathfrak{c}}$, tal que

(a) $\bigcup_{n \in \omega}^{\beta<\gamma} g_{\xi, \beta}(n) \subseteq \xi \times \alpha$, para todo ordinal infinito $\xi<2^{\mathfrak{c}}$, e

(b) cada $g_{\xi}$ injetora em $\left(\left[2^{\mathfrak{c}} \times \alpha\right]^{<\omega}\right)^{\omega}$ aparece $2^{\mathfrak{c}}$ vezes na enumeração (Aqui denotamos $g_{\xi}$ por $\left.\left\{g_{\xi, \beta}: \beta<\gamma\right\}\right)$;

(c) existe uma família $\left\{A_{\xi}: \omega \leq \xi<2^{\mathfrak{c}}\right\}$ de subconjuntos de $\alpha$, com $\left|A_{\xi}\right| \leq \gamma<\alpha$, tal que dado $\xi \in 2^{\mathfrak{c}} \backslash \omega,\left\{\left[g_{\xi, \beta}\right]_{p_{\xi}}: \beta \in A_{\xi}\right\} \cup\left\{[\vec{\mu}]_{p_{\xi}}: \mu \in 2^{\mathfrak{c}}\right\}$ é linearmente independente.

$\operatorname{Sejam~}\left\{x_{\xi, \beta}: \xi<2^{\mathfrak{c}}, \beta \in \alpha\right\}$ e $\left\{p_{\xi}: \omega \leq \xi<2^{\mathfrak{c}}\right\}$ famílias que satisfazem as condições (I)-(III) no lema 5.1.2 para a família $\mathcal{F}$. Então existem famílias $\left\{\mu_{\xi}: \xi<2^{\mathfrak{c}}\right\},\left\{I_{\xi}: \xi<2^{\mathfrak{c}}\right\}$, $\left\{S_{\xi}: \xi<2^{\mathfrak{c}}\right\}$ e $\left\{P_{\xi}: \xi<2^{\mathfrak{c}}\right\}$ que satisfazem as seguintes condições: 
(i) $I_{\xi}=\omega \times \alpha \cup\left\{\left\{\mu_{\eta}\right\} \times A_{\mu_{\eta}}: \eta<\xi\right\}, \forall \xi<2^{\mathfrak{c}}$ e $\mu_{\eta}<2^{\mathfrak{c}}$ para cada $\eta<\xi$;

(ii) $S_{\xi} \subseteq 2^{\mathfrak{c}} \times \alpha, \forall \xi<2^{\mathfrak{c}}$;

(iii) $\left(I_{\xi} \cup\left\{\mu_{\xi}\right\} \times A_{\mu_{\xi}}\right) \cap S_{\xi}=\emptyset$ para cada $\xi<2^{\text {c }}$;

(iv) $g_{\mu_{\xi}}=g_{\delta_{\xi}}$, onde $\delta_{\xi}$ é o menor ordinal $\delta$ em $\left[\omega, 2^{\mathfrak{c}}\right)$ para o qual $\bigcup_{\beta<\gamma}^{n \in \omega} \operatorname{supp}\left(g_{\delta, \beta}(n)\right) \subseteq I_{\xi}$ e $g_{\delta} \neq g_{\mu_{\eta}}, \forall \eta<\xi$

(v) $P_{\xi}=\left\{p: p-\lim \left\{x_{n, \beta}: n \in \omega\right\} \in\left\langle x_{\eta, \mu}:(\eta, \mu) \in 2^{\mathfrak{c}} \times \alpha\right\rangle, \forall \beta<\alpha\right.$ e $\exists \beta_{1}, \beta_{2}, \beta_{1} \neq \beta_{2}$, tais que $\left.p-\lim \left\{x_{n, \beta_{1}}+x_{n, \beta_{2}}: n \in \omega\right\} \in H_{\xi}\right\}, \forall \xi<2^{\text {c }}$;

(vi) se $p \in P_{\xi}$ então existe $\beta<\alpha$ tal que $p-\lim \left\{x_{n, \beta}: n \in \omega\right\} \notin$ $\notin\left\langle\left\{x_{\eta, \theta}:(\eta, \theta) \in 2^{\mathfrak{c}} \times \alpha \backslash S_{\xi}\right\}\right\rangle$;

(vii) $P_{\xi}$ tem cardinalidade menor ou igual que $|\alpha|+|\xi|$ para cada $\xi<2^{\mathfrak{c}}$;

(viii) $S_{\xi}$ tem cardinalidade menor ou igual que $|\alpha|+|\xi|$ para cada $\xi<2^{\text {c }}$;

(ix) se $\eta<\xi<2^{\text {c }}$, então $P_{\eta} \subseteq P_{\xi}$ e se $\xi$ é ordinal limite, então $P_{\xi}=\bigcup_{\eta<\xi} P_{\eta}$;

(x) se $\eta<\xi<2^{\mathfrak{c}}$, então $S_{\eta} \subseteq S_{\xi}$ e se $\xi$ é ordinal limite, então $S_{\xi}=\bigcup_{\eta<\xi} S_{\eta}$.

Demonstração: Durante a construção por indução, denote por $H_{\xi}$ o grupo gerado por $\left\{x_{\eta, \beta}:(\eta, \beta) \in I_{\xi}\right\}$.

Caso 1. Estágio 0. Defina $I_{0}=\omega \times \alpha$. Temos que $\left\{p: p-\lim \left\{x_{n, \beta}: n \in \omega\right\} \in\right.$ $\left\langle x_{\eta, \mu}:(\eta, \mu) \in 2^{\mathfrak{c}} \times \alpha\right\rangle, \forall \beta<\alpha$ e $\exists \beta_{1}, \beta_{2}$ tais que $\left.p-\lim \left\{x_{n, \beta_{1}}+x_{n, \beta_{2}}: n \in \omega\right\} \in H_{0}\right\}=$ $=\emptyset$. De fato, se $x_{F} \in H_{0} \operatorname{com} F \in[\omega \times \alpha]^{<\omega} \backslash \emptyset$, então pelo item (II) do lema 5.1.2, existe $(\eta, \beta) \in 2^{\mathfrak{c}} \times \alpha$ tal que $\left\{(n, \gamma) \in \omega \times \alpha: x_{n, \gamma}(\eta, \beta)=1\right\}=\{(m, \mu)\}$, com $(m, \mu) \in F$. Daí, temos que $x_{F}(\eta, \beta)=1$ e $p-\lim \left\{x_{n, \beta_{1}}(\eta, \beta)+x_{n, \beta_{2}}(\eta, \beta): n \in \omega\right\}=0$, pois $\pi_{1}(F)$ é finito e, portanto, $\omega \backslash \pi_{1}(F) \in p$. Logo, $P_{0}=\emptyset$ e as condições (vi), (vii) e (ix) são trivialmente satisfeitas. Defina $S_{0}=\emptyset$ e faça $\mu_{0}=\omega$. As condições (ii), (iii), (iv), (viii) e (x) são claramente satisfeitas.

Caso 2. Suponha por indução que $\left\{\mu_{\xi}: \xi<\lambda+1\right\},\left\{I_{\xi}: \xi<\lambda+1\right\},\left\{S_{\xi}: \xi<\lambda+1\right\}$ 
e $\left\{P_{\xi}: \xi<\lambda+1\right\}$ estejam definidos. Como $\mu_{\xi}$ já está definido para todo $\xi \leq \lambda$, temos que $I_{\lambda+1}=\omega \times \alpha \cup\left\{\left\{\mu_{\eta}\right\} \times A_{\mu_{\eta}}: \eta<\lambda+1\right\}$ satisfaz a condição (i).

Esteja $P_{\lambda+1}$ definido como no item (v). Como $H_{\lambda} \subseteq H_{\lambda+1}$ temos que vale o item (ix). Temos também que para cada $p \in P_{\lambda+1}, \exists \beta_{1}, \beta_{2} \in \alpha, \beta_{1} \neq \beta_{2}$, tais que $x_{p}=$ $p-\lim \left\{x_{n, \beta_{1}}+x_{n, \beta_{2}}: n \in \omega\right\} \in H_{\lambda+1}$. Pelo item (III) do lema 5.1.2, se $p \neq q$ em $P_{\lambda+1}$, então $x_{p} \neq x_{q}$. Dessa forma temos que $\left|P_{\lambda+1}\right| \leq\left|H_{\lambda+1}\right|=|\alpha|+|\lambda+1|$ e vale (vii).

Definiremos agora $S_{\lambda+1}$. Seja $p \in P_{\lambda+1} \backslash P_{\lambda}$. Afirmamos que existe $\beta<\alpha$ tal que $p-\lim x_{n, \beta}=x_{p} \notin H_{\lambda+1}$. Caso contrário, teríamos que para todo $\mu<\alpha, p-\lim x_{n, \mu}=$ $x_{F_{\mu}}+x_{O_{\mu}}, \operatorname{com} x_{F_{\mu}} \in H_{\gamma}$ e $O_{\mu} \subseteq A_{\lambda}$. Mas como $\left|A_{\lambda}\right|<\alpha \Longrightarrow\left|\left[A_{\lambda}\right]^{<\omega}\right|<\alpha$, temos que existe $\mu_{1} \neq \mu_{2}$ tal que $O_{\mu_{1}}=O_{\mu_{2}}$. Daí, como $\{0,1\}$ é corpo de característica 2 temos que $x_{F_{\mu_{1}}}+x_{O_{\mu_{1}}}+x_{F_{\mu_{2}}}+x_{O_{\mu_{2}}}=x_{F_{\mu_{1}}}+x_{F_{\mu_{2}}} \in H_{\lambda}$, ou seja, $p-\lim x_{n, \mu_{1}}+p-\lim x_{n, \mu_{2}} \in H_{\lambda}$. Chegamos então à uma contradição, pois $p \in P_{\lambda+1} \backslash P_{\lambda}$. Logo, sempre existe $\beta<\alpha$ tal que $p-\lim x_{n, \beta}=x_{p} \notin H_{\lambda+1}$. Neste caso, teremos supp $x_{p} \backslash I_{\lambda+1} \neq \emptyset$ e podemos fixar $\theta_{p} \in \operatorname{supp}\left(x_{p}\right) \backslash I_{\lambda+1}$. Poremos $S_{\lambda+1}=S_{\lambda} \cup\left\{\theta_{p}: p \in P_{\lambda+1} \backslash P_{\lambda}\right\}$. Vê-se facilmente que os itens (ii), (vi), (viii) e (x) são verificados a partir desta definição.

Seja agora $\delta_{\lambda+1}$ o menor ordinal tal que $\bigcup_{n \in \omega}^{\beta \leq \gamma} g_{\delta_{\lambda+1}, \beta}(n) \subseteq \omega \times \alpha \cup \underset{\beta<\lambda+1}{\bigcup}\left\{\mu_{\beta}\right\} \times$ $A_{\mu_{\beta}}$ e $g_{\delta_{\lambda+1}} \neq g_{\mu_{\beta}}, \forall \beta<\gamma+1$. Como cada $g$ injetora em $\left(\left[2^{\mathfrak{c}} \times \alpha\right]^{<\omega}\right)^{\omega}$ aparece $2^{\mathfrak{c}}$ vezes na enumeração, existe $\mu_{\lambda+1} \in\left[\omega, 2^{\mathfrak{c}}\right)$ tal que $\left[\left\{\mu_{\lambda+1}\right\} \times A_{\mu_{\lambda+1}}\right] \cap[(\omega \times \alpha) \cup$ $\left.\cup\left(\bigcup_{\beta<\lambda+1}\left\{\mu_{\beta}\right\} \times A_{\mu_{\beta}}\right) \cup S_{\lambda+1}\right]=\emptyset$ e $g_{\mu_{\lambda+1}}=g_{\delta_{\lambda+1}}$. Isto é possível, pois fizemos com que $S_{\lambda+1}$ tivesse cardinalidade menor ou igual que $|\alpha|+|\lambda+1|$. Dessa forma obtemos $\mu_{\lambda+1} \mathrm{e}$ valem os itens (iii) e (iv).

Caso 3. Seja $\xi$ ordinal limite. Defina $P_{\xi}=\bigcup_{\eta<\xi} P_{\eta}$ como em (ix), $S_{\xi}=\bigcup_{\eta<\xi} S_{\eta}$ como em (x) e $I_{\xi}=\bigcup_{\eta<\xi} I_{\eta}=\omega \times \alpha \cup\left\{\left\{\mu_{\eta}\right\} \times A_{\mu_{\eta}}: \eta<\xi\right\}$ como em (i). Os itens (ii), (vii) e (viii) são claramente satisfeitos. 
(v) é satisfeito:

Seja $p \in P_{\xi}$. Então existe $\eta<\xi$ tal que $p \in P_{\eta}$. Pelo item (v) para $\eta, \exists \beta_{1}, \beta_{2}, \beta_{1} \neq \beta_{2}$, tais que $p-\lim \left\{x_{n, \beta_{1}}+x_{n, \beta_{2}}: n \in \omega\right\} \in\left\langle\left\{x_{\lambda, \beta}:(\lambda, \beta) \in I_{\eta}\right\}\right\rangle \subseteq\left\langle\left\{x_{\lambda, \beta}:(\lambda, \beta) \in I_{\xi}\right\}\right\rangle$. Logo, $P_{\xi} \subseteq\left\{p: p-\lim \left\{x_{n, \beta}: n \in \omega\right\} \in\left\langle x_{\lambda, \mu}:(\lambda, \mu) \in 2^{\mathfrak{c}} \times \alpha\right\rangle, \forall \beta<\alpha\right.$ e $\exists \beta_{1}, \beta_{2}, \beta_{1} \neq \beta_{2}$, tais que $\left.p-\lim \left\{x_{n, \beta_{1}}+x_{n, \beta_{2}}: n \in \omega\right\} \in H_{\xi}\right\}$. Por outro lado, suponha que $\exists \beta_{1}, \beta_{2}, \beta_{1} \neq \beta_{2}$, tais que $p-\lim \left\{x_{n, \beta_{1}}+x_{n, \beta_{2}}: n \in \omega\right\} \in\left\langle\left\{x_{\lambda, \beta}:(\lambda, \beta) \in I_{\xi}\right\}\right\rangle$. Então existe $\eta<\xi$ tal que $p-\lim \left\{x_{n, \beta_{1}}+x_{n, \beta_{2}}: n \in \omega\right\} \in\left\langle\left\{x_{\lambda, \beta}:(\lambda, \beta) \in I_{\eta}\right\}\right\rangle$. Pelo item (v) para $\eta$ segue-se que $p \in P_{\eta} \subset P_{\xi}$ e vale a inclusão inversa.

(vi) é satisfeito:

Pelo item (ix), se $p \in P_{\xi}$ então existe $\eta<\xi$ tal que $p \in P_{\eta}$. Pelo item (vi) para $\eta$, existe $\beta \in \alpha$ tal que $p-\lim \left\{x_{n, \beta}: n \in \omega\right\} \notin\left\langle\left\{x_{\lambda, \theta}:(\lambda, \theta) \in 2^{\mathfrak{c}} \times \alpha \backslash S_{\eta}\right\}\right\rangle$. Mas como $S_{\eta} \subseteq S_{\xi}$ temos que $p-\lim \left\{x_{n, \beta}: n \in \omega\right\} \notin\left\langle\left\{x_{\lambda, \theta}:(\lambda, \theta) \in 2^{\mathfrak{c}} \times \alpha \backslash S_{\xi}\right\}\right\rangle$. Logo, segue-se que o item (vi) é satisfeito.

Seja $\delta_{\xi}$ como no item (iv) e escolha $\mu_{\xi} \in\left[\omega, 2^{\mathfrak{c}}\right.$ ) de tal forma que $g_{\mu_{\xi}}=g_{\delta_{\xi}}$ e $\left[\left\{\mu_{\xi}\right\} \times A_{\mu_{\xi}}\right] \cap\left[(\alpha \times \omega) \cup\left(\bigcup_{\eta<\xi}\left\{\mu_{\eta}\right\} \times A_{\mu_{\eta}}\right) \cup S_{\xi}\right]=\emptyset$. Novamente isto é possível, pois fizemos com que $S_{\xi}$ tivesse cardinalidade menor ou igual que $|\alpha|+|\xi|$. Assim, as condições (iii) e (iv) são também satisfeitas.

Exemplo 5.3.1: Seja $I=\bigcup_{\xi<2^{c}} I_{\xi}$ e seja $H=\left\langle\left\{x_{\xi, \beta}:(\xi, \beta) \in I\right\}\right\rangle$. Então $H^{\gamma}$ é enumeravelmente compacto para todo $\gamma<\alpha$, mas $H^{\alpha}$ não é enumeravelmente compacto.

Demonstração: De fato, seja $\gamma<\alpha$ e considere $\left\{t_{n, \lambda}: n \in \omega \wedge \lambda \in \gamma+1\right\}$ sequência 1-1 em $H^{\gamma}$. Então existe $h_{n, \lambda} \in \mathbb{Z}_{2}^{(I)}$ tal que $t_{n, \lambda}=x_{h_{n, \lambda}}$ para todo $n \in \omega$. Seja $\eta<2^{\mathfrak{c}}$ tal que $\bigcup_{n \in \omega}^{\lambda \in \gamma+1} \operatorname{supp}\left(h_{n, \lambda}\right) \subseteq I_{\eta}$ e seja $\theta$ o menor ordinal tal que $g_{\theta, \lambda}(n)=\operatorname{supp}\left(h_{n, \lambda}\right) \forall n \in \omega$ e $\lambda \in \gamma+1$. Daí, existe $\mu_{\xi}<2^{\mathfrak{c}}$ tal que $g_{\mu_{\xi}}=g_{\theta}$ e a sequência $\left\{t_{n, \lambda}: n \in \omega \wedge \lambda \in \gamma+1\right\}$ 
pode ser vista como $\left\{x_{g_{\mu_{\xi}, \lambda}(n)}: n \in \omega \wedge \lambda \in \gamma+1\right\}$. Agora, para todo $\lambda \leq \gamma$, se $\lambda \in A_{\mu_{\xi}}$, temos pelo item (I) do lema 5.1 .2 que $x_{\mu_{\xi}, \lambda}$ é o $p_{\mu_{\xi}}$ limite de $\left\{x_{g_{\mu_{\xi}, \lambda}(n)}: n \in \omega\right\}$. Se $\lambda \notin A_{\mu_{\xi}}$, temos do item (I-a) do lema 5.1.2 que a sequência $\left\{x_{g_{\mu_{\xi}, \lambda}(n)}: n \in \omega\right\}$ possui $p_{\mu_{\xi}}$-limite em $\left\langle\left\{x_{\eta, \theta}:(\eta, \theta) \in \bigcup_{\beta<\gamma}^{n \in \omega} g_{\mu_{\xi}, \beta}(n) \cup\left\{\mu_{\xi}\right\} \times A_{\mu_{\xi}}\right\}\right\rangle \subseteq\left\langle\left\{x_{\xi, \beta}:(\xi, \beta) \in I_{\xi+1}\right\}\right\rangle$. Como $\left\langle\left\{x_{\xi, \beta}:(\xi, \beta) \in I_{\xi+1}\right\}\right\rangle \subseteq H$ para todo $\lambda \in \gamma+1$, temos que $H^{\gamma}$ é enumeravelmente compacto.

Por outro lado, $H^{\alpha}$ não é enumeravelmente compacto. De fato, afirmamos que a sequência $\left\{\left(x_{n, \beta}\right)_{\beta \in \alpha}: n \in \omega\right\}$ não possui ponto de acumulação.

Suponha por contradição que a sequência $\left\{\left(x_{n, \beta}\right)_{\beta \in \alpha}: n \in \omega\right\}$ possui ponto de acumulação em $H^{\alpha}$. Então $\forall \beta<\alpha,\left\{x_{n, \beta}: n \in \omega\right\}$ possui ponto de acumulação em $H=\bigcup_{\eta<2^{\mathfrak{c}}} H_{\eta}$ e, portanto, existe $\xi<2^{\mathfrak{c}}$ tal que todos os pontos de acumulação estão em $H_{\xi}$. Seja $p \in \omega^{*}$ tal que $\left(x_{\beta}\right)_{\beta \in \alpha}=p-\lim \left\{\left(x_{n, \beta}\right)_{\beta \in \alpha}: n \in \omega\right\} \in\left(H_{\xi}\right)^{\alpha} \subseteq H^{\alpha}$. Pelo item (v), vemos facilmente que $p \in P_{\xi}$. Daí, pelo item (vi), existe $\beta<\alpha$ tal que $p-\lim \left\{x_{n, \beta}: n \in \omega\right\} \notin\left\langle\left\{x_{\eta, \theta}:(\eta, \theta) \in 2^{\mathfrak{c}} \times \alpha \backslash S_{\xi}\right\}\right\rangle$. Como $H=\left\langle\left\{x_{\xi, \beta}:(\xi, \beta) \in I\right\}\right\rangle \mathrm{e}$ $I \cap S_{\xi}=\emptyset$, temos que $p-\lim \left\{x_{n, \beta}: n \in \omega\right\} \notin H$, o que é contradição.

Segue-se então que $H^{\alpha}$ não é enumeravelmente compacto. 


\section{Capítulo 6}

\section{Considerações finais}

Neste capítulo discutiremos um pouco alguns resultados mais recentes relacionados ao tema da dissertação, muito deles sendo encontrados na presente data ainda sob a forma de preprint.

Recentemente foi contruído um grupo enumeravelmente compacto sem sequências nãotriviais convergentes sem a necessidade da existência de um ultrafiltro seletivo como hipótese. Este resultado pode ser encontrado em um artigo publicado on-line no Topology and its Applications por Tomita e Szeptycki ${ }^{1}$. Assim, os exemplos do capítulo 3 obtidos a partir de um grupo de van Douwen podem ser obtidos de forma independente da existência de ultrafiltros seletivos.

Por outro lado, foi demonstrado recentemente em um preprint por Tomita e Sanchis que a existência de um ultrafiltro seletivo é suficiente para construir um exemplo de um grupo enumeravelmente compacto tal que dado $\alpha \leq \omega_{1}$ temos que $G^{\gamma}$ é enumeravelmente compacto para todo $\gamma<\alpha$, mas $G^{\alpha}$ não é enumeravelmente compacto.

O primeiro exemplo de grupo abeliano livre enumeravelmente compacto é de Tkachenko

\footnotetext{
${ }^{1}$ P.J. Szeptycki, A.H. Tomita, HFD groups in the Solovay model, Topology and its Applications (2009)
} 
(Izvestia VUZ, 1990) sob CH, e o mais atual é de Madariaga Garcia e Tomita (Topology Appl.,2007) sob a existencia de $\mathfrak{c}$ ultrafiltros seletivos. Recentemente em um preprint Tomita e Boero usando $\mathfrak{p}=\mathfrak{c}$ associaram uma topologia a um grupo abeliano livre de cardinalidade continuum cujo quadrado é enumeravelmente compacto. Em um artigo de 1998 (Comment. Math. Univ. Carol., 1998), Tomita mostra que uma topologia num grupo abeliano livre não tem $\omega$-potencia enumeravelmente compacta. Uma continuação natural que ainda se encontra por fazer é o estudo da preservação da compacidade enumerável em produtos finitos de grupos abelianos livres que admitem uma topologia enumeravelmente compacta. Dikranjan e Tkachenko (Forum Math.,2003) classificaram usando MA todos os grupos abelianos de cardinalidade $\mathfrak{c}$ que admitem uma topologia enumeravelmente compacta. Uma continuação natural deste trabalho seria classificar os cardinais $\kappa \leq \mathfrak{c}$ e os grupos abelianos $G$ que admitem uma topologia de grupo cujas potências menores que $\kappa$ são enumeravelmente compactas, mas a $\kappa$-ésima potência não é. 


\section{Referências Bibliográficas}

[1] W.W. Confort, S. Negrepontis, The Theory of Ultrafilters, Springer, Berlin, 1974.

[2] E. K. van Douwen, The product of two countably topological groups, Trans. Amer. Math. Soc. 262 (1980) 417-427

[3] R. Engelking, General Topology, Helderman Verlag (1989)

[4] J. Fraleigh, A first course in abstract algebra, Addison Wesley (2003)

[5] S. Garcia-Ferreira, A.H. Tomita, S. Watson, Countably Compact Groups From A Selective Ultrafilter, Proc. Amer. Math. Soc. 133 (2004) 937-943

[6] K.P. Hart, J. van Mill, A countably compact topological group $H$ such that $H \times H$ is not countably compact, Trans. Amer. Math. Soc. 323 (1991) 811-821

[7] T. Jech, Set Theory, Springer Monographs in Mathematics (2003)

[8] A.H. Tomita, S. Watson, Ultraproducts, p-limits and antichains on the Comfort group order, Topology and its applications 143 (2004) 147-157

[9] A.H. Tomita, Comfort's question on countable compactness, Fudamenta Mathematicae

[10] A.H. Tomita, Countable compactness and finite powers of topological groups without convergent sequences, Topology and its applications 146-147 (2005) 527-538 
[11] A.H. Tomita, Square of countably compact groups without non-trivial convergent sequences, Topology and its applications 153 (2005) 107-117

[12] S. Willard, General Topology, Dover (2004) 